\title{
Stellar parameters of Be stars observed with X-shooter ${ }^{\star}, \star \star$
}

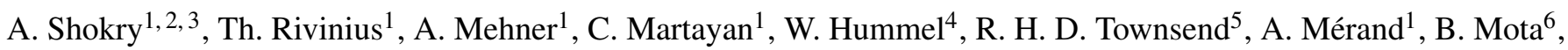
D. M. Faes ${ }^{6}$, M. A. Hamdy ${ }^{2, \star \star \star}$, M. M. Beheary ${ }^{7}$, K. A. K Gadallah ${ }^{7}$, and M. S. Abo-Elazm ${ }^{2}$

${ }^{1}$ ESO - European Organisation for Astronomical Research in the Southern Hemisphere, Casilla 19001, Santiago, Chile e-mail: ahmedsh2911@gmail.com

2 Astronomy Department, National Research Institute of Astronomy and Geophysics (NRIAG), 11421 Helwan, Cairo, Egypt

3 Kottamia Center of Scientific Excellence in Astronomy and Space Science (KCScE, STDF, ASRT), Cairo, Egypt

${ }^{4}$ ESO - European Organisation for Astronomical Research in the Southern Hemisphere, Karl-Schwarzschild-Str. 2, Garchingnwt, Germany

5 Department of Astronomy, University of Wisconsin-Madison, Madison, WI 53706, USA

6 Instituto de Astronomia, Geofísica e Ciências Atmosféricas, Universidade de São Paulo (USP), Rua do Matão 1226, Cidade Universitária, 05508-900 São Paulo, Brazil

7 Astronomy and Meteorology Department, Faculty of Science, Al-Azhar University, Cairo, Egypt

Received 9 July 2017 / Accepted 26 October 2017

\begin{abstract}
Aims. The X-shooter archive of several thousand telluric standard star spectra was skimmed for Be and Be shell stars to derive the stellar fundamental parameters and statistical properties, in particular for the less investigated late-type Be stars and the extension of the Be phenomenon into early A stars.

Methods. An adapted version of the BCD method is used, using the Balmer discontinuity parameters to determine effective temperature and surface gravity. This method is optimally suited for late B stars. The projected rotational velocity was obtained by profile fitting to the $\mathrm{Mg}$ II lines of the targets, and the spectra were inspected visually for the presence of peculiar features such as the infrared $\mathrm{Ca}$ II triplet or the presence of a double Balmer discontinuity. The Balmer line equivalent widths were measured, but they are only useful for determining the pure emission contribution in a subsample of Be stars owing to uncertainties in determining the photospheric contribution.

Results. A total of 78, mostly late-type, Be stars, were identified in the X-shooter telluric standard star archive, out of which 48 had not been reported before. We confirm the general trend that late-type Be stars have more tenuous disks and are less variable than earlytype Be stars. The relatively large number (48) of relatively bright $(V>8.5)$ additional Be stars casts some doubt on the statistics of late-type Be stars; they are more common than currently thought. The Be/B star fraction may not strongly depend on spectral subtype.
\end{abstract}

Key words. circumstellar matter - stars: emission-line, Be - stars: activity

\section{Introduction}

Be stars are non-supergiant B stars that show or have shown $\mathrm{H} \alpha$ emission, as defined by Jaschek et al. (1981). Emission does not only occur in the first members of the Balmer line series, but can affect the continuum and line profiles of other species as well, most often singly ionized metals, such as Fe II. It is generally agreed that, in classical Be stars, this emission is due to the presence of a gaseous Keplerian disk that is concentrated in the equatorial plane. This disk is a decretion disk, i.e., the source of the disk material is the central star, generated by the equatorial flow of stellar material. One of the key factors in creating the disk is supposed to be the very high rotational velocity. In fact, Be stars are known to have higher rotational velocities than normal B-type stars (Catanzaro 2013). For a complete review on the topic, see Porter \& Rivinius (2003) and Rivinius et al. (2013a).

\footnotetext{
* Based on observations made with ESO Telescopes at the La Silla Paranal Observatory under program IDs 60.A-9022, 60.A-9024, 077.D0085, 085.A-0962, 185.D-0056, 091.B-0900, and 093.D-0415.

$\star \star$ Table 6 is only available at the CDS via anonymous ftp to cdsarc.u-strasbg.fr (130.79.128.5) or via http://cdsarc.u-strasbg.fr/viz-bin/qcat?J/A+A/609/A108 $\star \star \star$ Deceased.
}

Classical Be stars are known to vary both in brightness and spectral line appearance with a large range of timescales from years to minutes (Okazaki 1997; Floquet et al. 2002; Kogure \& Leung 2007). While long-term variations are associated with formation and dissipation of the disk (Okazaki 1997), the origin of short-term variability is usually attributed to pulsations within the B star photosphere (Baade 2000; Huat et al. 2009). Photometric studies show that earlier type Be stars are more likely to be variable (e.g., Hubert \& Floquet 1998).

Chojnowski et al. (2015) have demonstrated that Be stars can often be found among stars observed for the purpose of removing telluric absorption in the near-infrared domain because main sequence B stars are among the preferred objects for this task. Inspired by this example, we decided to search for Be stars in a similarly extensive database of telluric standard star observations, namely that taken at the Very Large Telescope (VLT) with the X-shooter instrument.

\section{Observations and data reduction}

All spectra were acquired with the VLT/X-shooter instruments. Most data were taken as telluric standard stars for other observations from the commissioning of X-shooter in 2007 until 

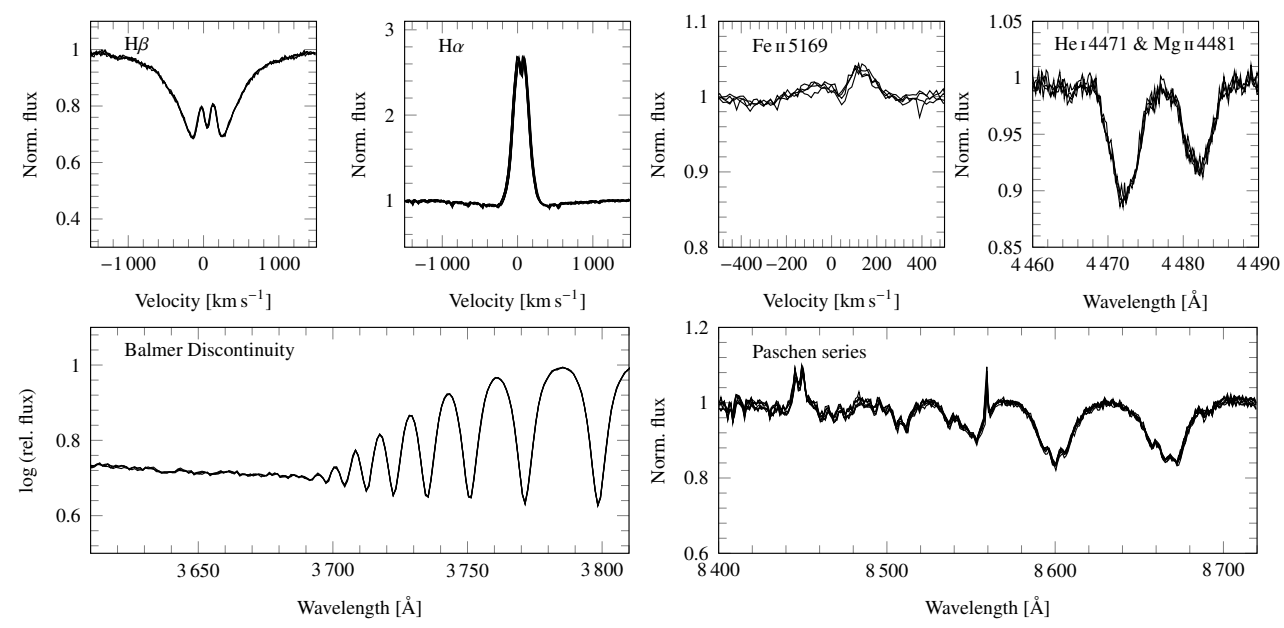

Fig. 1. Spectrum overview plot for HIP 11116. All available spectra are overplotted, giving an indication of the spectral variability. For the remaining 77 active Be stars, see Appendix C.

2015. Once the value of this database for science was realized, additional spectra of stars of interest were acquired in a dedicated observing program in ESO period 93. A few datasets of stars of interest were also taken under a number of different program IDs and were downloaded from the archive as well. The data observed for this work, however, are mainly from program IDs 60.A-9022, 60.A-9024, and 093.D-0415. The meteorological observing conditions, dates, and night-log excerpts are available from the ESO data archive together with the raw data.

$\mathrm{X}$-shooter is a multiwavelength medium-resolution spectrograph mounted at the Cassegrain focus of UT2 of the VLT at ESO Paranal that has a mirror diameter of $8.2 \mathrm{~m}$. The three arms of X-shooter are UVB, covering 300-550 nm; VIS, covering $550-1010 \mathrm{~nm}$; and NIR, covering 1000-2500 nm. The resolution depends on the chosen slit width and ranges from $R=1890$ to 9760 in the UVB, 3180 to 18110 in the VIS, and 3900 to 11490 in NIR arm, respectively (Vernet et al. 2011).

To select the Be stars and other possibly interesting objects from this huge archival sample, first the raw frames in the VIS arm were obtained. A window, containing only the spectral order with the $\mathrm{H} \alpha$ line, was cut from the entire raw frame. From the inter-order space in that window, the local background, effectively the sum of the bias, dark, and scattered light, was estimated and subtracted. The result was integrated into a 1D spectrum, divided by a generic blaze shape obtained from flat-field frames, and then divided by the counts in the continuum. This procedure yields an approximately normalized $\mathrm{H} \alpha$ line profile in the units of pixel vs. flux (i.e., without any wavelength calibration), which is sufficient to judge upon the line shape for the presence of emission and other features of interest.

The resulting more than 10000 profiles of 1334 stars were inspected visually for emission or any other curious appearance, such as binarity or strong profile shape distortions. Of these stars, 1093 were found to be spectroscopically normal BA main sequence objects and 16 were of later spectral type. Of the remaining objects, 89 were spectroscopic binaries of type SB2 (as seen in $\mathrm{H} \alpha$ ) and 48 were found to be supergiants with winds or otherwise not as expected for a single main sequence star. The procedure yielded a number of emission line stars for further inspection, of which two are known as Herbig Be stars (HIP 56379 and HIP 85755), and four are mass-transferring binaries of various types (HIP 33237, HIP 45311, HIP 88615, and HIP 93502). This leaves $78 \mathrm{Be}$ stars identified from the X-shooter data. In addition, among the stars not showing emission four could be identified, with the SIMBAD database, as currently inactive Be stars and hence were added to the sample (HIP 15188, HIP 25950, HIP 108022, and HIP 108975), bringing the number to a total of 82 Be stars. We note that not all stars could be used for every analysis below, so the number of Be stars used for some results might be lower.

The spectra of the Be star sample, identified in the above way, were reduced with the ESO Recipe Flexible Execution Workbench (REFLEX) for X-shooter (Freudling et al. 2013), which is a workflow environment to run ESO VLT pipelines. This workflow provides an easy and interactive way to reduce VLT science data. The steps executed by the ESO X-shooter pipeline (v.2.6.0) include bias subtraction, flat fielding, wavelength and flux calibration, and order merging.

For the flux calibration the master response calibration provided by the ESO archive was used, except for UVB data obtained between 2009 and beginning of 2012. Using the master calibration on these spectra produces an obviously spurious dip in the region immediately blueward of the Balmer discontinuity. This is most likely due to the use of two flat-field lamps in the very blue, where their flux ratio was not entirely stable. To avoid the problem, spectra taken in these years were flux calibrated with a flux standard taken in the same night or not more than a few nights before or after, which was reduced with the same flat-field exposures.

Spectra that had obvious faults, such as bad flux calibration, too little flux, or overexposure in the relevant parts of the continuum, were discarded from the following analysis. This left $10 \mathrm{Be}$ stars without suitable data to determine stellar parameters. For each spectrum, a flux calibrated version and a normalized version, using a global spline fit to continuum regions, were produced for analysis.

In Fig. 1 an overview of the data of the Be star HIP 11116 is shown. Similar plots for the remaining 77 identified Be stars are presented in Appendix C.

\section{Analysis methods}

\subsection{Fundamental parameters via the $B C D$ method}

The primary goal of this work is to obtain fundamental parameters of Be stars. In addition the line broadening $v \sin i$ and Balmer emission equivalent widths (EW) were obtained. 


\subsubsection{Measuring the Balmer discontinuity}

Stellar parameters were determined with a procedure akin to the BCD method, which is named after the main contributors to the method, Barbier, Chalonge, and Divan (see Appendix A of Zorec et al. 2009, for a description, and references in that work for the history of the method). The BCD method uses the height $\left(D_{\star}\right)$ and position $\left(\lambda_{1}\right)$ of the Balmer discontinuity for spectral classification. Some adaptations were necessary, however, since the original BCD method is designed to work on low-resolution photographic spectra with $\Delta \lambda=8 \AA$ at the position of the $\mathrm{BD}$, or about $R=460$.

The height $D_{\star}$ is measured by extrapolating the Balmer and Paschen continua to a wavelength of $370 \mathrm{~nm}$ and taking the flux difference in dex; see the solid and dashed red lines in Fig. 2. For this, both spectra with relative flux calibration and traditionally normalized spectra were used. In the normalized spectrum, a selection was made to chose the points on which the continuum fit would be performed in the flux-calibrated spectrum. All points with a normalized intensity between 0.95 and and 1.05 were selected for this purpose; see the red points in Fig. 2.

- To determine the Paschen continuum, a selection of these points between $\lambda=398$ and $450 \mathrm{~nm}$ was made. A linear regression was computed to the flux-calibrated spectrum in $\log$-log space.

- For the Balmer continuum, and only in cases in which no double Balmer discontinuity (see below) was apparent, the wavelength selection was made between 350 and $368 \mathrm{~nm}$, and then again a linear regression was computed in log-log space.

The difference between the two regressions at $370 \mathrm{~nm}$, which corresponds to 3.568 in log scale, is then taken as $D_{\star}$.

A special problem for Be stars is that the Balmer discontinuity can appear twice: once due to the stellar photosphere and a second time due to the disk, blueward from the photospheric BD. A clearly visible second BD mostly occurs in strong shell and pole-on Be stars. This is due to the lower temperature and pressure broadening in the circumstellar environment (Kaiser 1987; Aidelman et al. 2012). The term shell star here denotes a Be star in which the photosphere is (partly) obscured by the disk. A pole-on star, to the contrary, is a Be star seen at very low inclination. In the shell star, the additional line opacity causes the second $\mathrm{BD}$; in the pole-on star, the second $\mathrm{BD}$ is caused by the line emission, which is typically much higher in pole-on stars, in terms of units of the local continuum, than in Be stars of intermediate inclination. While this does not exclude a secondary $\mathrm{BD}$ in intermediate inclination Be stars, it makes it much harder to detect, as it is typically weaker. Indeed, all stars showing a strong secondary BD are either shell stars, or stars with very narrow photospheric and emission lines. The above method of determining the Balmer continuum would fail here, as it would inevitably focus on the second, circumstellar Balmer discontinuity. For stars that have such a clearly double Balmer discontinuity in their spectrum, the position of points for the linear regression for the Balmer continuum around $370 \mathrm{~nm}$ was defined by hand by selecting the points with the highest flux between Balmer line cores, as demonstrated in the lowermost panel of Fig. 2.

The second parameter measured in the BCD system, $\lambda_{1}$ is the mean spectral position of the Balmer discontinuity, usually given as differential from $370 \mathrm{~nm}$, i.e., $\lambda_{1}=\lambda_{\text {meanBD }}-370$. Following the original BCD convention, $\lambda_{1}$ is given in Ångström in this work. To measure $\lambda_{1}$, the two linear regressions to Paschen and Balmer continuum are averaged to a third linear function
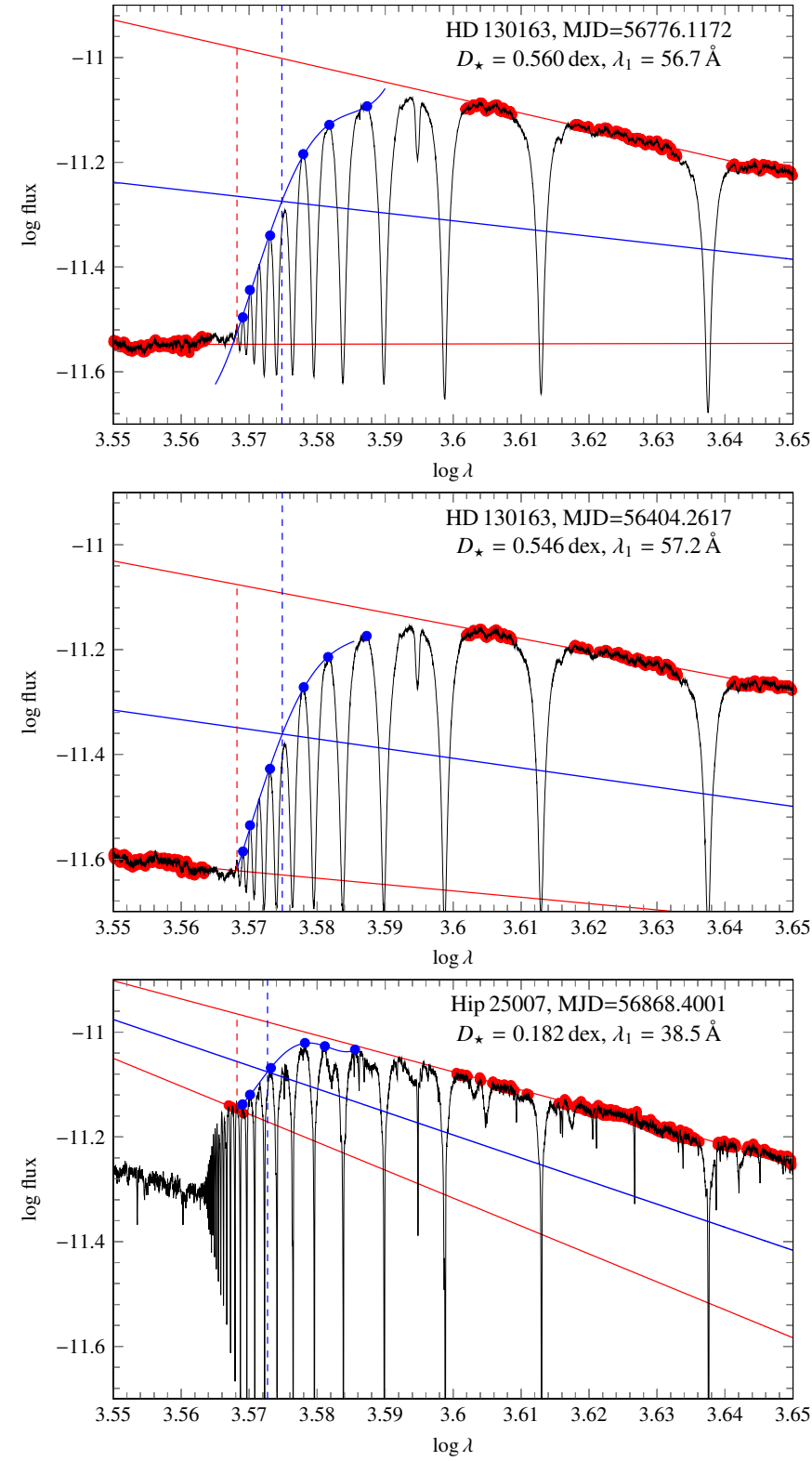

Fig. 2. Two upper panels: spectra for HD 130163 with the most discrepant calibration of the UV flux slope. The steps to determine $D_{\star}$ are indicated in red: selection of continuum points to fit the slopes and the difference between the two fits at $3700 \AA$ as the value of $D_{\star}$. In blue for $\lambda_{1}$ : the midpoints between the continuum fits in red, the upper envelope of the BD. The intersection of this envelope with the midpoints to determine $\lambda_{1}$. Lower panel: for HIP 25007, a clear double BD is present, and the Balmer continuum must be determined by choosing bona fide continuum points manually.

between the two. Next, the midpoints between the Balmer lines, which are local flux maxima, are used for a 4th order polynomial fit to estimate an upper envelope of the flux curve. The wavelength at which this upper envelope intersects the midpoint between Balmer and Paschen continuum is taken to measure $\lambda_{1}$. This position is not independent of the spectral resolution, in particular for lower resolutions as used in the original BCD system.

The principle of measurement is illustrated in Fig. 2. For the non-Be star HD 130163, the results and fit for the two most discrepant observations, in terms of slopes of the Balmer and Paschen continua, are compared. For the strong Be-shell star 
HIP 25007 the procedure in case of a clear double Balmer discontinuity is illustrated.

\subsubsection{Model grid}

To determine stellar parameters, the measurements were compared against a model grid, rather than an existing calibration of the BCD method, since calibrations only exist for low resolution (much lower than the lowest available for X-shooter data). We used the B4 model, which in terms of physics is identical to the BRUCE3 model described in Sect. 3 of Rivinius et al. (2013b), but has been updated computationally to make use of GPU parallel computing. The model creates a surface grid of a rotating star based on the Roche model, and then assigns each point a local value of $\log g$ and $T_{\text {eff }}$ using the Roche model and von Zeipel relations with a traditional value of $\beta=0.25$. The computationally new code then interpolates these precomputed intensity spectra, taking the aspect angle of the line of sight into account. Finally, the numerically updated part integrates the surface grid over the visible surface, a task GPUs were explicitly designed for, into the observed spectrum. This is carried out first for input spectra including all spectral lines, and then for an input grid of spectra with continuum emission only. The resulting spectra can then either be normalized traditionally, i.e., in the same way as the observations, or in a perfect sense, using the computed, true continuum spectrum.

The set of stellar parameters for the input grid is based on Table 6 of Zorec et al. (2009), i.e., computing models based on their parameters for B0 to A1, and for LCs V to III. Any sufficiently dense and even sampling of the $D_{\star}-\lambda_{1}$-plane would have produced the same numerical results for the determined parameters, except it would not have been possible to give an estimate of spectral types. This grid of models was produced for five Xshooter instrumental resolutions in the UVB, which depend on slit width, as mentioned in Sect. 2.

For the spectrophotometric slit of $5^{\prime \prime}$ the resolution is effectively governed by the seeing, for which a somewhat worse than typical value for Paranal observatory of $1.0^{\prime \prime}$ is assumed. The BCD parameters were measured in the model spectra with the same method as the observed data.

To obtain the stellar parameters, the three models forming a triangle that encloses the observed value in the $D_{\star}-\lambda_{1}$-plane were chosen and the barycentric coordinates of the observational $\left(D_{\star}, \lambda_{1}\right)$ pair in this triangle were computed (see Fig. 3). With these coordinates, the physical parameters were interpolated. The spectral type is assigned as one of the most nearby grid points. This is not a spectral classification scheme, which would have to rely on actual standard stars, but is rather only meant as an indication. In a few cases where stars are outside the grid limits, but still close (see Fig. 4), extrapolation was used instead of the barycentric interpolation.

As Be stars are typically fast rotators, the grid was computed for two values of $\omega=\Omega / \Omega_{\text {crit }}$, a slow rotation grid with $\omega=$ 0.25 and a fast rotation grid with $\omega=0.85$. The former was computed for only one value of $v \sin i=20 \mathrm{~km} \mathrm{~s}^{-1}$, i.e., a total of $5 \times 51$ models, the latter for inclinations from a pole-on value of $v \sin i=10 \mathrm{~km} \mathrm{~s}^{-1}$ to the equator in steps of $10 \mathrm{kms}^{-1}$, i.e., a total of $5 \times 651$ models. For the fast rotation grid, at low temperatures and low surface gravities, the equatorial parameters were outside the grid of input spectra and no model spectra were produced for these parameters. The full grids are available at the CDS as Table 6

Most program stars are late type and some are at the very edge of the $D_{\star}-\lambda_{1}$-range covered by the models at $\omega=0.85$.

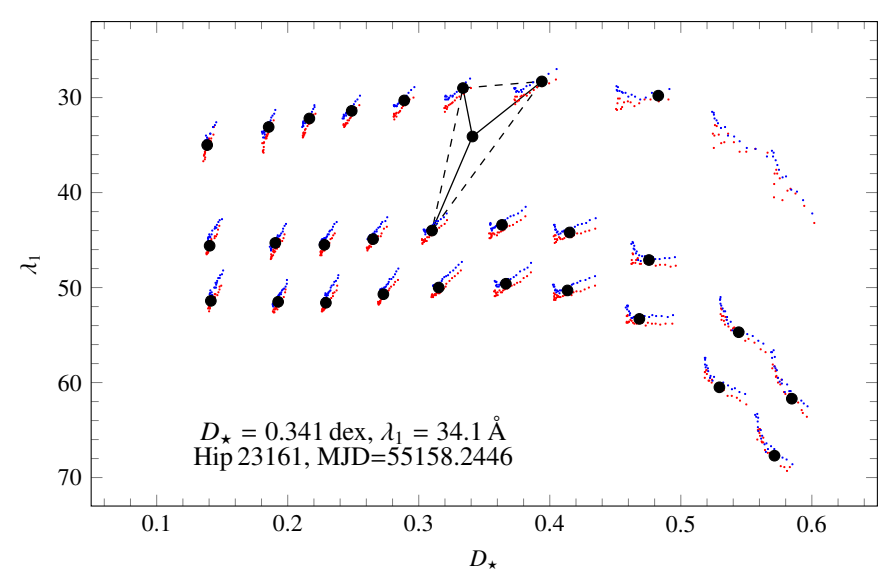

Fig. 3. Interpolation and BCD plane for one star. Shown are all $\left(D_{\star}, \lambda_{1}\right)$ points for all computed $v \sin i$ in the models for $R=3300$ (red) and $R=9100$ (blue). The chosen subgrid to analyze this observation $(R=$ 5100 for $1^{\prime \prime}$ slit width, $v \sin i=150 \mathrm{kms}^{-1}$ ) is shown in black, and the surrounding triangle is indicated by dashed lines, and the barycentric coordinates to obtain the weighted parameter values are indicated by solid lines. The nearest grid point, used to associate a spectral type, represents a B7 III star.

We investigated the influence of rapid rotation on the parameter determination first for a number of test stars only.

\subsubsection{Test cases}

Three non-Be stars with a large number of observations in various slit widths were selected from the archive for the purpose of assessing stability and reliability of the method. These are HIP 72362 (HD 130163, A0 V, V = $6.9 \mathrm{mag}$ ), HIP 98926 (HD 190285, A0 V, $V=7.2 \mathrm{mag}$ ), and HIP 01115 (HD 955, $\mathrm{B} 3 / 5 \mathrm{~V}, V=7.4 \mathrm{mag})$.

In addition, program stars with 10 or more observations were analyzed in a thorough way similar to the three test stars, before applying the method to the bulk of objects with fewer observations. All stars used for tests are given in Table 1.

An absolute flux calibration is not very reliable across the different slit widths, so we only assessed the relative flux calibration. For this, all spectra were normalized to the mean flux in the interval from 404 to $406 \mathrm{~nm}$. The spectra have a large individual scatter, but the averages for the different slit widths are indistinguishable. This indicates that, at the resolutions offered by X-shooter, the differences in resolution do not have much influence on the method employed.

In particular, there is no systematic difference between the observation with the $5^{\prime \prime}$ slit, which does not suffer from any slit loss vs. those with smaller slit widths. This is confirmed by measurements in the model grid, in which the differences between the resolutions turned out to be well below the scatter of the measurements and other systematic errors discussed here (see also Fig. 3). The last two columns of Table 1 give the mean and standard deviation of the measured BCD parameters. Both the height and position of the Balmer discontinuity $D_{\star}$ and $\lambda_{1}$ can be very well measured in X-shooter data. An imperfect flux calibration turns out not to be a problem, since, even if not perfect, it is reasonably stable across the region of interest. In a sense the fitting procedure can be regarded as self-calibrating, and only strong slopes, curvatures, or discontinuities in the flux calibration around $370 \mathrm{~nm}$ would have a strongly detrimental effect on the derived parameters. 
In the next step, and for the non-Be stars only, we investigated how well the $D_{\star}$ and $\lambda_{1}$ values translate into physical parameters. As Table 2 shows, there are systematic effects between the slow rotation and the high rotation model grids. The same BCD parameters analyzed with the slow rotation grid give systematically higher effective temperatures, well outside the statistical scatter. For the effective gravity, the effect is less severe, giving lower $\log g$ for slow rotation, but still within the limit of the statistical error, by which the $3 \sigma$ limit is meant, traditionally employed in astronomy vs. the more conservative $5 \sigma$ limit often found in other fields of physics.

In turn, the differences of the BCD measurements with high rotation, but different inclinations, is negligible. In this context, it does not matter that we do not know the actual rotation of the non-Be test stars. It only matters that we know it for the Be stars, namely that they are rapid rotators, much closer to $85 \%$ than to $25 \%$. It follows that the Be stars must be analyzed with the $85 \%$ grid to avoid the identified systematic errors. However, we do not need to know the inclination or even $v \sin i$ of the Be star with high precision, since this choice does not have a strong effect on the determined BCD parameters.

Combining the errors listed in Tables 1 and 2, one can estimate a typical error of about $50 \mathrm{~K}$ and 0.03 in $\log g$ for a late-type B stars, for which the BCD method has the highest power of distinction (since the BD parameters change steeply at this spectral type), and about $400 \mathrm{~K}$ and 0.05 in $\log g$ for a mid-type B star. Since for most targets only one or two spectra are available, we use these numbers as the typical accuracy.

\subsection{Projected rotational velocity}

When available, $v \sin i$ was taken from the literature; otherwise for each observed spectrum the rotational parameter $v \sin i$ was fitted using synthetic spectra to the Mg II $4481 \AA$ line, which is reasonably strong across the entire range of spectral types investigated in this work, mostly B5 to B9 with a few stars of earlier types only.

For the fit the line profiles were computed with two different sets of underlying model atmospheres. For effective temperatures below $15000 \mathrm{~K}$ ATLAS9 LTE model atmospheres (Kurucz 1969) were used. Above that temperature TLUSTY NLTE atmospheres (Lanz \& Hubeny 2007) were used. As values for $T_{\text {eff }}$ and $\log g$, those obtained by the BCD method were used.

The results of $v \sin i$ for each star are given in Table 3, and Appendix B shows the observed and fitted line profiles for each star.

\subsection{Disk variability and other observations of interest}

Often Be stars show variability in their EW and in the line profiles (Catanzaro 2013). This was checked visually in the spectra, as shown in Appendix $\mathrm{C}$ and flagged in Table 3.

In addition, equivalent widths were measured for the Balmer lines $\mathrm{H} \alpha$ and $\mathrm{H} \beta$, together with the equivalent widths in the models for these stars, see Table 4. The goal to measure the Balmer decrement, i.e., the ratio of emission strength, however, was not achieved with acceptable accuracy. This is because the emission in general is often weak and the Balmer decrement is steep for late-type Be stars. This is seen in the total $\mathrm{H} \beta$ EWs in Table 4 , none of which is negative, i.e., all are still dominated by the photospheric absorption. The values for $\mathrm{H} \beta$ after subtracting the model photospheric EW are dominated by the systematic errors
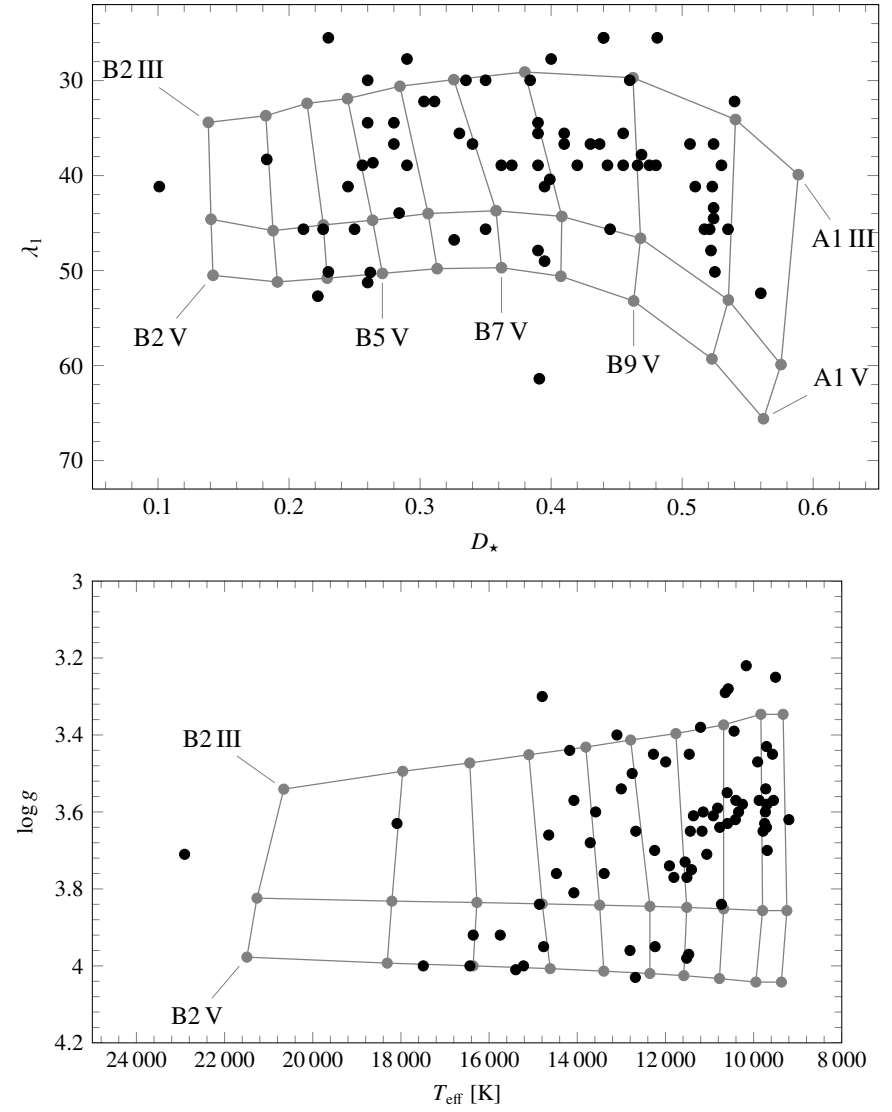

Fig. 4. Distribution of the Be stars in the $D_{\star}-\lambda_{1}$ plane (upper) and $T_{\text {eff }^{-}}$ $\log g$ plane (lower). The model grid points symbolizing the center of the respective spectral types are shown in gray. The bias toward later type Be stars is clearly apparent from the lower panel, with only one star hotter than about $20000 \mathrm{~K}$.

arising from the stellar parameters and the resulting D34 Balmer decrement does not allow us to draw any reliable conclusion.

Some stars show the infrared Ca II triplet in emission. It has been speculated that this is connected to binarity, in particular accretion onto a secondary (Polidan 1976). Koubský et al. (2012) reject this, but acceded that the presence of this line must be due to some peculiarity in the circumstellar environment that is not further specified. The stars for which a clear or possible Ca II triplet emission is observed are also flagged in Table 3.

\section{Results}

In this section, the overall results concerning the sample are reported; for notes and observations on individual stars, see Appendix A.

\subsection{Incidence of Be stars}

To assess the impact of this work on the statistics of Be stars, the selection biases of the sample need to be known and discussed. In fact, the target list is indeed heavily biased toward later type B stars, which is due to the selection policy for telluric standard stars at the VLT. Unless explicitly specified otherwise by the PI of the observations, late-type B and early-type A main sequence stars are preferred. Consequently, the distribution among the luminosity classes V to III is less biased, even though a bias favoring non-giant stars still exists, as not all observers are familiar with the broader definition of main sequence among B stars vs. solar-type telluric stars. Another bias is that 
Table 1. Objects, their derived spectral types, and the number of observations at each slit width to test the method and obtain the statistical scatter and the mean measured $D_{\star}$ and $\lambda_{1}$ values.

\begin{tabular}{|c|c|c|c|c|c|c|c|c|c|}
\hline \multirow[t]{2}{*}{ Star } & \multirow[t]{2}{*}{ Sp Type } & \multicolumn{6}{|c|}{$N$ of observations/slit } & \multirow{2}{*}{$\begin{array}{l}D_{\star} \\
\operatorname{dex}\end{array}$} & \multirow{2}{*}{$\begin{array}{l}\lambda_{1}-3700 \\
\AA\end{array}$} \\
\hline & & $0.5^{\prime \prime}$ & $0.8^{\prime \prime}$ & $1.0^{\prime \prime}$ & $1.3^{\prime \prime}$ & $1.6^{\prime \prime}$ & $5.0^{\prime \prime}$ & & \\
\hline HD 130163 & A1V & - & - & 38 & 5 & 6 & 1 & $0.545 \pm 0.006$ & $63.65 \pm 0.97$ \\
\hline HD 190285 & A1V & 14 & 8 & 15 & 1 & 5 & - & $0.565 \pm 0.013$ & $68.16 \pm 1.1$ \\
\hline HIP 1115 & B3IV & - & 2 & 2 & - & 5 & 2 & $0.253 \pm 0.006$ & $45.65 \pm 2.0$ \\
\hline HIP 32474 & A0IIIe & 1 & - & 7 & - & 2 & - & $0.522 \pm 0.01$ & $44.525 \pm 3.5$ \\
\hline HIP 39483 & B4IIIe & - & 3 & 5 & 1 & 1 & - & $0.281 \pm 0.008$ & $34.44 \pm 1.0$ \\
\hline HIP 52977 & B4IIIe & 7 & - & 5 & 8 & 1 & - & $0.263 \pm 0.017$ & $34.44 \pm 3.04$ \\
\hline HIP 71974 & A0IIIe & 5 & - & 5 & 1 & 2 & - & $0.522 \pm 0.007$ & $47.89 \pm 1.1$ \\
\hline HIP 85138 & B7IIIe & 6 & - & 4 & - & 3 & - & $0.393 \pm 0.011$ & $34.44 \pm 2.4$ \\
\hline HIP 8 & B9IIIe & 5 & - & 16 & 3 & 6 & - & $0.479 \pm 0$ & $38.92 \pm 1.1$ \\
\hline HIP 8 & B9IVe & 1 & 2 & 10 & 2 & 2 & 2 & $0.443 \pm$ & $44.52 \pm 2.07$ \\
\hline HIP 89486 & A0IIIe & 4 & - & 6 & - & 4 & - & $0.512 \pm 0.01$ & $41.16 \pm 0.88$ \\
\hline HIP 94986 & B4IIIe & 8 & 5 & 7 & 1 & 5 & - & $0.278 \pm 0.02$ & $36.68 \pm 3.52$ \\
\hline Hip104508 & B7IIIe & - & 6 & 14 & - & 1 & - & $0.431 \pm 0.014$ & $36.68 \pm 2.95$ \\
\hline
\end{tabular}

Table 2. Stellar parameter for the non-Be stars from Table 1, derived under various assumptions for the stellar rotation.

\begin{tabular}{lcc|cc|ccc}
\hline \hline Star & $\omega=0.25, v \sin i=20 \mathrm{~km} \mathrm{~s}^{-1}$ & \multicolumn{3}{|c|}{$\omega=0.85, v \sin i=20 \mathrm{~km} \mathrm{~s}^{-1}$} & \multicolumn{3}{|c}{$\omega=0.85$, max converging or measured $v \sin i$} \\
\hline & $T_{\text {eff }}$ & $\log g_{\text {pole }}$ & $T_{\text {eff }}$ & $\log g_{\text {pole }}$ & $v \sin i$ & $T_{\text {eff }}$ & $\log g_{\text {pole }}$ \\
& {$[\mathrm{K}]$} & {$[\mathrm{K}]$} & & {$\left[\mathrm{km} \mathrm{s}^{-1}\right]$} & {$[\mathrm{K}]$} & \\
HD 130163 & $9763 \pm 64$ & $3.76 \pm 0.03$ & $9481 \pm 63$ & $3.89 \pm 0.03$ & 120 & $9592 \pm 54$ & $3.86 \pm 0.03$ \\
HD 190285 & \multicolumn{2}{|c|}{ outside of grid } & \multicolumn{2}{|c|}{ outside of grid } & 130 & $9358 \pm 32$ & $3.94 \pm 0.02$ \\
HIP 1115 & $15296 \pm 324$ & $3.78 \pm 0.05$ & $14970 \pm 349$ & $3.91 \pm 0.05$ & 150 & $15119 \pm 358$ & $3.92 \pm 0.05$ \\
\hline
\end{tabular}

Be stars are known to be unsuited as telluric standards, hence there is a bias against known Be stars. Two of those biases can be well seen from Table 5, in that there are generally fewer earlytype stars and, among these, almost no Be stars even if the observed Be star incidence is highest among the early-type B stars (Zorec \& Briot 1997).

Some care is needed to interpret Table 5, however. The spectral types for the non-Be stars were taken from SIMBAD, i.e., they are collected from a large number of inhomogeneous sources. For instance, the original definition of the MK spectral classification scheme did not include standard stars for all subtypes, in particular B4, B6, and B7 were missing and not classified in that scheme at all, while already early the intermediate type of B0.5 was included (see, e.g., Table 1 in Ardeberg 1979). The system has evolved since and more intermediate subtypes as well as standard stars for the missing integer subtypes have been proposed. Nevertheless, the first line of our Table 5 shows a lack of B4, B6, and B7 for exactly this reason. Also, for the purpose of the Table, all intermediate classifications have been rounded to the next earlier integer subtype.

Photometric or spectrophotometric classification systems, such as BCD, on the other hand, define B0 to B9 much more linearly, and also without intermediate types. Therefore, to avoid systematic effects arising from these inconsistencies as much as possible, the sample is grouped into early-, mid-, and latetype stars, which we define as B0-B2, B3-B6, and B7-A1. This amounts to 3 Be stars out of 99 early B stars, 21 out of 313 mid B stars, and 56 out of 681 late B stars.

This lack of early-type Be stars in the sample is because known Be stars have been avoided. The detection probability for an earlier type Be star is much higher, as discussed by, e.g., Zorec et al. (2007). The reason for this bias can be seen from
Appendix C; almost all newly discovered Be stars have weak $\mathrm{H} \alpha$ emission and often no $\mathrm{H} \beta$ at all. Since the traditional spectral classification wavelength range does not include either, but only $\mathrm{H} \gamma$ and bluer Balmer lines, the Be nature is easily missed for stars with steep Balmer decrement.

Figure 3 of Zorec et al. (2007) suggests that the statistics of Be stars is sufficiently complete for spectral types as late as about B6, but becomes increasingly incomplete for B8 and later subtypes. Updating the numbers of Zorec et al. (2007) with our findings does not entirely restore the suggested trend in their Fig. 3, but given that our search was not designed to achieve completeness, it certainly strengthens the suggestion of Zorec et al. (2007) that the probability of a B star to become a Be during its life star does not (strongly) depend on spectral subtype.

\subsection{Stellar parameters}

The values of $D_{\star}$ and $\lambda_{1}$, measured by the method described above, and the stellar parameters $T_{\text {eff }}$ and $\log g$ obtained from these values are given in Table 3 and plotted in the upper panel of Fig. 4. Comparing this to Fig. 1 of Zorec et al. (2005) the values of $\lambda_{1}$ show a systematic offset. This is because of the much higher resolution of X-shooter data vs. nominal BCD method data. At the nominal resolution for the BCD method, the value of $\lambda_{1}$ is strongly affected by the convolution of the stellar spectrum with the instrumental resolution. This is why the original BCD method puts strong emphasis on using spectra at a given resolution of $\Delta \lambda=8 \AA$ at the $\mathrm{BD}$ and the published BCD calibrations cannot be used for X-shooter data. On the other hand, the $\mathrm{X}$-shooter instrumental resolution is so high that its contribution to the BCD parameters is negligible, and our mapping of 
A. Shokry et al.: Stellar parameters of Be stars observed with X-shooter

Table 3. Program stars with the number of valid observations, whether $\mathrm{H} \alpha$ variability, a single, or double $\mathrm{BD}$; the Ca II IR triplet is observed; the measured $D_{\star}$ and $\lambda_{1}$, with mean values in case of more than one observation; and stellar parameters.

\begin{tabular}{|c|c|c|c|c|c|c|c|c|c|c|}
\hline Star & $\begin{array}{l}\text { \# of obs. } \\
\mathrm{U} / \mathrm{V} / \mathrm{N}\end{array}$ & $\begin{array}{l}\text { Sp } \\
\text { Type }\end{array}$ & $\begin{array}{l}\mathrm{H} \alpha \\
\text { var? }\end{array}$ & $\begin{array}{l}\text { Double } \\
\text { BD? }\end{array}$ & $\begin{array}{l}\text { IR Ca II } \\
\text { emiss.? }\end{array}$ & $\begin{array}{l}D_{\star} \\
{[\operatorname{dex}]}\end{array}$ & $\begin{array}{l}\lambda_{1}-3700 \\
{[\AA]}\end{array}$ & $\begin{array}{l}T_{\text {eff }} \\
{[\mathrm{K}]}\end{array}$ & $\begin{array}{l}\log g_{\mathrm{pol}} \\
{[\mathrm{dex}]}\end{array}$ & $\begin{array}{l}v \sin i \\
{\left[\mathrm{~km} \mathrm{~s}^{-1}\right]}\end{array}$ \\
\hline HIP 11116 & $4 / 4 / 4$ & B8III & $\mathrm{N}$ & $\mathrm{N}$ & $\mathrm{N}$ & 0.35 & 29.97 & 11994 & 3.47 & 190 \\
\hline HIP 15188 & $1 / 1 / 1$ & B3: & - & - & $\mathrm{N}$ & - & - & - & - & - \\
\hline HIP 23161 & $2 / 2 / 2$ & B7III & - & $\mathrm{N}$ & $\mathrm{N}$ & 0.335 & 29.97 & 12272 & 3.45 & 150 \\
\hline HIP 24475 & $3 / 4 / 3$ & B9II & $\mathrm{Y}$ & $\mathrm{N}$ & $\mathrm{N}$ & 0.44 & 25.50 & 10643 & 3.29 & 220 \\
\hline HIP 25007 & $6 / 6 / 6$ & B3IV & Y & Y & $\mathrm{N}$ & 0.183 & 38.30 & 18089 & 3.63 & - \\
\hline HIP 25690 & $4 / 4 / 4$ & A0IV & - & $\mathrm{N}$ & $\mathrm{N}$ & 0.525 & 50.14 & 9691 & 3.70 & 90 \\
\hline HIP 25950 & $1 / 2 / 0$ & B7: & $\mathrm{N}$ & - & $\mathrm{N}$ & - & - & - & - & - \\
\hline HIP 26368 & $2 / 2 / 2$ & A1III & $\mathrm{N}$ & $\mathrm{N}$ & $\mathrm{Y}$ & 0.535 & 45.65 & 9550 & 3.57 & 190 \\
\hline HIP 26964 & $2 / 2 / 1$ & B4V & - & $Y$ & $\mathrm{~N}$ & 0.262 & 50.2 & 15220 & 4.00 & 200 \\
\hline HIP 28561 & $3 / 3 / 3$ & B8IV & $\mathrm{Y}$ & $\mathrm{Y}$ & $Y$ & 0.399 & 40.4 & 11554 & 3.73 & 70 \\
\hline HIP 29635 & $8 / 8 / 9$ & B8IV & $\mathrm{N}$ & $\mathrm{N}$ & $\mathrm{Y}$ & 0.395 & 41.16 & 11409 & 3.75 & 230 \\
\hline HIP 31362 & $1 / 1 / 1$ & B8IV & - & $\mathrm{N}$ & $\mathrm{N}$ & 0.443 & 38.92 & 10769 & 3.64 & 270 \\
\hline HIP 32474 & $10 / 14 / 16$ & A0III & $\mathrm{Y}$ & $\mathrm{N}$ & $\mathrm{N}$ & 0.524 & 44.52 & 9733 & 3.60 & 120 \\
\hline HIP 33509 & $1 / 1 / 1$ & B4V & - & Y & $\mathrm{N}$ & 0.226 & 45.65 & 15747 & 3.92 & 160 \\
\hline HIP 34144 & $1 / 1 / 1$ & B8III & - & $\mathrm{N}$ & $\mathrm{N}$ & 0.4 & 27.73 & 11204 & 3.38 & 200 \\
\hline HIP 36009 & $1 / 1 / 1$ & B4V & - & $\mathrm{N}$ & $\mathrm{N}$ & 0.23 & 50.14 & 16435 & 4.00 & 40 \\
\hline HIP 37007 & $6 / 6 / 6$ & B7IV & $\mathrm{N}$ & $\mathrm{N}$ & $\mathrm{Y}$ & 0.34 & 36.68 & 12245 & 3.70 & 230 \\
\hline HIP 39183 & $2 / 2 / 2$ & A0III & $\mathrm{Y}$ & $\mathrm{N}$ & : & 0.481 & 25.50 & 10167 & 3.22 & 190 \\
\hline HIP 39483 & $10 / 11 / 11$ & B6IV & Y & $\mathrm{N}$ & $\dot{N}$ & 0.28 & 34.44 & 13583 & 3.60 & 130 \\
\hline HIP 39595 & $1 / 1 / 1$ & B9IV & - & $\mathrm{N}$ & Y & 0.455 & 38.92 & 10592 & 3.63 & 240 \\
\hline HIP 41085 & $1 / 1 / 1$ & B5IV & - & Y & $\mathrm{N}$ & 0.245 & 41.16 & 14860 & 3.84 & 250 \\
\hline HIP 41268 & $2 / 2 / 2$ & B8III & - & $\mathrm{Y}$ & $\mathrm{N}$ & 0.384 & 29.97 & 11460 & 3.45 & 240 \\
\hline HIP 42060 & $1 / 1 / 1$ & B7V & - & $\mathrm{N}$ & $\mathrm{N}$ & 0.35 & 45.65 & 12235 & 3.95 & 250 \\
\hline HIP 43073 & $1 / 1 / 1$ & B9IV & - & $\mathrm{N}$ & $\mathrm{N}$ & 0.466 & 38.92 & 10413 & 3.62 & 250 \\
\hline HIP 43114 & $1 / 1 / 0$ & B5IV: & - & $\mathrm{N}$ & $\mathrm{N}$ & - & - & - & $\begin{array}{l}0.02 \\
-\end{array}$ & - \\
\hline HIP 44423 & $2 / 3 / 3$ & B7IV & $\mathrm{N}$ & $\mathrm{N}$ & $\mathrm{N}$ & 0.362 & 38.92 & 11909 & 3.74 & 160 \\
\hline HIP 46329 & $2 / 2 / 2$ & B5IV & $\mathrm{Y}$ & $\mathrm{N}$ & $\mathrm{N}$ & 0.25 & 45.65 & 14763 & 3.95 & 160 \\
\hline HIP 47868 & $1 / 1 / 1$ & B0III: & - & $\mathrm{N}$ & $\mathrm{N}$ & 0.067 & 23.70 & - & - & - \\
\hline HIP 47962 & $1 / 3 / 3$ & AOIV & $\mathrm{N}$ & $\mathrm{N}$ & $\mathrm{N}$ & 0.517 & 45.65 & 9786 & 3.65 & 220 \\
\hline HIP 48582 & $4 / 6 / 6$ & B5IV & Y & Y & Y & 0.26 & 51.26 & 15396 & 4.01 & - \\
\hline HIP 48943 & $2 / 5 / 2$ & B5IV & $\mathrm{N}$ & $\mathrm{Y}$ & $\mathrm{Y}$ & 0.284 & 43.95 & 14077 & 3.81 & 190 \\
\hline HIP 51444 & $1 / 2 / 2$ & B5III & Y & $\mathrm{N}$ & $\mathrm{N}$ & 0.23 & 25.50 & 14795 & 3.30 & 250 \\
\hline HIP 51491 & $3 / 3 / 4$ & B9III & $\mathrm{Y}$ & $\mathrm{N}$ & Y & 0.475 & 38.92 & 10341 & 3.60 & 230 \\
\hline HIP 51546 & $2 / 2 / 2$ & A0V: & - & $\mathrm{N}$ & $\mathrm{N}$ & - & - & - & - & 210 \\
\hline HIP 52977 & $21 / 21 / 21$ & B6III & $\mathrm{Y}$ & $\mathrm{N}$ & $\mathrm{N}$ & 0.26 & 34.44 & 14076 & 3.57 & 200 \\
\hline HIP 56393 & $1 / 1 / 1$ & A1IV & - & $\mathrm{N}$ & : & 0.56 & 52.39 & 9200 & 3.62 & 270 \\
\hline HIP 57861 & $4 / 6 / 6$ & B6IV & Y & $\mathrm{N}$ & $\dot{N}$ & 0.29 & 38.92 & 13390 & 3.76 & 270 \\
\hline HIP 59970 & $2 / 2 / 2$ & A1IV-III & - & $\mathrm{N}$ & : & 0.574 & 48.45 & - & - & - \\
\hline HIP 64501 & $2 / 2 / 2$ & B8IV & - & $\mathrm{N}$ & $\mathrm{N}$ & 0.39 & 38.92 & 11512 & 3.77 & - \\
\hline HIP 64867 & $5 / 6 / 6$ & A0III & $\mathrm{N}$ & $\mathrm{N}$ & $\mathrm{N}$ & 0.524 & 36.68 & 9708 & 3.43 & 170 \\
\hline HIP 66339 & $1 / 1 / 1$ & B3V & - & $\mathrm{Y}$ & $\mathrm{N}$ & 0.222 & 52.7 & 17493 & 4.00 & 210 \\
\hline HIP 66351 & $2 / 2 / 2$ & B9III & - & $\mathrm{N}$ & $\mathrm{N}$ & 0.469 & 37.80 & 10404 & 3.57 & 140 \\
\hline
\end{tabular}

Notes. For the double BD and Ca II flags "Y" and "N" are clear statements, ":" means uncertain, and "_" mean no suitable spectra were available. Newly identified Be stars are indicated in bold face. In case of $\mathrm{H} \alpha$ variability, "-" means that all spectra, even if more than one, were taken on the same night.

$T_{\text {eff }}$ and $\log g$ onto $D_{\star}$ and $\lambda_{1}$ is valid for all medium- to highresolution data.

Table 5 also confirms the finding of Zorec et al. (2005) that among the later type Be stars the higher luminosity classes are more common, i.e., later type Be stars are more likely found in the second half of their main sequence life. This is in agreement with the idea that the Be phase is a consequence of rotational evolution during the main sequence (e.g., Granada et al. 2013). In that hypothesis, a Be star with moderate rotation at the ZAMS, owing to the internal evolution and angular momentum transport from core to surface, at some point approaches critical rotation at the surface as the star ages. To prevent the surface rotation from going above the critical threshhold, angular momentum must be transported away. The means of this transport then is the circumstellar decretion disk (Krtička et al. 2011).

\subsection{Disk properties as a function of spectral type}

Although it was not possible to determine the Balmer decrement for our sample stars (see Table 4 for measurements of the equivalent widths), this still provides information. Because the emission is weak and the Balmer decrement too steep, the disks found by $\mathrm{X}$-shooter are too tenuous to allow a reliable measurement of the Balmer decrement. This is in agreement with 
Table 3. continued.

\begin{tabular}{|c|c|c|c|c|c|c|c|c|c|c|}
\hline Star & $\begin{array}{l}\text { \# of obs. } \\
\mathrm{U} / \mathrm{V} / \mathrm{N}\end{array}$ & $\begin{array}{l}\text { Sp } \\
\text { Type }\end{array}$ & $\begin{array}{l}\mathrm{H} \alpha \\
\text { var? }\end{array}$ & $\begin{array}{l}\text { Double } \\
\text { BD? }\end{array}$ & $\begin{array}{l}\text { IR Ca II } \\
\text { emiss.? }\end{array}$ & $\begin{array}{l}D_{\star} \\
{[\operatorname{dex}]}\end{array}$ & $\begin{array}{l}\lambda_{1}-3700 \\
{[\AA]}\end{array}$ & $\begin{array}{l}T_{\text {eff }} \\
{[\mathrm{K}]}\end{array}$ & $\begin{array}{l}\log g_{\mathrm{pol}} \\
{[\mathrm{dex}]}\end{array}$ & $\begin{array}{l}v \sin i \\
{\left[\mathrm{~km} \mathrm{~s}^{-1}\right]}\end{array}$ \\
\hline HIP 68100 & $2 / 2 / 2$ & B6V & - & $\mathrm{N}$ & $\mathrm{N}$ & 0.326 & 46.77 & 12804 & 3.96 & 100 \\
\hline HIP 69429 & $4 / 6 / 6$ & A0IV & $\mathrm{N}$ & $\mathrm{N}$ & $\mathrm{N}$ & 0.521 & 45.65 & 9749 & 3.63 & 190 \\
\hline HIP 71668 & $3 / 3 / 3$ & B2V: & $\mathrm{N}$ & $\mathrm{N}$ & $\mathrm{N}$ & - & - & - & - & - \\
\hline HIP 71974 & $12 / 141 / 4$ & AOIV & $\mathrm{N}$ & $\mathrm{N}$ & $\mathrm{N}$ & 0.522 & 47.89 & 9709 & 3.64 & 160 \\
\hline HIP 78375 & $1 / 1 / 1$ & B9: & $\mathrm{N}$ & $\mathrm{N}$ & $:$ & - & - & - & - & - \\
\hline HIP 80577 & $3 / 3 / 3$ & B8IV & $\mathrm{Y}$ & $\mathrm{N}$ & $\mathrm{N}$ & 0.39 & 47.89 & 11472 & 3.97 & 100 \\
\hline HIP 80820 & $3 / 3 / 3$ & B9III & Y & $\mathrm{N}$ & $\mathrm{N}$ & 0.44 & 25.50 & 10576 & 3.28 & 150 \\
\hline HIP 81321 & $0 / 2 / 2$ & A0V: & - & - & $\mathrm{N}$ & - & - & - & - & - \\
\hline HIP 82874 & $1 / 1 / 0$ & B7III & - & $\mathrm{N}$ & $\mathrm{N}$ & 0.311 & 32.20 & 12755 & 3.50 & 240 \\
\hline HIP 83278 & $3 / 3 / 3$ & B9IV & $\mathrm{N}$ & $\mathrm{N}$ & $\mathrm{N}$ & 0.437 & 36.68 & 10816 & 3.59 & 230 \\
\hline HIP 84184 & $3 / 3 / 3$ & B8IV & $\mathrm{Y}$ & $\mathrm{Y}$ & $\mathrm{N}$ & 0.37 & 38.92 & 11808 & 3.77 & 270 \\
\hline HIP 85138 & $14 / 15 / 15$ & B8IV & Y & $\mathrm{N}$ & $\mathrm{N}$ & 0.39 & 34.44 & 11365 & 3.61 & 180 \\
\hline HIP 85195 & $28 / 29 / 29$ & B9III & $\mathrm{Y}$ & $\mathrm{N}$ & $\mathrm{N}$ & 0.48 & 38.92 & 10253 & 3.58 & 250 \\
\hline HIP 85566 & $3 / 3 / 3$ & A1III & $\mathrm{N}$ & $\mathrm{N}$ & $:$ & 0.53 & 38.92 & 9572 & 3.45 & 240 \\
\hline HIP 87032 & $3 / 3 / 3$ & B8V: & $\mathrm{N}$ & $\mathrm{N}$ & $\mathrm{Y}$ & - & - & - & - & 300 \\
\hline HIP 87698 & $1 / 2 / 2$ & A0III & $\mathrm{N}$ & $\mathrm{N}$ & $\mathrm{N}$ & 0.523 & 41.16 & 9725 & 3.54 & 210 \\
\hline HIP 88172 & $2 / 5 / 3$ & B8IV & $\mathrm{N}$ & $\mathrm{N}$ & $\mathrm{N}$ & 0.39 & 35.56 & 11436 & 3.65 & 180 \\
\hline HIP 88374 & $21 / 25 / 25$ & B9IV & Y & $\mathrm{N}$ & $\mathrm{N}$ & 0.445 & 45.65 & 10730 & 3.84 & 140 \\
\hline HIP 89486 & $14 / 14 / 15$ & A0III & Y & $\mathrm{N}$ & $\mathrm{N}$ & 0.51 & 41.16 & 9874 & 3.57 & 180 \\
\hline HIP 89500 & $5 / 5 / 5$ & B9III & $\mathrm{N}$ & $\mathrm{N}$ & $\mathrm{N}$ & 0.46 & 29.97 & 10443 & 3.39 & 240 \\
\hline HIP 90096 & $4 / 5 / 5$ & B8IV & $\mathrm{N}$ & $\mathrm{N}$ & $\mathrm{N}$ & 0.41 & 36.68 & 11170 & 3.65 & 200 \\
\hline HIP 90509 & $2 / 2 / 2$ & B8IV & - & $\mathrm{N}$ & : & 0.42 & 38.92 & 11062 & 3.71 & 250 \\
\hline HIP 91460 & $3 / 3 / 3$ & A1III & $\mathrm{N}$ & $\mathrm{N}$ & $\mathrm{N}$ & 0.54 & 32.20 & 9503 & 3.25 & 150 \\
\hline HIP 91975 & $2 / 3 / 3$ & B9III & $\mathrm{Y}$ & $\mathrm{N}$ & $:$ & 0.455 & 35.56 & 10600 & 3.55 & 250 \\
\hline HIP 92038 & $3 / 3 / 3$ & B7III & $\mathrm{Y}$ & $\mathrm{Y}$ & $\mathrm{Y}$ & 0.303 & 32.20 & 13002 & 3.54 & 170 \\
\hline HIP 93993 & $3 / 3 / 3$ & A0III & $\mathrm{N}$ & $\mathrm{N}$ & $\mathrm{N}$ & 0.524 & 43.40 & 9694 & 3.58 & 10 \\
\hline HIP 94770 & $2 / 2 / 2$ & B8IV & - & $\mathrm{N}$ & $\mathrm{Y}$ & 0.41 & 35.56 & 11145 & 3.60 & 260 \\
\hline HIP 94859 & $1 / 3 / 3$ & B7III & Y & $\mathrm{N}$ & : & 0.33 & 35.56 & 12673 & 3.65 & 240 \\
\hline HIP 94986 & $24 / 36 / 36$ & B6III & $\mathrm{Y}$ & $\mathrm{N}$ & $\mathrm{N}$ & 0.28 & 36.68 & 13706 & 3.68 & 120 \\
\hline HIP 95109 & $1 / 2 / 2$ & B8V & $\mathrm{N}$ & $\mathrm{N}$ & $\mathrm{N}$ & 0.395 & 49.01 & 11518 & 3.98 & 260 \\
\hline HIP 96453 & $1 / 3 / 3$ & B6III & $\mathrm{Y}$ & $\mathrm{N}$ & $\mathrm{N}$ & 0.26 & 29.97 & 14172 & 3.44 & 230 \\
\hline HIP 99457 & $7 / 9 / 3$ & B1IV & $\mathrm{Y}$ & $\mathrm{N}$ & $\mathrm{N}$ & 0.101 & 41.16 & 22907 & 3.71 & - \\
\hline HIP 100664 & $2 / 3 / 3$ & A0III & $\mathrm{N}$ & $\mathrm{N}$ & $\mathrm{N}$ & 0.506 & 36.68 & 9908 & 3.47 & 190 \\
\hline HIP 104508 & $18 / 24 / 24$ & B9IV & $\mathrm{Y}$ & $\mathrm{N}$ & $\mathrm{N}$ & 0.43 & 36.68 & 10915 & 3.61 & 270 \\
\hline HIP 108022 & $2 / 5 / 5$ & B6III & $\mathrm{N}$ & $\mathrm{N}$ & $\mathrm{N}$ & 0.29 & 27.73 & 13100 & 3.40 & 110 \\
\hline HIP 108402 & $5 / 5 / 5$ & B7V & $\mathrm{N}$ & $\mathrm{Y}$ & $\mathrm{Y}$ & 0.391 & 61.39 & 12682 & 4.03 & 250 \\
\hline HIP 108597 & $3 / 3 / 3$ & B5IV & $\mathrm{N}$ & Y & $\mathrm{Y}$ & 0.256 & 38.92 & 14473 & 3.76 & - \\
\hline HIP 108975 & $2 / 2 / 2$ & B4IV & $\mathrm{N}$ & $\mathrm{N}$ & $\mathrm{N}$ & 0.264 & 38.65 & 14648 & 3.66 & - \\
\hline
\end{tabular}

Vieira et al. (2017), who found that disks of late-type Be stars are less dense than those of early-type stars. As mentioned above, there is also a general agreement that late-type Be stars show less variability than early-type stars. This is again confirmed by the numbers shown in Table 5, where two-thirds of early- and mid-type Be stars are found to be variable, but only about one-third are variable among the late subtypes.

\subsection{Infrared Ca II triplet}

We clearly detected the IR Ca II triplet in the emission of 13 stars. This is in agreement with the reported number of about $20 \%$ of Be stars showing this feature (e.g., Koubský et al. 2012). In absorption and possibly emission the IR Ca II triplet is seen in another 8 stars.

There is no obvious correlation of the presence of the IR Ca II triplet, in either emission or absorption, with spectral type of the Be star. Although our sample (heavily biased toward later type Be stars) does not include stars with Ca II triplet emission earlier than B5, a literature search does reveal such stars (Polidan \& Peters 1976; Polidan 1976; Briot 1981; Koubský et al. 2012).

The emission morphology closely resembles that of the O I 8446 line, which is supposed to trace the hydrogen $\operatorname{Ly} \beta$ formation region, since its upper level is excited by fluorescence from this transition (Mathew et al. 2012). However, while the O I 8446 can be clearly linked to processes originating in the Be star, this is not the case for the IR Ca II triplet.

The emission strength of the Ca II triplet can vary without similar changes taking place in O I 8446 or the Balmer lines. This emission strength can even be transient without a major change in the Balmer line emission properties; for example, the Ca II triplet was not detected in $\gamma$ Cas by Briot (1981), but was reported to be present by Koubský et al. (2012) to be anticorrelated with the Balmer emission for the same star.

This suggests that while the formation region is the same as for the other lines, i.e., the disk around the Be star, the excitation process does not originate in the same source as for the other spectral lines formed in the disk. Koubský et al. (2012) investigated the correlation of the Ca II triplet with binarity and 
A. Shokry et al.: Stellar parameters of Be stars observed with X-shooter

Table 4. Equivalent widths of $\mathrm{H} \alpha$ and $\mathrm{H} \beta$ for the program stars with clearly measurable emission.

\begin{tabular}{cccccccc}
\hline \hline Star & $\begin{array}{c}T_{\text {eff }} \\
{[\mathrm{K}]}\end{array}$ & $\begin{array}{c}\mathrm{H} \alpha \\
\text { (tot) }\end{array}$ & $\begin{array}{c}\mathrm{phot}) \\
\text { (emi) }\end{array}$ & $\begin{array}{c}\mathrm{H} \beta \\
\text { (tot) }\end{array}$ & $\begin{array}{c}\text { phot) } \\
\text { (emi) }\end{array}$ \\
\hline HIP 11116 & 12240 & -7.94 & 4.20 & -12.15 & 5.23 & 7.24 & -2.00 \\
HIP 26368 & 9874 & 5.39 & 6.83 & -1.435 & 10.8 & 12.1 & -1.28 \\
HIP 29635 & 11822 & -1.69 & 5.39 & -7.085 & 7.76 & 9.55 & -1.78 \\
HIP 33509 & 17064 & -16.4 & 3.57 & -20.06 & 3.31 & 6.40 & -3.08 \\
HIP 37007 & 15093 & -5.95 & 4.53 & -10.48 & 6.06 & 7.57 & -1.50 \\
HIP 39183 & 11231 & 0.65 & 5.89 & -5.235 & 7.68 & 8.51 & -0.82 \\
HIP 39595 & 12234 & -1.65 & 5.68 & -7.337 & 9.12 & 10.1 & -1.03 \\
HIP 46329 & 14599 & -8.91 & 4.27 & -13.18 & 5.28 & 6.85 & -1.56 \\
HIP 51491 & 11453 & 4.44 & 5.94 & -1.493 & 8.39 & 10.5 & -2.12 \\
HIP 80577 & 11697 & 0.43 & 5.94 & -5.503 & 9.07 & 10.1 & -1.09 \\
HIP 82874 & 14099 & -6.90 & 3.93 & -10.83 & 5.13 & 6.75 & -1.61 \\
HIP 87032 & 11936 & -6.37 & 6.70 & -13.07 & 7.89 & 11.1 & -3.22 \\
HIP 90096 & 13441 & 3.05 & 5.20 & -2.146 & 8.56 & 9.02 & -0.45 \\
HIP 90509 & 13123 & 0.44 & 5.37 & -4.925 & 8.76 & 9.51 & -0.74 \\
HIP 91975 & 11951 & 1.64 & 5.48 & -3.830 & 7.70 & 9.45 & -1.74 \\
HIP 92038 & 15139 & -14.4 & 3.80 & -18.23 & 3.80 & 5.98 & -2.18 \\
HIP 94770 & 12721 & -2.43 & 5.12 & -7.561 & 7.70 & 8.84 & -1.13 \\
HIP 94859 & 15311 & -2.25 & 4.26 & -6.523 & 6.80 & 8.12 & -1.31 \\
HIP 95109 & 11847 & -2.64 & 5.96 & -8.601 & 8.56 & 10.2 & -1.68 \\
HIP 96453 & 14338 & 2.81 & 4.52 & -1.706 & 5.51 & 5.77 & -0.25 \\
HIP 99457 & 23061 & -1.37 & 2.51 & -3.889 & 2.73 & 3.71 & -0.97 \\
\hline
\end{tabular}

Table 5. Statistics of Be stars observed with X-shooter.

\begin{tabular}{|c|c|c|c|c|c|c|c|c|c|c|c|c|c|c|c|}
\hline Bin name & & Early & & $\Sigma$ & & & & & $\Sigma$ & & & Late & & & $\Sigma$ \\
\hline Sp. type & B0 & B1 & B2 & & B3 & B4 & B5 & B6 & & B7 & B8 & B9 & A0 & A1 & \\
\hline \# of all stars & 4 & 10 & 85 & 99 & 129 & 38 & 112 & 34 & 313 & 57 & 211 & 347 & 52 & 14 & 681 \\
\hline \# of Be stars & 1 & 1 & 1 & 3 & 3 & 4 & 7 & 7 & 21 & 9 & 16 & 13 & 13 & 5 & 56 \\
\hline \# of new Be stars & 0 & 0 & 0 & 0 & 0 & 1 & 3 & 5 & 9 & 6 & 10 & 8 & 11 & 4 & 39 \\
\hline LC V & 0 & 0 & 1 & 1 & 1 & 3 & 0 & 1 & 5 & 2 & 2 & 0 & 1 & 0 & 5 \\
\hline LC IV & 0 & 1 & 0 & 1 & 1 & 1 & 5 & 2 & 9 & 2 & 11 & 5 & 5 & 2 & 25 \\
\hline LC III & 1 & 0 & 0 & 1 & 0 & 0 & 1 & 4 & 5 & 4 & 3 & 6 & 7 & 3 & 23 \\
\hline Ca II emi/abs & 0 & 0 & 0 & 0 & 0 & 0 & 3 & 0 & 3 & 4 & 5 & 4 & 1 & 4 & 18 \\
\hline Variability detectable? & 0 & 1 & 1 & 2 & 1 & 1 & 5 & 6 & 13 & 6 & 11 & 10 & 10 & 3 & 40 \\
\hline $\mathrm{H} \alpha$ variable & 0 & 1 & 0 & 1 & 1 & 0 & 3 & 5 & 9 & 2 & 4 & 6 & 2 & 0 & 14 \\
\hline
\end{tabular}

conclude that binarity is not the responsible mechanism, but suggest some other, not further specified peculiarity of the circumstellar disk.

For young stellar objects the common presence of the Ca II triplet in emission is suspected to be linked to either magnetic processes, accretion (e.g., Kwan \& Fischer 2011; Moto'oka \& Itoh 2013), or a combination of both. In cataclysmic variables, the formation of the triplet is more specifically traced to external UV irradiation of an optically thin gas (Ivanova et al. 2004). If we combine that with the current understanding of Be stars, which do not show any trace of largescale magnetic fields, this leaves UV photons formed in accretion shocks as the most promising mechanism to power the Ca II triplet. The self re-accretion from the viscous disk is probably not sufficient, as otherwise almost all Be stars should have Ca II emission.

This leaves binarity as a possible explanation. As Koubský et al. (2012) point out, several known binaries do not show Ca II in their data. However, plain binarity is not sufficient; the companion must also accrete to form the UV flux to excite Ca II. Hence binarity remains a possible hypothesis to explain the infrared Ca II triplet emission.

\section{Conclusions}

Searching the X-shooter database of telluric standards, 78 Be stars were detected in emission, of which 48 had not been reported before. The sample is strongly biased toward later type Be stars. In some sense, this is an advantage, because later type Be stars, owing to their lack of variability and often less dense disks, are less well studied than earlier type Be stars. In particular, we were able to confirm, or at least strengthen, the following findings and hypotheses:

- The Galactic Be star fraction decreases less dramatically toward the later spectral (sub-)types than previously known 
numbers suggest. It may even be constant, as proposed by Zorec et al. (2005).

- Late-type Be stars show less variablility of their disks than early-type Be stars.

- Late-type Be stars have less dense disks than early-type Be stars.

- Be stars are more likely to be closer to the TAMS than to the ZAMS.

- The presence of the IR Ca II in emission may be linked to accretion onto a companion, but the emission itself originates from the Be disk proper.

Some of these points either clearly are, or may well be, linked to stellar evolution and its timescales. For instance the lower density of disks around later subtype Be stars could be a natural consequence of their slower evolution, if indeed the disk is the means by which the star stays below critical rotation. The amount of angular momentum to lose over a given time is simply less. The same might explain the lower variability of the later subtypes.

In summary, while late-type Be stars are less well investigated than the earlier type Be stars, it might actually be this lack of "interesting" behavior in them that will enable new insights into the origin and evolution of Be stars.

Acknowledgements. We dedicate this work to the memory of Prof. M. Hamdy. A. Shokry would like to acknowledge the Egyptian Ministry of Higher Education (MoHE) for providing the financial support for his Joint scholarship, as well as the ESO observatory for offering facilities and tools needed through his stay in ESO (Chile), and the Kottamia Center of Scientific Excellence for support. A. Shokry is deeply indebted to Prof. S. Saad for her continued support. R. Townsend acknowledges support from NASA grant NNX12AC72G. D.M.F. acknowledges support from FAPESP grant 2016/16844-1. This research has made use of NASA's Astrophysics Data System Service, as well as of the SIMBAD database, operated at CDS, Strasbourg, France.

\section{References}

Aidelman, Y., Cidale, L. S., Zorec, J., \& Arias, M. L. 2012, A\&A, 544, A64

Andersen, J., \& Nordstrom, B. 1983, A\&AS, 52, 471

Ardeberg, A. 1979, in IAU Colloq. 47: Spectral Classification of the Future, eds M. F. McCarthy, A. G. D. Philip, \& G. V. Coyne, Ricerche Astronomiche, 9, 389

Baade, D. 2000, in IAU Colloq. 175: The Be Phenomenon in Early-Type Stars, eds. M. A. Smith, H. F. Henrichs, \& J. Fabregat, ASP Conf. Ser., 214, 178

Balona, L. A. 1975, MmRAS, 78, 51

Balona, L. A. 1990, MNRAS, 245, 92

Bhatt, H. C., Ashok, N. M., Chandrasekhar, T., \& Goraya, P. S. 1984, A\&AS, 58,685

Briot, D. 1981, A\&A, 103, 1

Briot, D. 1986, A\&A, 163, 67

Buscombe, W. 1970, MNRAS, 148, 79

Carrier, F., Burki, G., \& Burnet, M. 2002, A\&A, 385, 488

Catanzaro, G. 2013, A\&A, 550, A79

Chojnowski, S. D., Whelan, D. G., Wisniewski, J. P., et al. 2015, AJ, 149, 7
Dall, T. H., Foellmi, C., Pritchard, J., et al. 2007, A\&A, 470, 1201 Floquet, M., Neiner, C., Janot-Pacheco, E., et al. 2002, A\&A, 394, 137 Frémat, Y., Zorec, J., Hubert, A.-M., \& Floquet, M. 2005, A\&A, 440, 305 Freudling, W., Romaniello, M., Bramich, D. M., et al. 2013, A\&A, 559, A96 Ghosh, K. K., Apparao, K. M. V., \& Pukalenthi, S. 1999, A\&AS, 134, 359 Goraya, P. S., \& Tur, N. S. 1988, AJ, 96, 346

Granada, A., Ekström, S., Georgy, C., et al. 2013, A\&A, 553, A25

Gray, R. O., \& Corbally, C. J. 2002, AJ, 124, 989

Halbedel, E. M. 1996, PASP, 108, 833

Harrington, D. M., \& Kuhn, J. R. 2009, ApJS, 180, 138

Henize, K. G. 1976, ApJS, 30, 491

Hiltner, W. A., Garrison, R. F., \& Schild, R. E. 1969, ApJ, 157, 313

Huat, A.-L., Hubert, A.-M., Baudin, F., et al. 2009, A\&A, 506, 95

Hubert, A. M., \& Floquet, M. 1998, A\&A, 335, 565

Irvine, N. J. 1975, ApJ, 196, 773

Ivanova, D. V., Sakhibullin, N. A., \& Shimanskii, V. V. 2004, Astron. Rep., 48, 476

Jaschek, C., \& Jaschek, M. 1992, A\&AS, 95, 535

Jaschek, M., Jaschek, C., Hubert-Delplace, A.-M., \& Hubert, H. 1980, A\&AS, 42, 103

Jaschek, M., Slettebak, A., \& Jaschek, C. 1981, Be star terminology, Be Star Newsletter, 4, 9

Kaiser, D. 1987, A\&AS, 67, 203

Kogure, T., \& Leung, K.-C. 2007, The Seventh Pacific Rim Conference on Stellar Astrophysics, eds. Y. W. Kang, H.-W. Lee, K.-C. Leung, \& K.-S. Cheng, ASP Conf. Ser., 362, 260

Koubský, P., Kotková, L., Votruba, V., et al. 2012, ArXiv e-prints [arXiv: 1205.2259]

Krtička, J., Owocki, S. P., \& Meynet, G. 2011, A\&A, 527, A84

Kucewicz, B. 1967, Inform. Bull. Southern Hemisphere, 11, 34

Kuchner, M. J., Silverberg, S. M., Bans, A. S., et al. 2016, ApJ, 830, 84

Kurucz, R. 1969, in Theory and Observation of Normal Stellar Atmospheres, ed. O. Gingerich, 375

Kwan, J., \& Fischer, W. 2011, MNRAS, 411, 2383

Lanz, T., \& Hubeny, I. 2007, ApJS, 169, 83

Levenhagen, R. S., \& Leister, N. V. 2006, MNRAS, 371, 252

Mathew, B., Banerjee, D. P. K., Subramaniam, A., \& Ashok, N. M. 2012, ApJ, 753,13

Mennickent, R. E., \& Vogt, N. 1988, A\&AS, 74, 497

Merrill, P. W., \& Burwell, C. G. 1943, ApJ, 98, 153

Moto'oka, K., \& Itoh, Y. 2013, RA\&A, 13, 1189

Neiner, C., Hubert, A.-M., \& Catala, C. 2005, ApJS, 156, 237

Okazaki, A. T. 1997, A\&A, 318, 548

Polidan, R. S. 1976, in Be and Shell Stars, ed. A. Slettebak, IAU Symp., 70, 401

Polidan, R. S., \& Peters, G. J. 1976, in Be and Shell Stars, ed. A. Slettebak, IAU Symp., 70, 59

Porter, J. M., \& Rivinius, T. 2003, PASP, 115, 1153

Rivinius, T., Carciofi, A. C., \& Martayan, C. 2013a, A\&ARv, 21, 69

Rivinius, T., Townsend, R. H. D., Kochukhov, O., et al. 2013b, MNRAS, 429, 177

Slettebak, A., Wagner, R. M., \& Bertram, R. 1997, PASP, 109, 1

Stagg, C. 1983, Information Bulletin on Variable Stars, 2376

Tolbert, C. R. 1964, ApJ, 139, 1105

Vernet, J., Dekker, H., D’Odorico, S., et al. 2011, A\&A, 536, A105

Vieira, R. G., Carciofi, A. C., Bjorkman, J. E., et al. 2017, MNRAS, 464, 3071

Zorec, J., \& Briot, D. 1997, A\&A, 318, 443

Zorec, J., Divan, L., \& Briot, D. 1983, A\&A, 126, 192

Zorec, J., Frémat, Y., \& Cidale, L. 2005, A\&A, 441, 235

Zorec, J., Frémat, Y., Martayan, C., Cidale, L. S., \& Torres, A. F. 2007, in Active OB-Stars: Laboratories for Stellare and Circumstellar Physics, eds. A. T. Okazaki, S. P. Owocki, \& S. Stefl, ASP Conf. Ser., 361, 539

Zorec, J., Cidale, L., Arias, M. L., et al. 2009, A\&A, 501, 297 


\section{Appendix A: Notes on individual Be stars}

In the following, observations of interest are noted for each of the identified $\mathrm{Be}$ stars together with literature values of parameters and stellar rotation, where available, and whether the star is a known or newly identified Be star.

- HIP 11116 (HD 14850). Buscombe (1970) report emission in $\mathrm{H} \beta$. The analysis by Levenhagen \& Leister (2006) yields a spectral type B8 Ve and $T_{\text {eff }}=13500 \pm 550 \mathrm{~K}, \log g=$ $3.60 \pm 0.10$, and $v \sin i=150 \pm 20 \mathrm{kms}^{-1}$. The parameters determined by the BCD method in this work are within the $3 \sigma$ errors determined in that study, but not vice versa.

- HIP 15188 is listed as a B3 Ve star with $v \sin i \approx 130 \mathrm{~km} \mathrm{~s}^{-1}$ in the SIMBAD database. The spectral appearance is in agreement with an early-type star, but the Be star seems inactive at the moment, i.e., there is no trace of circumstellar line emission.

- HIP 23161 (HD 31764, HR 1600). Tolbert (1964) classify the star as a variable in a binary system. They identify the components as B8-7 III and B6-7 IV, suggesting a period of $P=230$ yr. In our spectra, no companion is obvious nor would any detectable radial velocity change be expected for such a long period, but this object is a newly identified weak Be star.

- HIP 25007 (AN Col, HD 35165, HR 1772) is listed as B5 IVnp by Hiltner et al. (1969) and as B5 IVnpe by Mennickent \& Vogt (1988) This star is a strong shell star that shows a clear double Balmer discontinuity; if this discontinuity is not taken into account, it leads to a later spectral type classification. Consequently, we derive the spectral type as B3 IVe-sh, which is in agreement with Levenhagen \& Leister (2006) whose analysis yields $T_{\text {eff }}=21500 \pm 500 \mathrm{~K}$ and $\log g=3.77 \pm 0.10$ dex with a high $v \sin i=350 \pm 23 \mathrm{~km} \mathrm{~s}^{-1}$. The spectral variability of this object seems to be due to a long-term $V / R$ cycle type behavior.

- HIP 25690 (HD 37027) is a newly identified weak late-type Be star.

- HIP 25950 (HD 36408, HR 1847) is a very narrow lined star that shows no trace of emission in the X-shooter data. The data is unsuitable for BCD parameter determination. This object is a well-separated double star and Harrington \& Kuhn (2009) classify one component as B7 IIIe. The star observed by X-shooter was possibly the other, non-Be component, judging from the spectra shown by Harrington \& Kuhn (2009). As this is not certain, however, we kept this star on the list.

- HIP 26368 (HD 37935, HR 1960) is derived as a spectral type B9.5 Ve according to Carrier et al. (2002).

- HIP 26964 (V731 Tau, HD 37967, HR 1961) is derived as a spectral type of $\mathrm{B} 2.5 \mathrm{Ve}$ as reported in Bhatt et al. (1984); Goraya \& Tur (1988) classify the star as B3 V, $T_{\text {eff }}=$ $21000 \mathrm{~K}, \log g=4.0$, while Frémat et al. (2005) determine $T_{\text {eff }}=16543 \pm 264 \mathrm{~K}$ and $\log g=3.850 \pm 0.041$ and $v \sin i=210 \pm 10 \mathrm{~km} \mathrm{~s}^{-1}$. The discrepancy is probably due to its double BD, filled in by emission, as we yield the same values as Frémat et al. (2005), i.e., a stronger BD when taking into account the doubling. The Balmer emission lines have a very narrow, single peaked appearance, supporting a pole-on star designation, even if the $v \sin i$ seems high for that.

- HIP 28561 (HD 40724, HR 2116) is the brightest newly identified Be star among the ABE sample of Chojnowski et al. (2015). This object shows a double BD in emission and strong IR Ca II triplet emission, and the UVB range shows a clear composite spectrum, i.e., this star is a binary. Whether it is a classical Be star or an interacting binary cannot be decided with the data at hand.

- HIP 29635 (HD 44533) is a newly discovered late-type Be star.

- HIP 31362 (HD 46936) is a newly discovered late-type Be star.

- HIP 32474 (HD 49147, HR 2502) is a newly discovered latetype Be star. This object has been used as a calibrator for interferometry and as a comparison star for polarimetry. These roles are normally not suitable for a Be star. In particular, the emission is variable and there is a clear signature of forming a weak disk in the X-shooter spectra.

- HIP 33509 (HD 51506) is a known early- to mid-type Be star. This object is usually classified earlier than it has been classified in our study and has a double BD in emission.

- HIP 34144 (HD 53296) is a newly discovered late-type Be shell star.

- HIP 36009 (HD 58630) is a newly discovered mid-type Be shell star.

- HIP 37007 (HD 61950, HR 1243) is a newly discovered latetype Be star. This star shows IR Ca II triplet emission, and curiously, while the Balmer and other emission lines do not vary in strength, the IR Ca II triplet emission does.

- HIP 39183 (HD 65804) is a newly discovered late-type Be star. There is a trace signature of the IR Ca II triplet in the Paschen lines it is blended with, but it is impossible to tell whether this is in absorption or emission.

- HIP 39483 (HD 66594, HR 1243) is a newly discovered midtype Be star. This object showed transient activity forming a disk that subsequently decayed. At the limit of the X-shooter resolution one may see a pulsational signature of a low inclination Be star in the $\mathrm{Mg}$ II 4481 line.

- HIP 39595 (HD 66956) is a newly discovered late-type Be star showing the IR Ca II triplet in emission.

- HIP 41085 (HD 70948) is a newly discovered mid-type Be shell star with a double BD.

- HIP 41268 (HD 71255) is a newly discovered late-type Be shell star with a double BD.

- HIP 42060 (HD 72973) is a newly discovered late-type Be star.

- HIP 43073 (HD 208213, HR 3488) is a known late-type $\mathrm{Be}$ star with weak emission that has not been investigated in detail.

- HIP 43114 (AI Pyx, HD 75112) is a newly discovered mid-type Be star. The X-shooter spectrum is overexposed, making a reliable determination of the BCD parameters impossible. In the data acquired by Dall et al. (2007), which is available from the ESO archive, the same $\mathrm{H} \alpha$ signature is present. But these authors classify the star as B5 V, rather than a Be star. The Mg II 4481 profile is curious in both $\mathrm{X}$-shooter and HARPS spectra, hence the star might be a binary.

- HIP 44423 (HD 77907, HR 3611) is a newly discovered midtype Be star.

- HIP 46329 (HD 81753, HR 3745) is a B6 Ve star according to Mennickent \& Vogt (1988) with $v \sin i=300 \mathrm{~km} \mathrm{~s}^{-1}$. Kucewicz (1967) report the spectral type as B5 Ve. In the $\mathrm{X}$-shooter spectra the star shows clear disk growth and may show pulsational signature in the Mg II 4481 profiles.

- HIP 47868 (HD 84567, HR 38780) is a known early-type Be star, discovered by Ghosh et al. (1999). The BeSS database gives the spectral type as B0.5IIIne. The BCD parameters of this star are outside our grid, both in $D_{\star}$ and in $\lambda_{1}$, which may indicate a higher luminosity class. The 
luminosity classification for very early B-type stars with rotationally broadened lines is tricky, as the differences between luminosity classes Ib to about III or even IV are very small. The star may therefore not be a classical Be star, but could be a supergiant with a rotationally modified wind, such as, for instance, $\gamma$ Ara.

- HIP 47962 (HD 84929) is a newly discovered late-type Be star.

- HIP 48582 (HD 85834) is flagged as an emission line star in SIMBAD. Since this was communicated by C. Martayan on the basis of the same X-shooter data used here, we consider it as a new discovery for the purpose of assessing the statistical impact on the Be star frequency. The star shows strong $V / R$ variability in $\mathrm{H} \alpha$. The observed change takes place in 23 days, making it very likely that the star is either a binary, in which case the secondary affects the disk, or a hierarchical triple, in which case the variability is due to the linear superposition of the RV curve of the components $\mathrm{Ba}+\mathrm{Bb}$. The star has strong IR Ca II triplet in emission and the spectrum also shows a small double BD.

- HIP 48943 (OY Hya, HD 86612, HR 3946) is a well-known Be star of spectral type B4 Ve (Hiltner et al. 1969). The parameter $v \sin i=229 \mathrm{~km} \mathrm{~s}^{-1}$ is given by Zorec et al. (1983), also Briot (1986) determine $v \sin i=230 \mathrm{~km} \mathrm{~s}^{-1}$. The star has a pronounced double BD character. Some of the $\mathrm{H} \alpha$ spectra are overexposed and cross-checking with $\mathrm{H} \beta$ reveals that the apparent variability is purely due to this overexposure. However, in the Paschen regime the variability of the Paschen lines blended with the IR Ca II triplet is far stronger than that of the non-blended lines. Most likely, the IR Ca II triplet is in emission, and variably so.

- HIP 51444 (LX Vel, HD 91188) is a known emission line star. This star is a photometrically variable star, for which Balona (1990) report as the most likely frequency $f=$ $0.684 \mathrm{~d}^{-1}$. In the X-shooter spectra the circumstellar emission is clearly variable and the strength of the central absorption in $\mathrm{H} \alpha$ and its variability in the Paschen lines suggests a shell nature of the star.

- HIP 51491 (HD 91120, HR 4123) is a well-known Be shell star for which the IR Ca II triplet is in emission. But it is interesting to note that while $\mathrm{H} \alpha$ varies in the three available VIS spectra, the Ca II triplet remains constant.

- HIP 51546 (HD 91373) is a newly discovered late-type Be star. No reliable stellar parameters can be given due to overexposure of the UVB continuum.

- HIP 52977 (HD 94097) is a newly discovered mid- to latetype Be star. This object shows some low level of variability in $\mathrm{H} \alpha$ and possibly $\mathrm{Mg}$ II 4481.

- HIP 56393 (HD 100528) is a newly discovered late-type Be star. The Paschen line profiles blended with the IR Ca II triplet look suspiciously different from the other Paschen lines, but not different enough to reach a conclusion.

- HIP 57861 (HD 103077) is a newly discovered mid- to latetype Be star. This object showed clear emission line variability in the X-shooter spectra, and He I 4471 and Mg II 4481 are variable in their line profiles as well.

- HIP 59970 (HD 106965) is a newly discovered late-type Be star. Physical parameters cannot be given since the BCD values are outside our grid. This could be because the star might have a weak double $\mathrm{BD}$, or have $\mathrm{BDs}$ merging into each other without being clearly separated, which we could not unambiguously identify. Indeed, this object seems to be a shell star and the He I 4471 line is surprisingly strong for its spectral type (i.e., height of BD). The IR Ca II triplet is certainly present in absorption, whether there is also an emission component is uncertain, but possible.

- HIP 64501 (HD 114531 is a newly discovered late-type Be star.

- HIP 64867 (HD 115415) is a newly discovered late-type Be star.

- HIP 66339 (HD 118246, GP Vir) is a known mid-type Be shell star. The spectral type of this star is classified as B3e, with $v \sin i$ of $270 \mathrm{~km} \mathrm{~s}^{-1}$, by Halbedel (1996), while Slettebak et al. (1997) classify it as B5 IVe and determine a $v \sin i$ of above $350 \mathrm{~km} \mathrm{~s}^{-1}$. This object shows a clear double $\mathrm{BD}$, favoring the earlier spectral type, i.e., B3 V.

- HIP 66351 (HD 117872) is a newly discovered late-type Be star.

- HIP 68100 (HD 120845) is a newly discovered mid- to latetype low inclination, near to pole-on Be star, with $v \sin i=$ $100 \mathrm{~km} \mathrm{~s}^{-1}$.

- HIP 69429 (HD 124176) is a newly discovered late-type Be star.

- HIP 71668 (CK Cir HD 128293) is a known Be star. The spectral types published range from $\mathrm{B} 5 \mathrm{Ve}$, with $v \sin i=$ $216 \mathrm{~km} \mathrm{~s}^{-1}$ (Balona 1975), to B2 IVe (Jaschek \& Jaschek 1992). The X-shooter spectrum favors the earlier type when looking at the $\mathrm{He} / \mathrm{Mg}$ balance.

- HIP 71974 (HD 129433, HR 5484, 4Lib) is a newly discovered late-type Be star.

- HIP 78375 (HD 143513) is a newly discovered late-type Be shell star. The situation is very similar to HIP 59970; physical parameters cannot be given since the BCD values are outside our grid. This could be because the star has a double BD that we failed to identify. The IR Ca II triplet is certainly present in absorption, whether there is also an emission component is uncertain, but possible.

- HIP 80577 (HD 147747) is a newly discovered late-type Be star, seen at low inclination, with $v \sin i=100 \mathrm{~km} \mathrm{~s}^{-1}$.

- HIP 80820 (HD 148382) is a newly discovered late-type Be star. The $\mathrm{H} \alpha$ emission is slightly variable.

- HIP 81321 (HD 149595) is a newly discovered, very weak late-type Be star. No UVB spectrum is available, but the $\mathrm{H} \alpha$ profile is clearly indicating a Be star.

- HIP 82874 (HD 152541) is a known but little studied latetype Be star.

- HIP 83278 (HD 153608) is a newly discovered late-type Be star.

- HIP 84184, is a newly discovered late-type strong Be shell star. The strength of the shell changed considerably in the Xshooter spectra over about two years, only showing a double $\mathrm{BD}$ when the stronger shell is present.

- HIP 85138 (HD 156709) is a newly discovered late-type $\mathrm{Be}$ star. The $\mathrm{H} \alpha$ emission is slightly variable.

- HIP 85195 (HD 157546, HR 6473) is a newly discovered late-type $\mathrm{Be}$ star. The $\mathrm{H} \alpha$ emission is slightly variable.

- HIP 85566 (HD 158419) is a newly discovered late-type Be star. However, it was reported by Kuchner et al. (2016) as a disk candidate based on its infrared excess, indicating cold dust. It might, therefore, rather be a Herbig Ae star or a $\beta$ Pictoris type object. The IR Ca II triplet is in absorption, but might have an emission component as well.

- HIP 87032 (HD 161734) is classified as an emission line star by Gray \& Corbally (2002). As the flux calibration of the Balmer continuum is obviously wrong, we do not give BCD parameters. The IR Ca II triplet is in emission.

- HIP 87698 (HD 162888) is a newly discovered weak, but obvious late-type Be star. 
- HIP 88172 (V974 Her, HD 164447, HR 6720) is a known Be star.

- HIP 88374 (HD 164716, HR 6732) is a newly discovered late-type $\mathrm{Be}$ star. The $\mathrm{H} \alpha$ emission is clearly variable.

- HIP 89486 (HD 167230) is a newly discovered late-type $\mathrm{Be}$ star. The $\mathrm{H} \alpha$ emission is clearly variable.

- HIP 89500 (HD 167095) is a newly discovered very weak late-type Be star. The only indication for a Be nature is a slight filled in absorption flank of $\mathrm{H} \alpha$. Since this is however not present in another spectrum, this is a good indication for a variable amount of circumstellar material, i.e., a Be star. Computing the difference spectra reveals the usual double peak emission signature.

- HIP 90096 (HD 169033, HR 6881) is classified by Merrill \& Burwell (1943) a B8 Ve; Jaschek et al. (1980) also list this object as a B8 Ve and derive $v \sin i=220 \mathrm{~km} \mathrm{~s}^{-1}$.

- HIP 90509 (HD 165338) is a newly discovered late-type Be star. The IR Ca II triplet is in absorption, but might have an emission component as well.

- HIP 91460 (HD 172054) is a newly discovered late-type Be star.

- HIP 91975 (4 Aql, HD 173370, HR 7040) is a well-known Be star. Irvine (1975) observe weak emission in $\mathrm{H} \alpha$ with a strong central reversal. The IR Ca II triplet is in absorption, but might have an emission component as well.

- HIP 92038 (HD 173375) is a newly discovered late-type $\mathrm{Be}$ star. The emission strongly increased during the observations with $\mathrm{X}$-shooter over about three years to the point at which a double BD became apparent. The IR Ca II triplet is in emission as well, but its strength decreased, i.e., behaved opposite to the Balmer emission.

- HIP 93993 (HD 178075, HR 7246) is a newly discovered late-type Be star. This object shows an extreme pole-on appearance $v \sin i=10 \mathrm{~km} \mathrm{~s}^{-1}$ with only $\mathrm{H} \alpha$ in emission. The IR Ca II triplet is very weakly present in absorption, which is probably photospheric and typical for the late spectral type.

- HIP 94770 (HD 179419) is a known (but largely ignored) late-type Be star, given as B8 Ve by Andersen \& Nordstrom (1983). The change in the $\mathrm{H} \alpha$ is due to slight over exposure in one of the two spectra and not real. The IR Ca II triplet is in emission.
- HIP 94859 (HD 180699) is a newly discovered late-type Be star. The IR Ca II triplet is in absorption and emission might possibly be present as well.

- HIP 94986 (HD 180885, HR 7316) is a newly discovered low inclination early-type Be star. This object is variable in $\mathrm{H} \alpha$ and the emission is not always present. Pulsational variability is clearly seen in $\mathrm{Mg}$ II 4481.

- HIP 95109 (HD 181751) is a newly discovered late-type Be star. The spectral type is classified as B8 by Stagg (1983).

- HIP 96453 (HD 184597) is a newly discovered mid-type Be star. This object shows clear signs of an outburst in one of the two spectra acquired in the VIS arm of X-shooter.

- HIP 99457 (BE Cap, HD 191639, HR 7709) is a known early-type Be star in SIMBAD, even though it is not entirely clear where this was first reported. This star shows a clear and strong outburst event in the X-shooter data.

- HIP 100664 (HD 194244, HR 7803) is a known late-type Be star, first reported by Neiner et al. (2005).

- HIP 104508 (HD 201317) is a newly discovered late-type Be star. The $\mathrm{H} \alpha$ emission shows clear variability.

- HIP 108022 (16 Peg, HD 208057, HR 8356) is a known mid-type Be star. This star was classified as a Be star by Merrill \& Burwell (1943) and listed as MWC 644. No other study since reported Balmer emission in this star and there is no evidence for a Be nature in the $\mathrm{X}$-shooter spectra either.

- HIP 108402 (HD 208612) is a known late-type Be shell star. This object shows both a double BD and the IR Ca II triplet is in emission.

- HIP 108597 (VV PsA HD 208886) is a known late-type Be shell star discovered by Henize (1976), who found $\mathrm{H} \alpha$ to be a very sharp, moderate to weak emission line and $\mathrm{H} \beta$ to be in absorption, from 1949-1952 objective prism plates. It shows both a double BD and the IR Ca II triplet is in emission.

- HIP 108975 (UU PsA, HD 209522, HR 8408) is reported as an early-type Be star by Merrill \& Burwell (1943) as MWC 650. No recent study has found Balmer emission in this star, and there is no evidence for emission in the $\mathrm{X}$-shooter spectra either. 
Appendix B: Projected rotational velocity fits
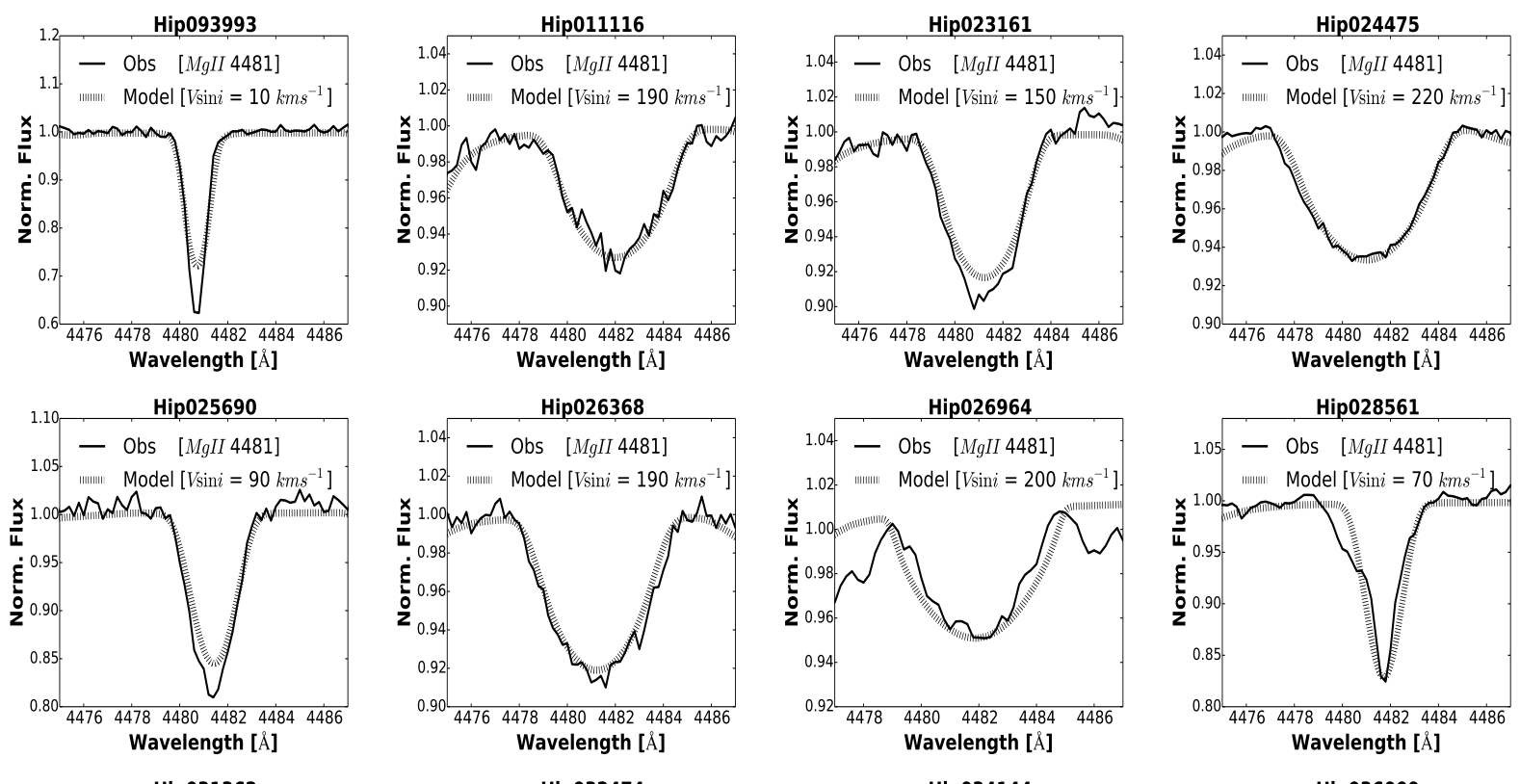

Wavelength $[\AA]$
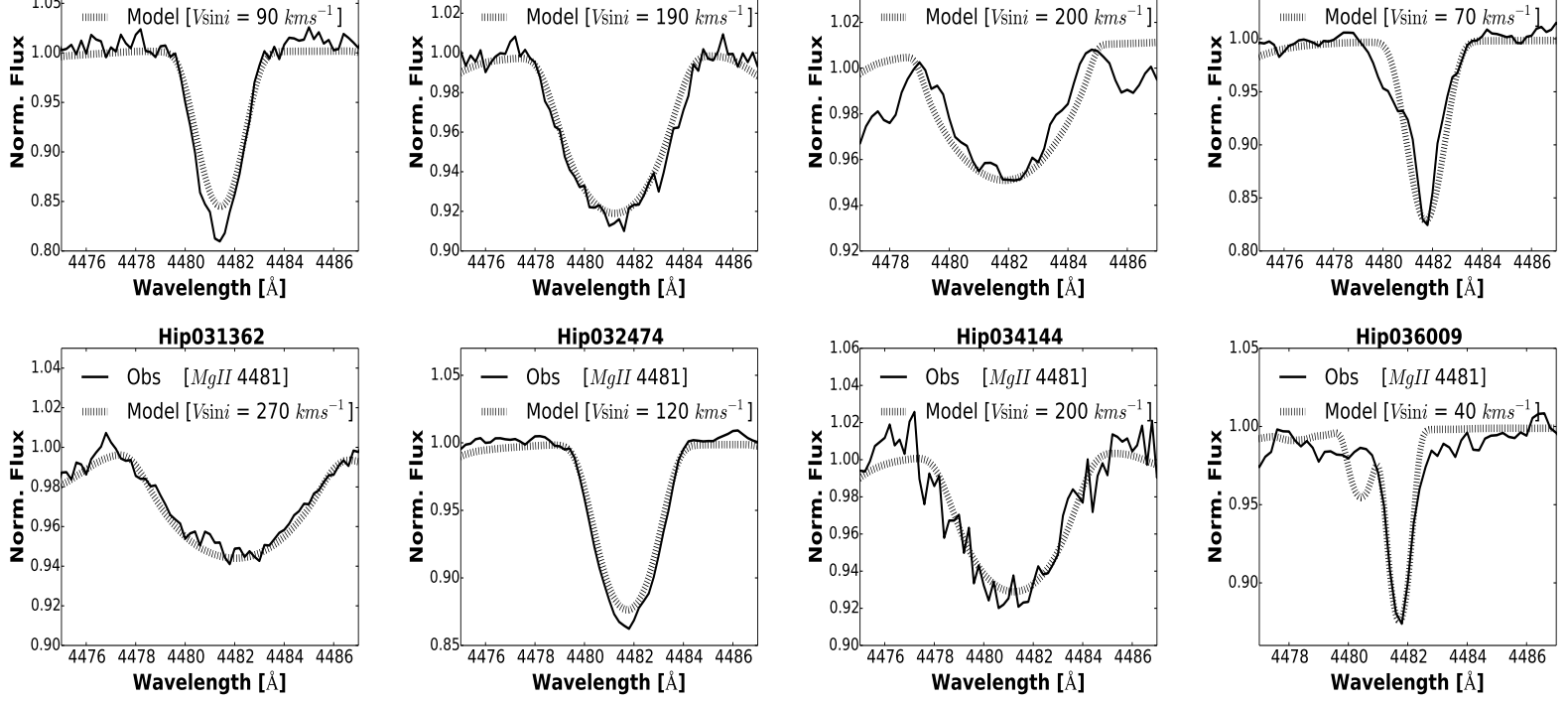

Wavelength $[\AA]$

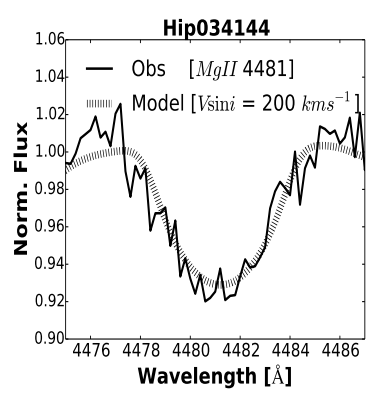

Wavelength $[\AA]$
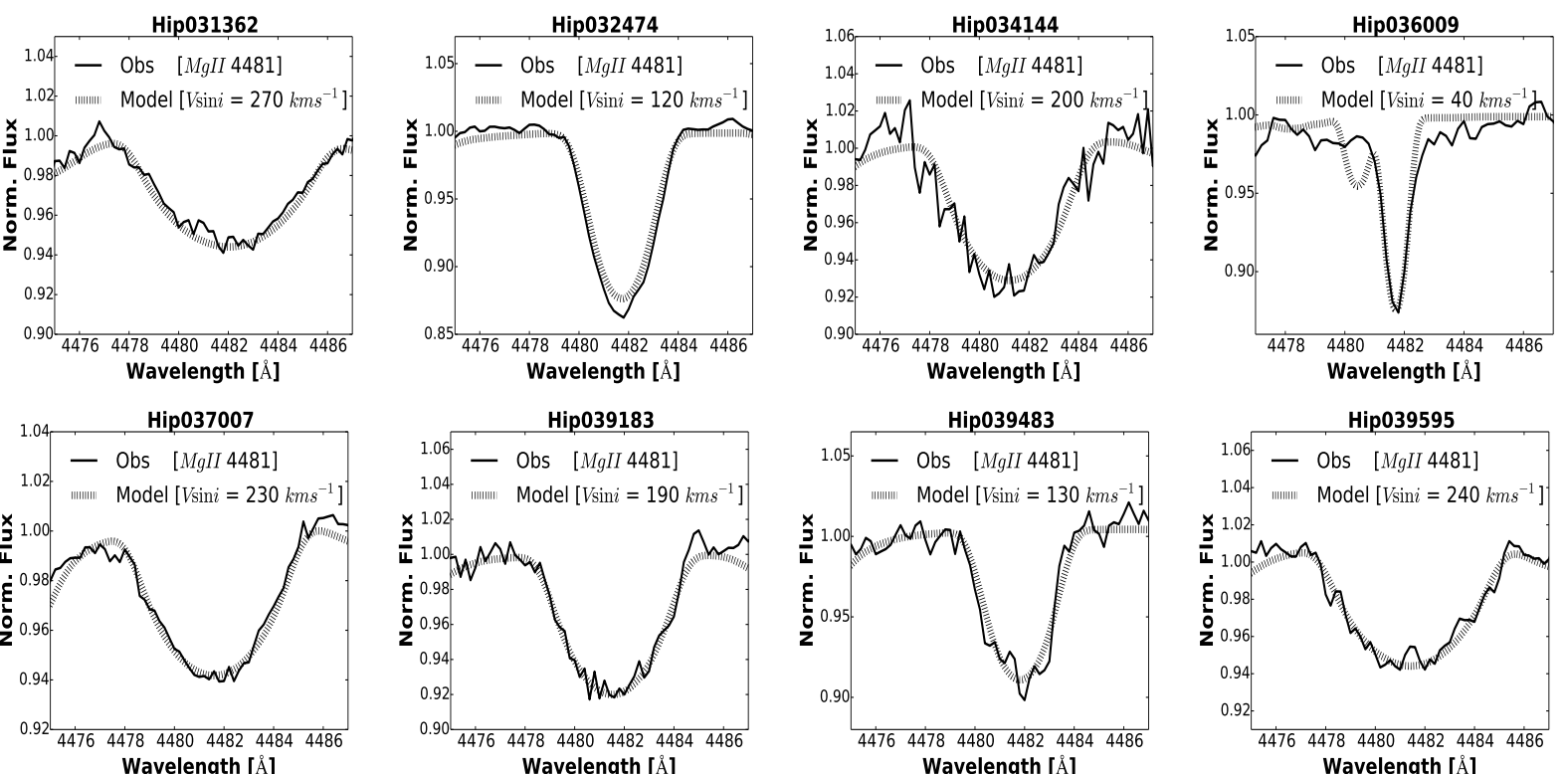

Fig. B.1. Best fit models for projected rotational velocities for the program Be stars, for Mg II 4481 A. Solid lines indicate observed and dotted lines theoretical profiles. 
A. Shokry et al.: Stellar parameters of Be stars observed with X-shooter
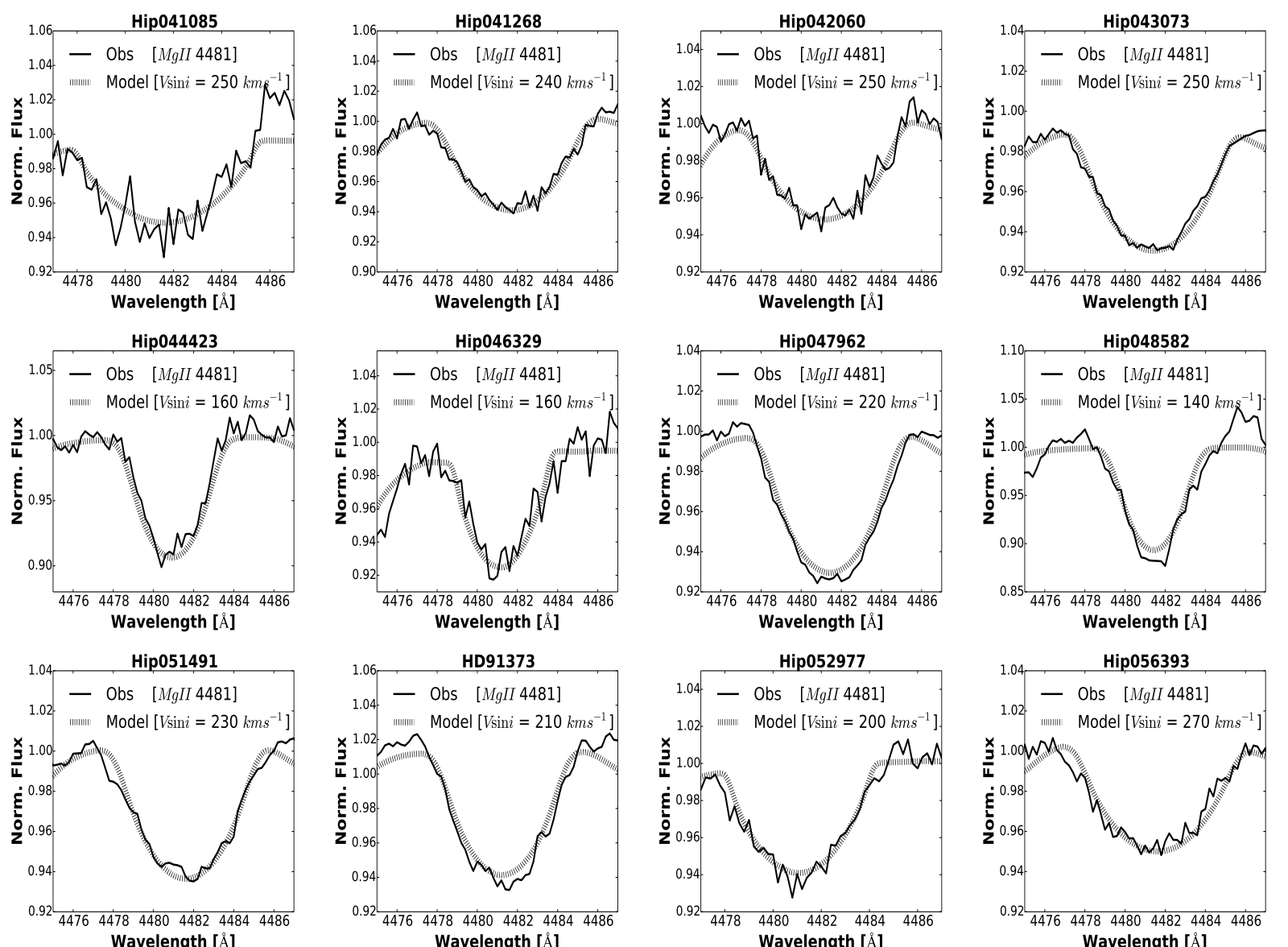

Hip057861

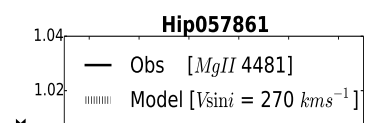

HD106965
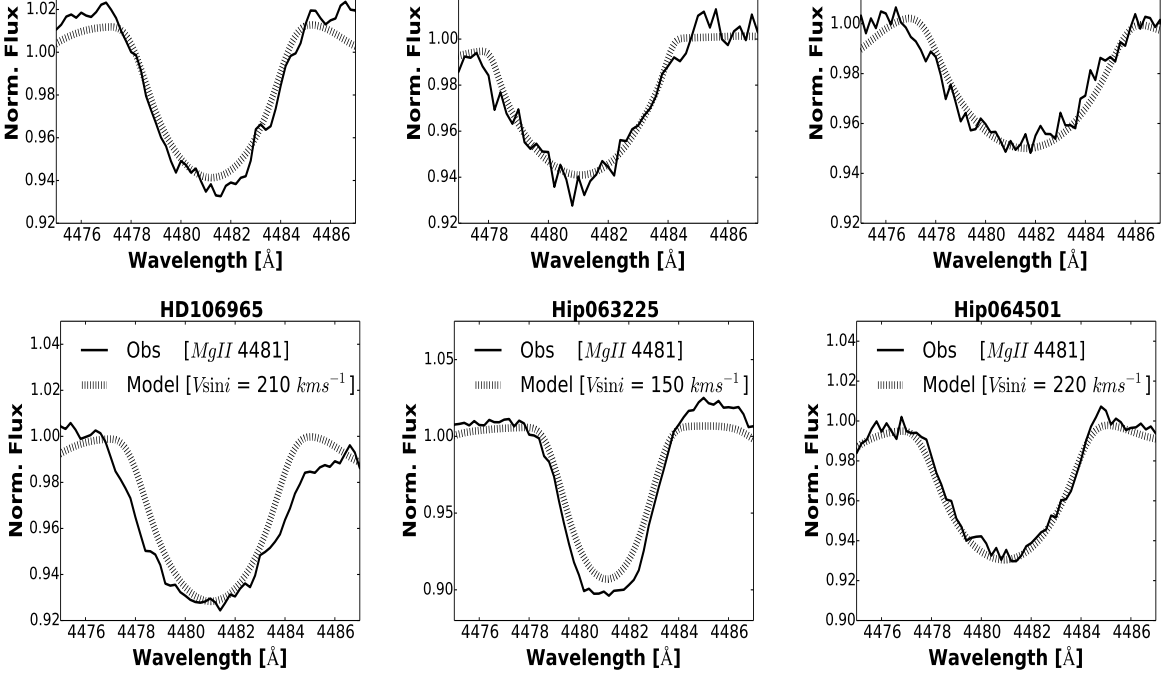

$\stackrel{\mathrm{x}}{\mathrm{z}}_{\mathbf{u}} 1.00$
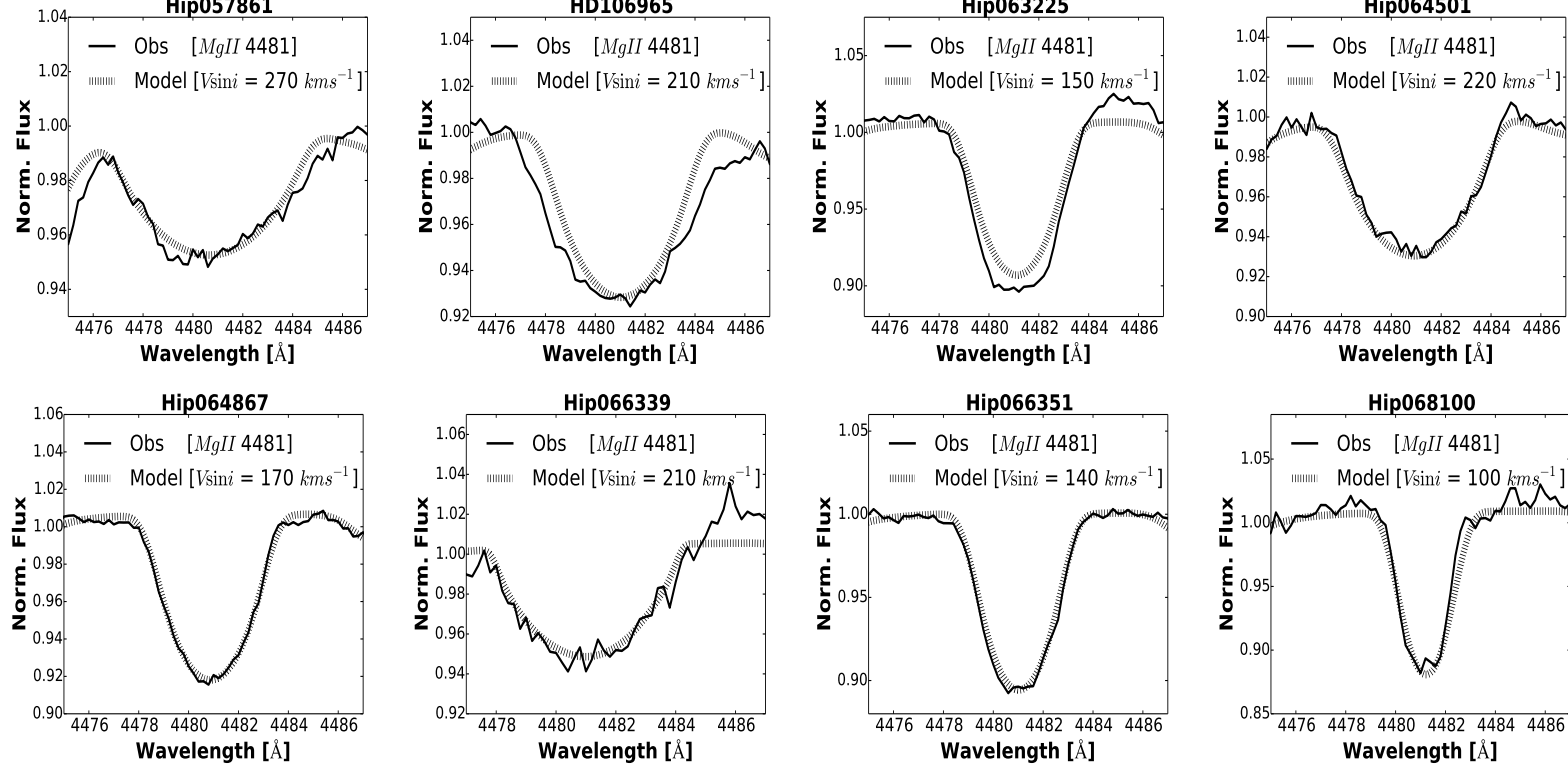

Hip066351
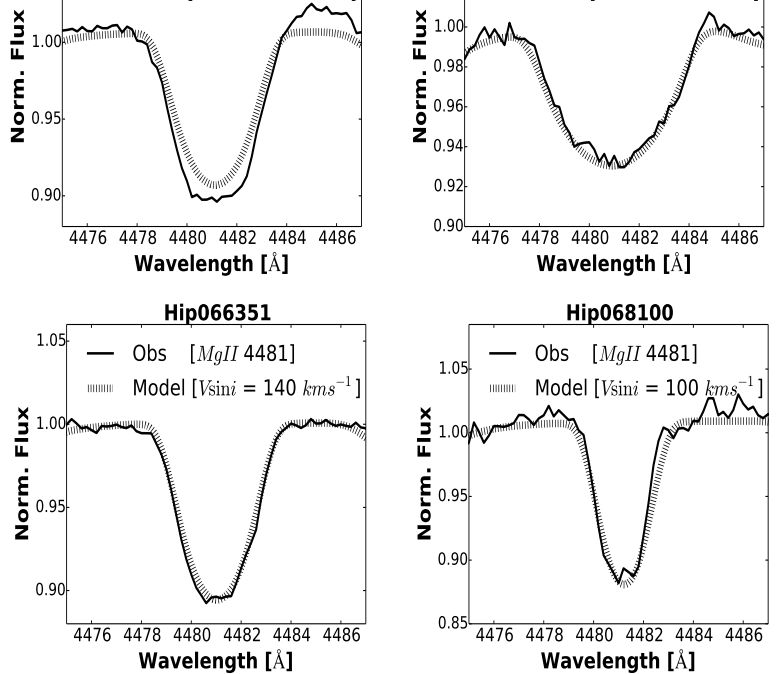

Wavelength $[\AA]$

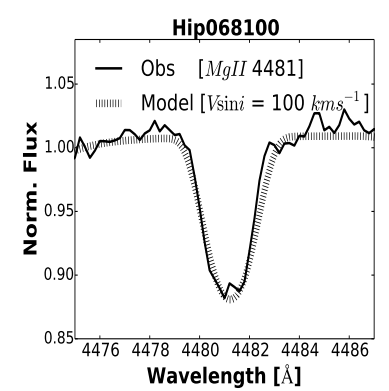

Fig. B.1. continued. 

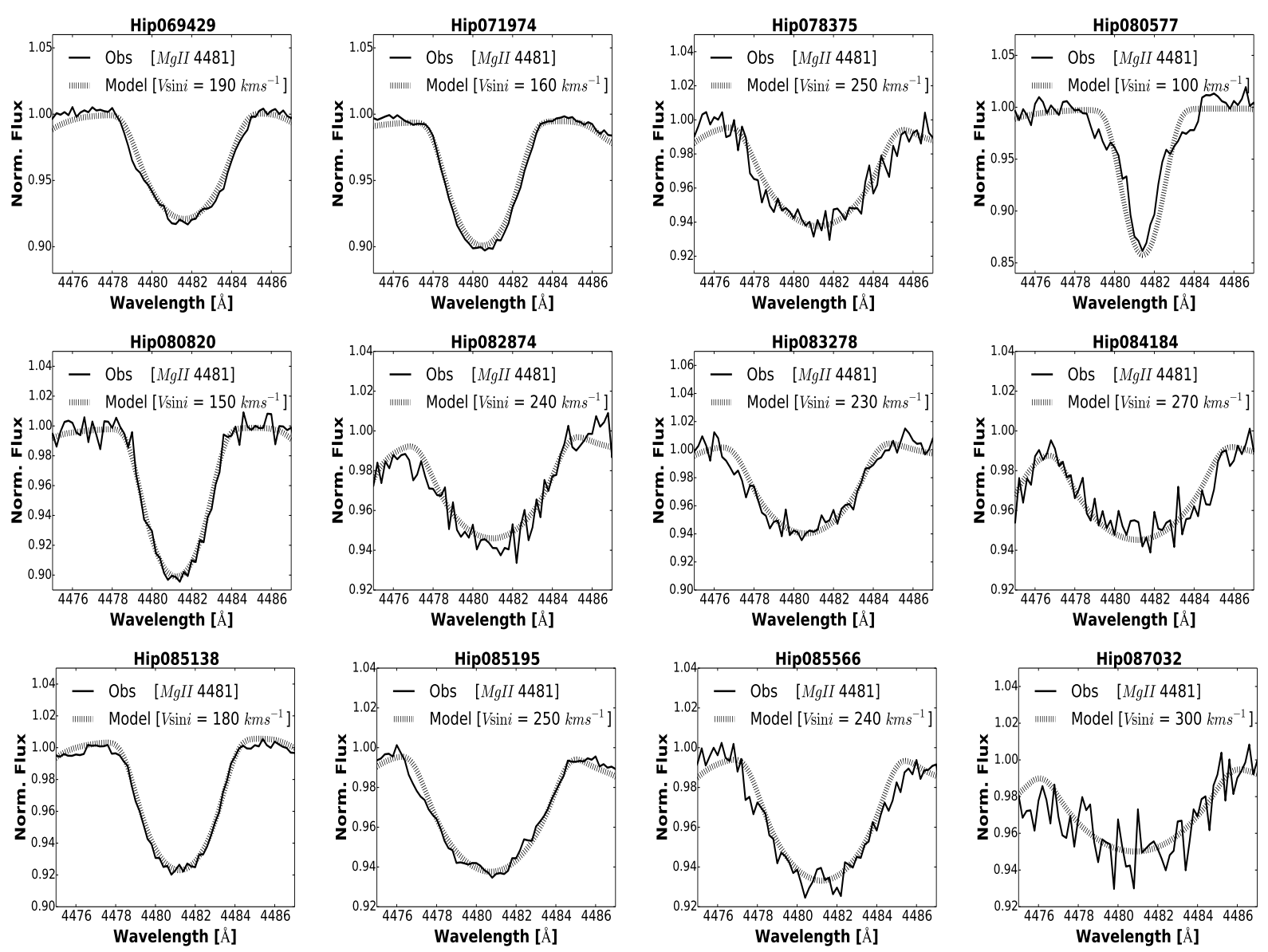

Hip087698
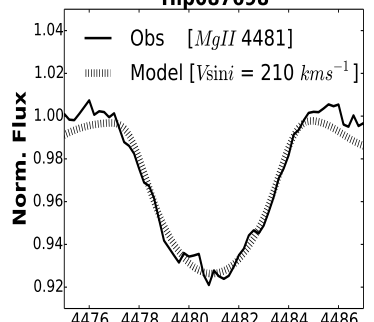

Wavelength $[\AA]$
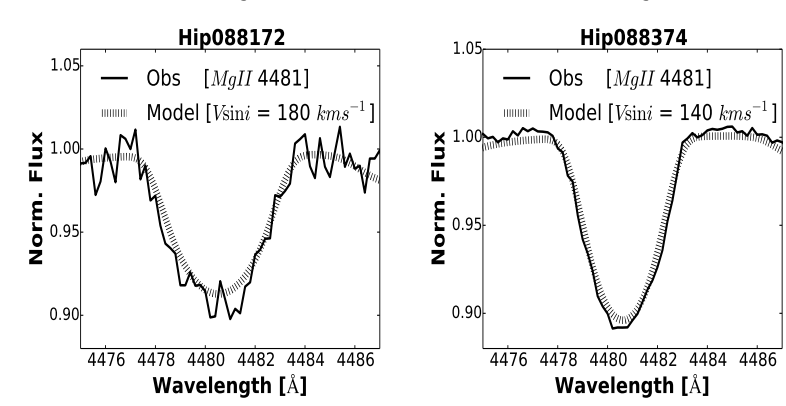

$\begin{array}{lllll}4476 & 4478 \quad 4480 & 4482 & 4484 & 4486\end{array}$

Wavelength [Å]
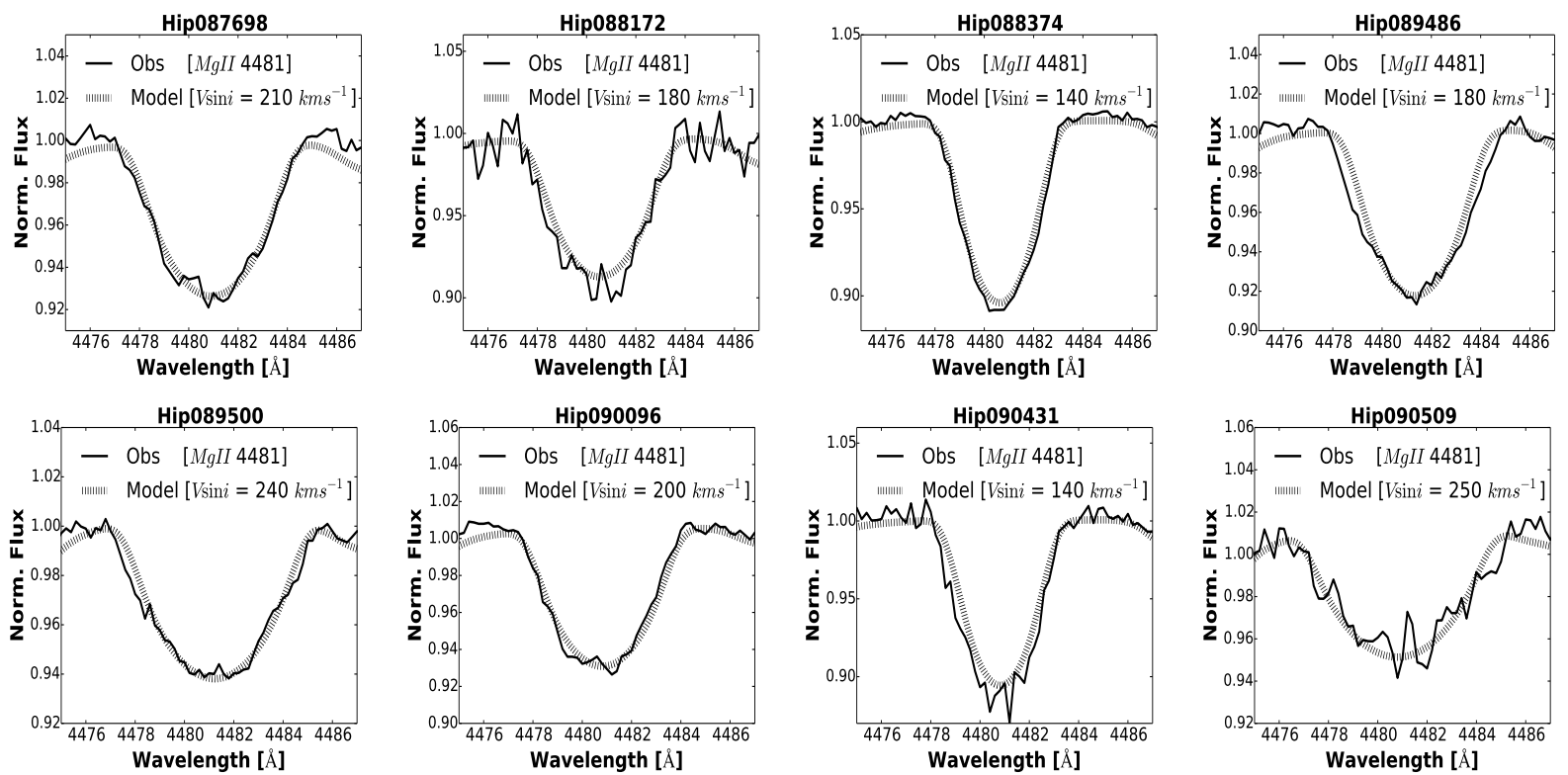

Fig. B.1. continued. 
A. Shokry et al.: Stellar parameters of Be stars observed with X-shooter
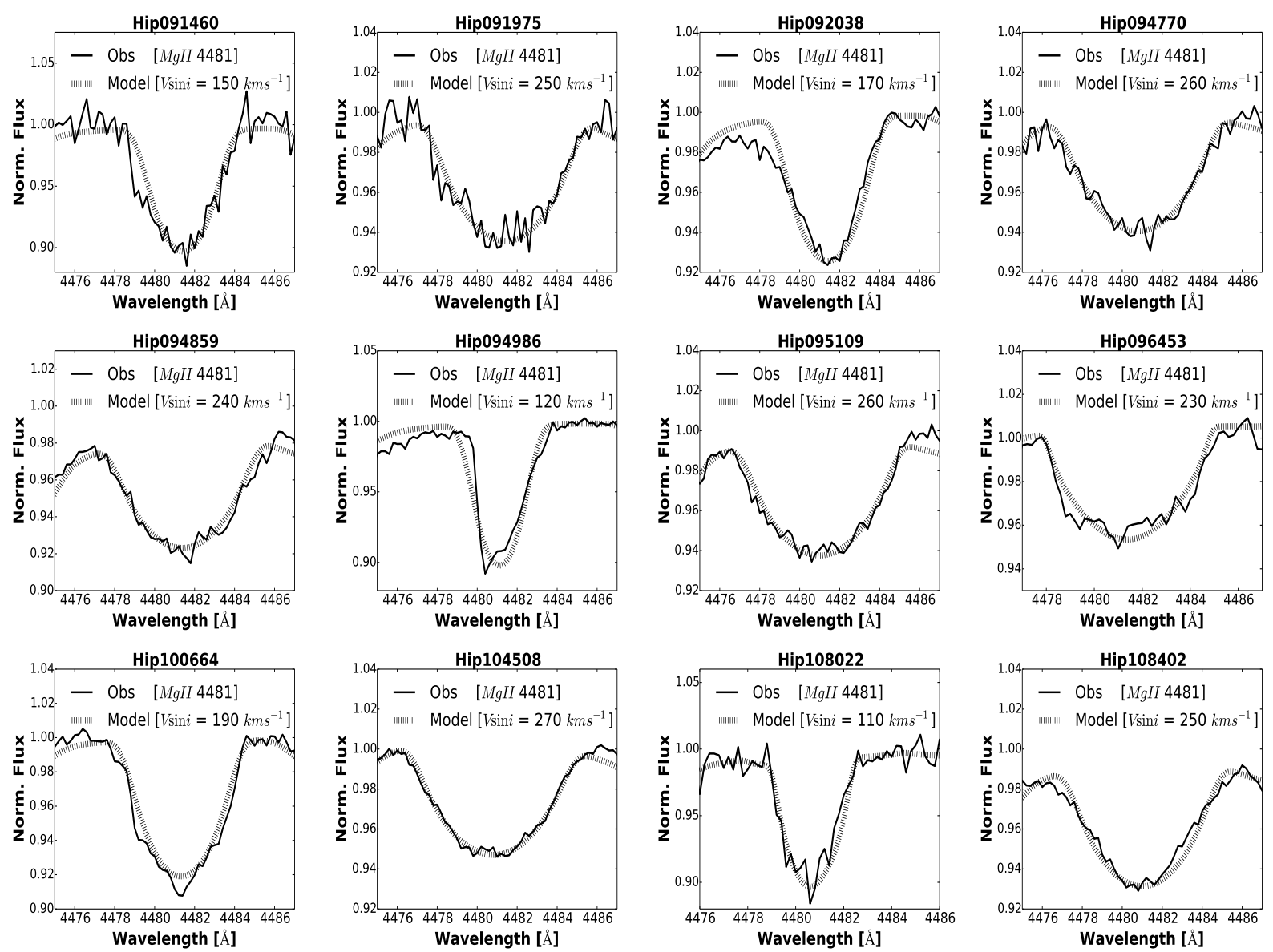

Fig. B.1. continued. 


\section{Appendix C: Spectral appearance of the identified Be stars}

In this Appendix excerpts of the spectra of the identified Be stars are shown, except for HIP 11116, which is shown in Fig. 1. In some cases noisy or otherwise unsuitable spectra were excluded from the plots.

In the top row, from left to right, profiles of $\mathrm{H} \beta, \mathrm{H} \alpha$, the Fe II 5169, and the He I 4471 and $\mathrm{Mg}$ II 4481 lines are shown. These illustrated the presence and variablity of Balmer emission as well as the Balmer decrement, the presence of circumstellar emission or shell absorption in Fe II, and the balance of He vs. Mg may serve as a sanity check on the obtained effective temperatures and spectral types.

In the bottom row, the Balmer discontinuity and the higher lines of the Paschen series, which include the O I 8446 and Ca II triplet lines, are shown.
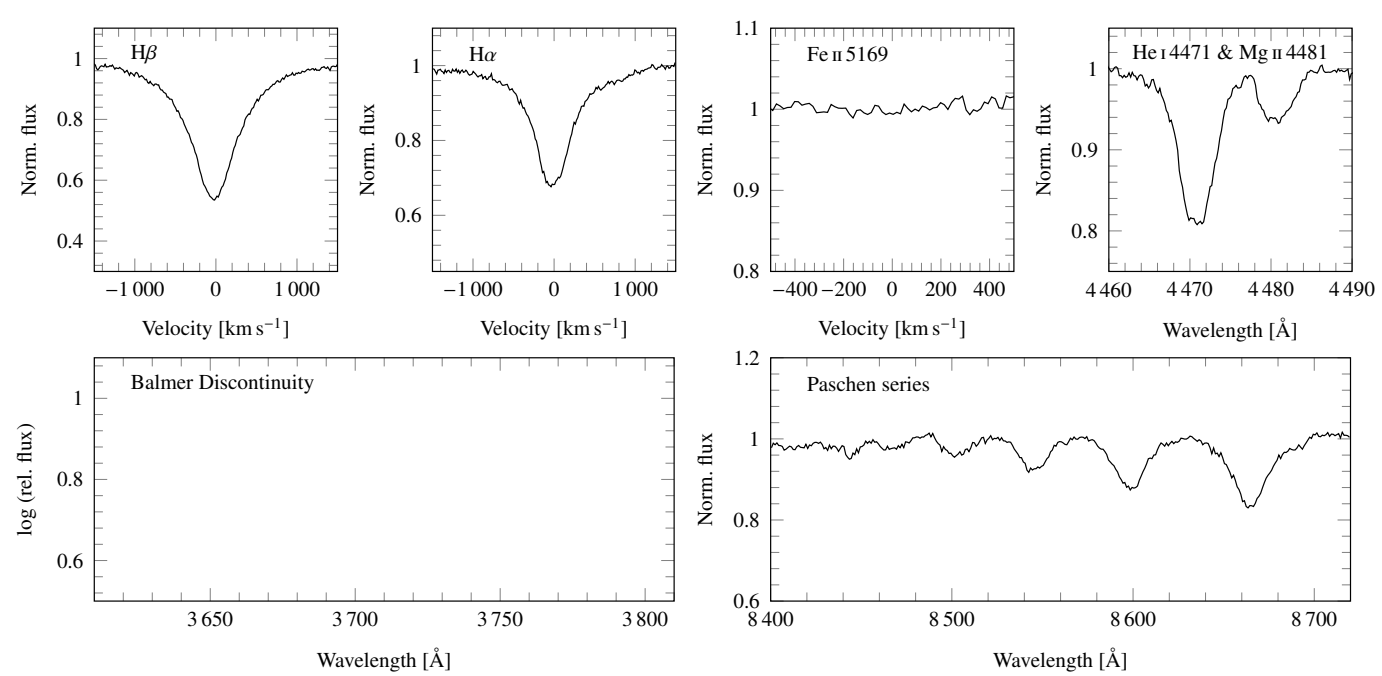

Fig. C.1. Spectrum overview plot for HIP 15188. No usable UVB spectrum is available for this star.
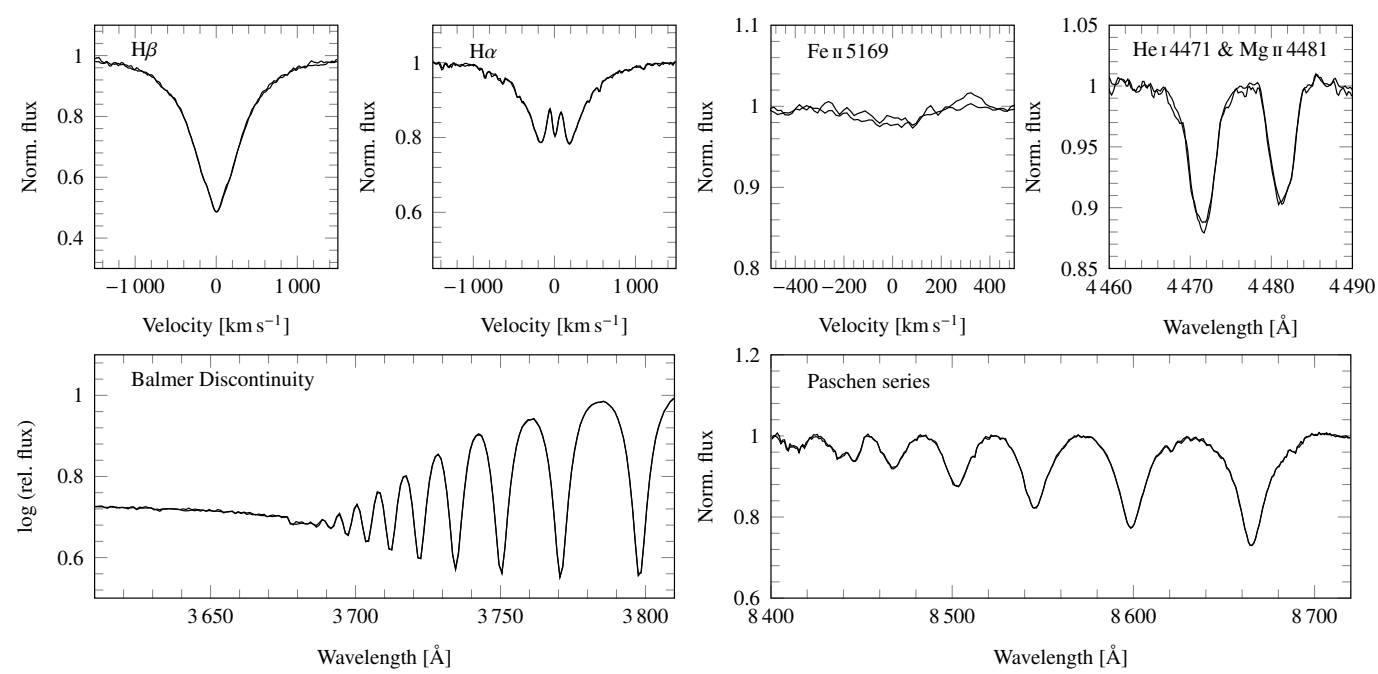

Fig. C.2. Spectrum overview plot for HIP 23161. 
A. Shokry et al.: Stellar parameters of Be stars observed with X-shooter
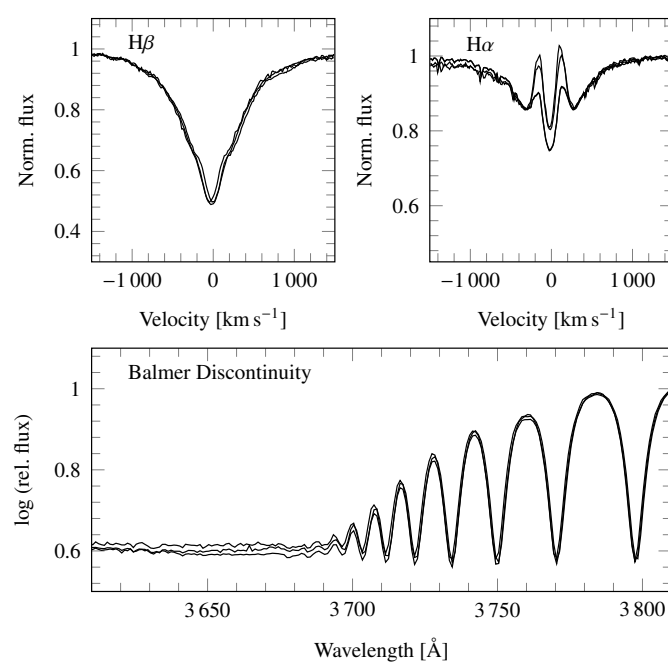

Fig. C.3. Spectrum overview plot for HIP 24475.
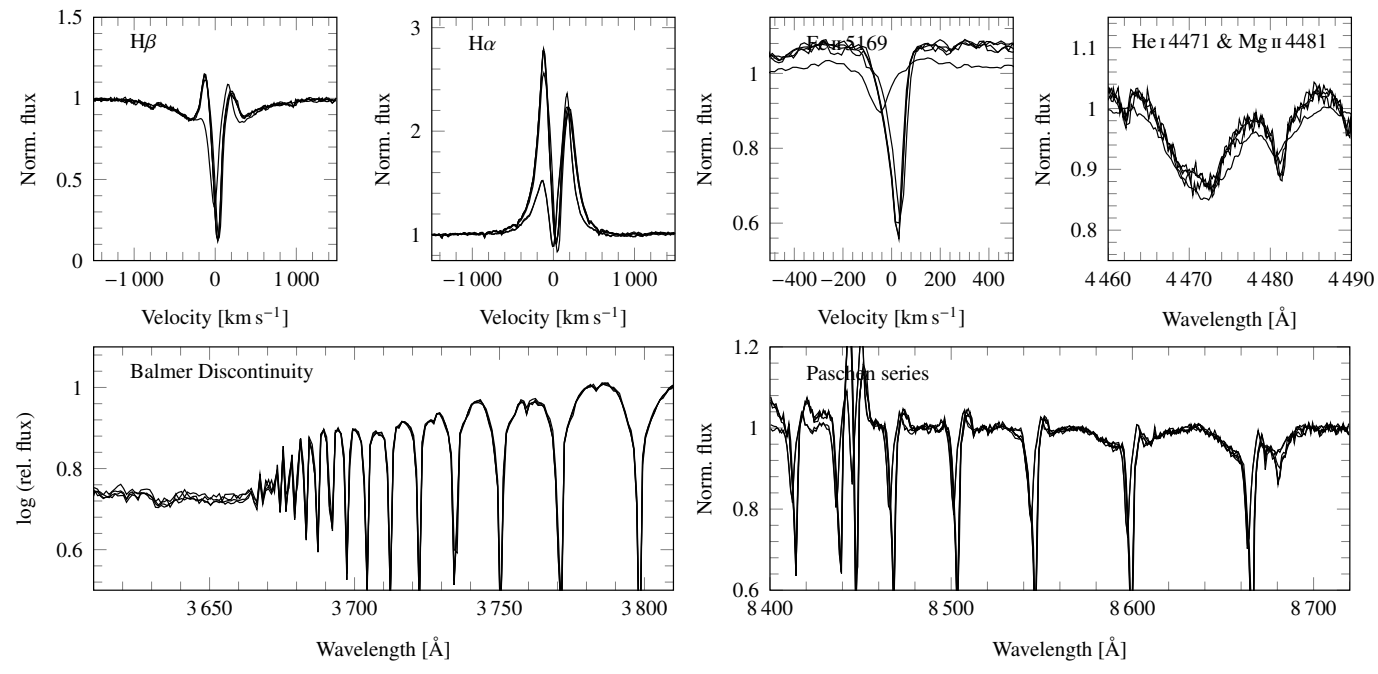

Fig. C.4. Spectrum overview plot for HIP 25007.
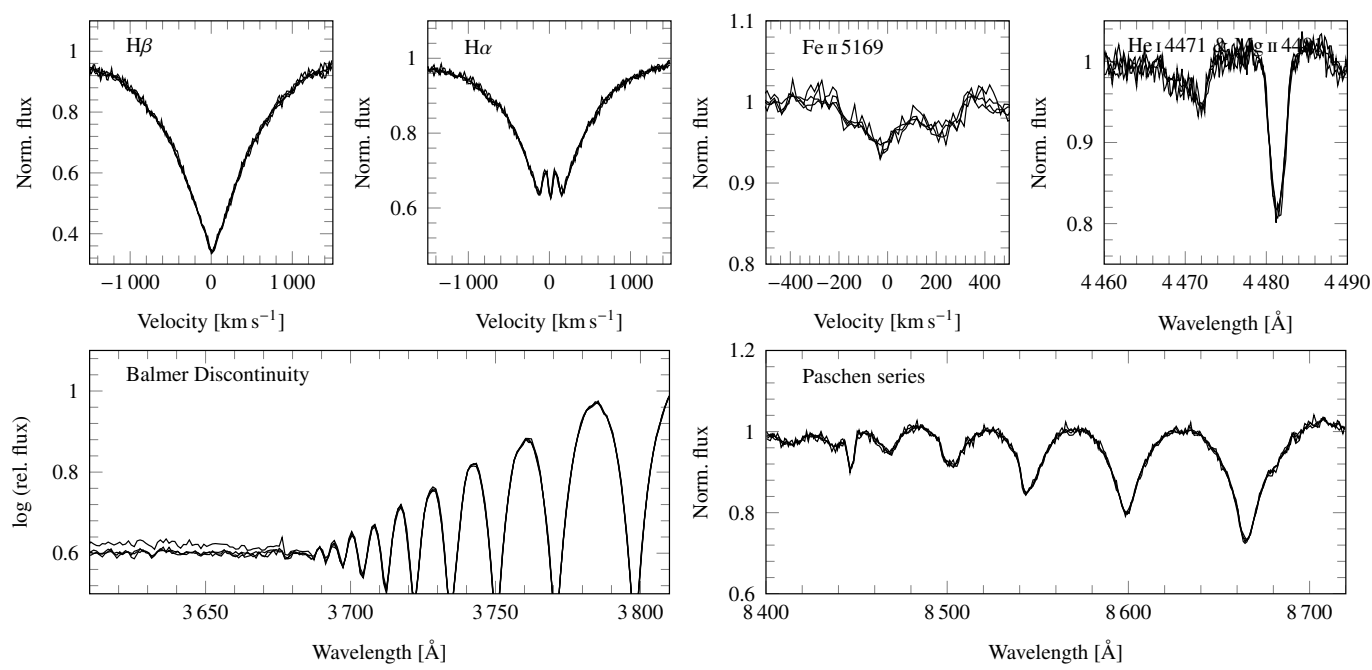

Fig. C.5. Spectrum overview plot for HIP 25690.

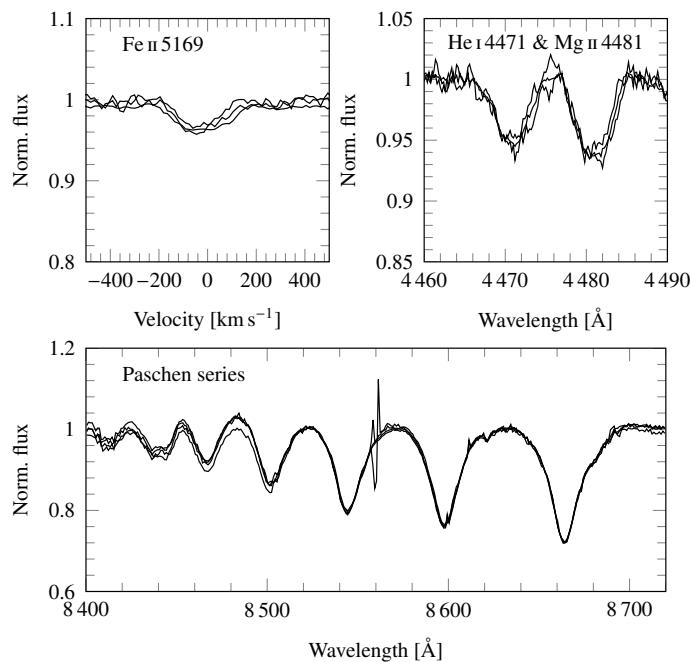


A\&A 609, A108 (2018)
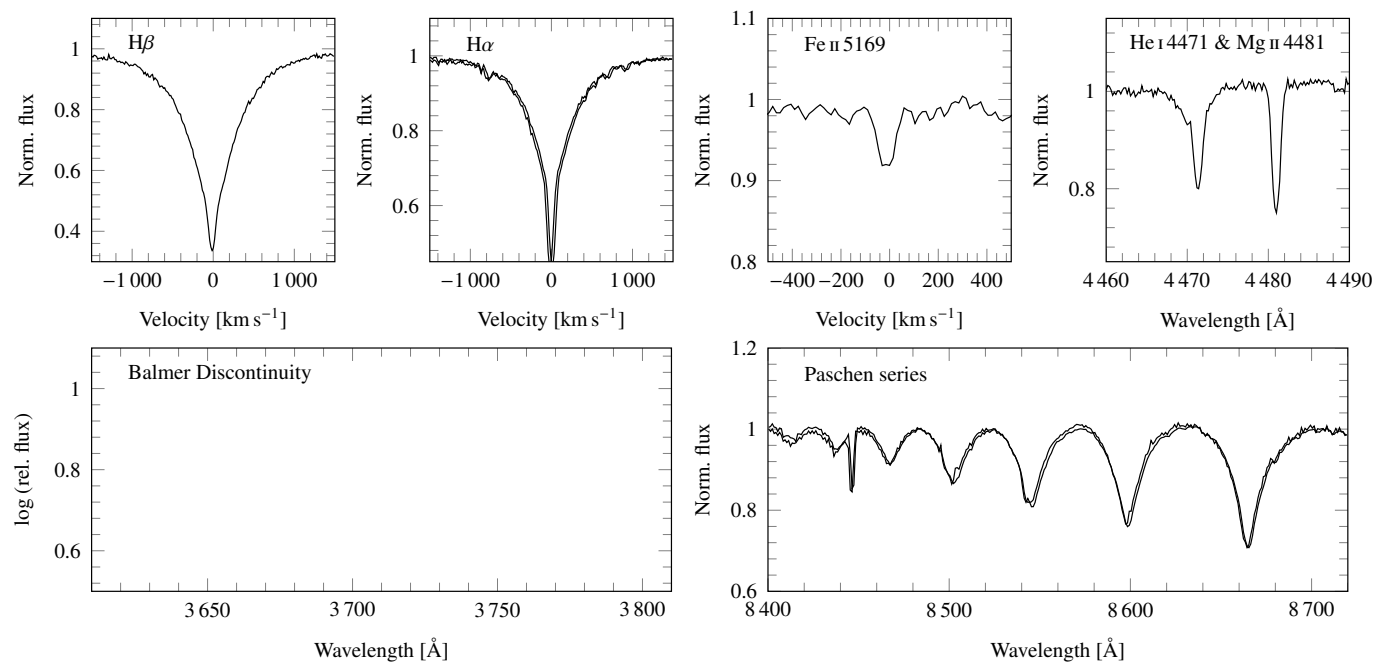

Fig. C.6. Spectrum overview plot for HIP 25950 .
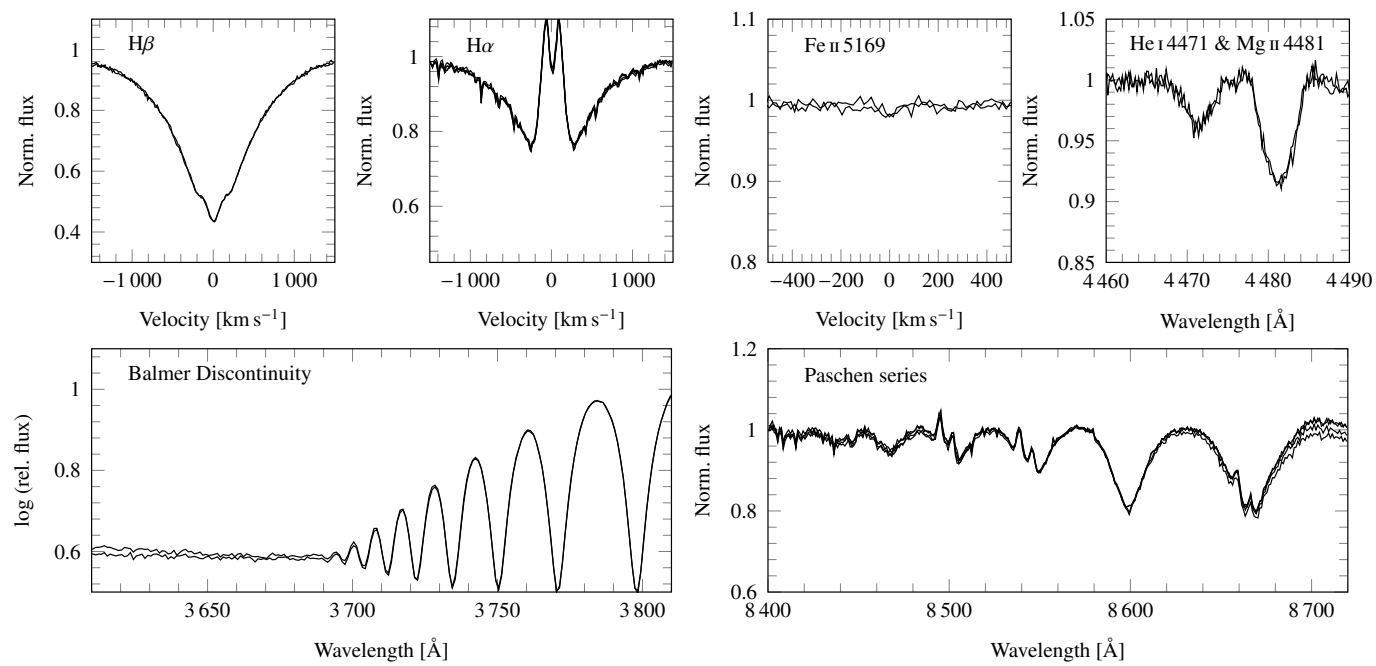

Fig. C.7. Spectrum overview plot for HIP 26368.
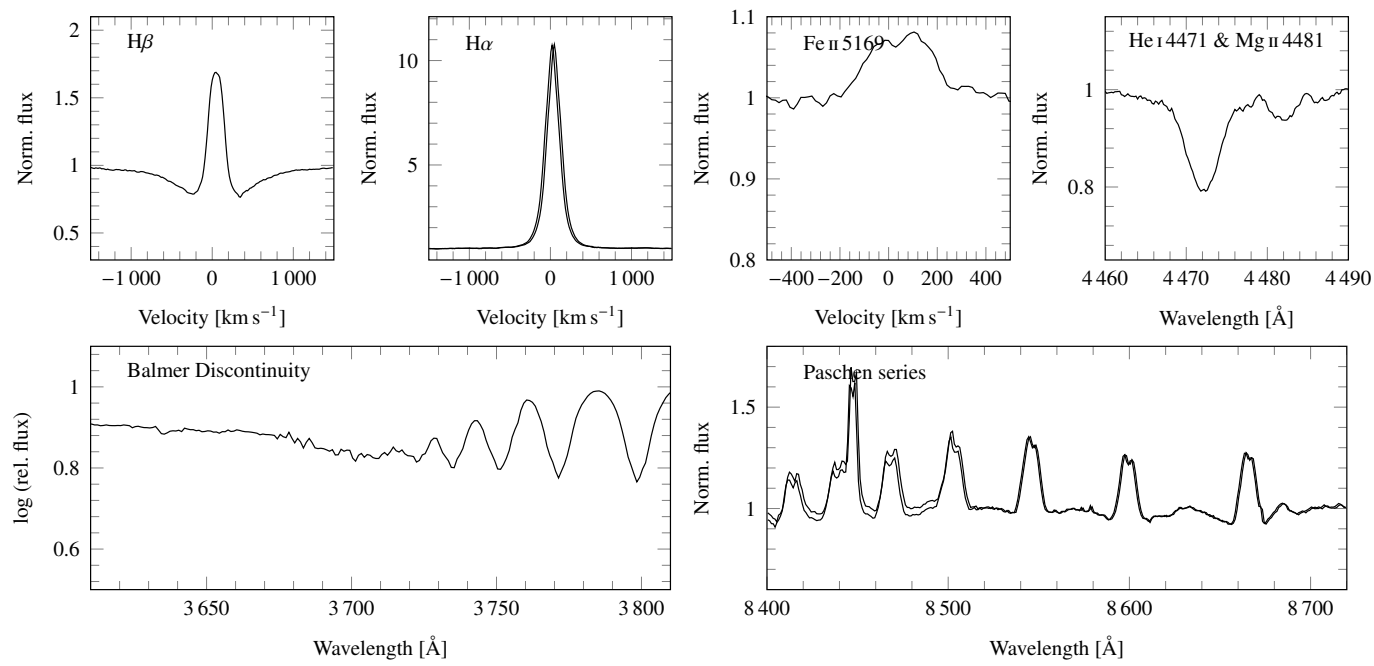

Fig. C.8. Spectrum overview plot for HIP 26964. 
A. Shokry et al.: Stellar parameters of Be stars observed with X-shooter
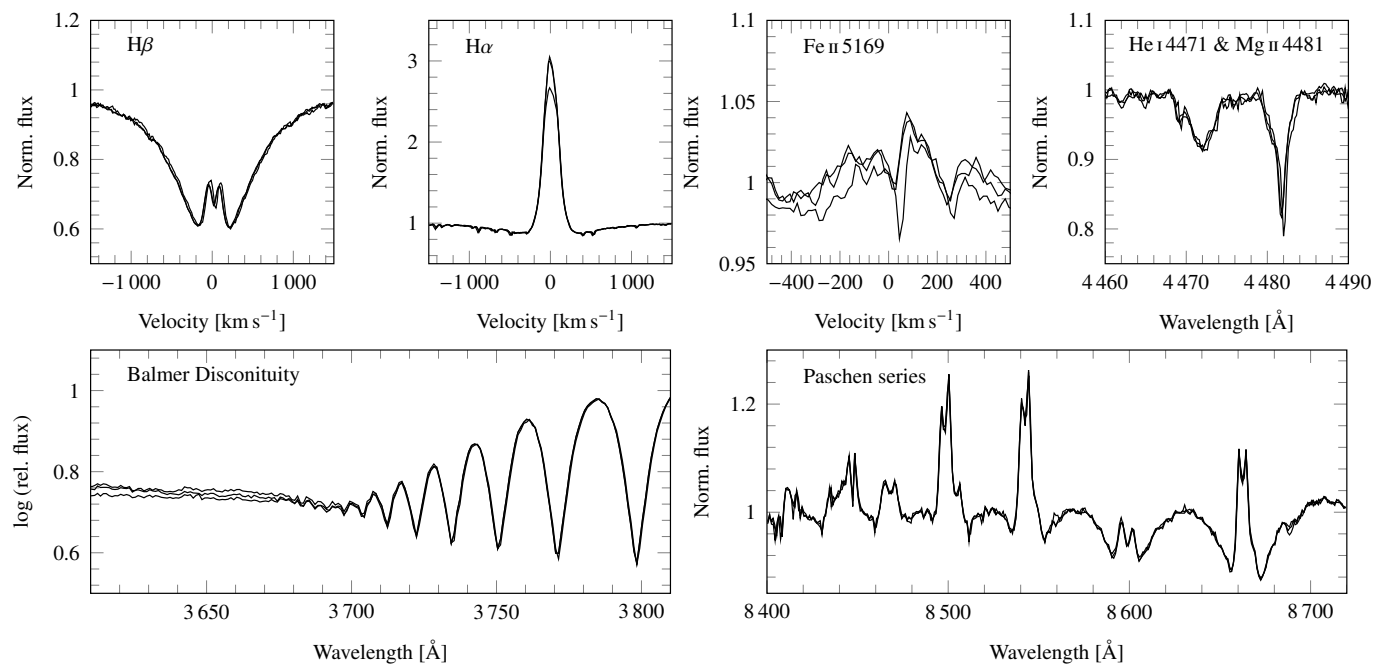

Fig. C.9. Spectrum overview plot for HIP 28561.
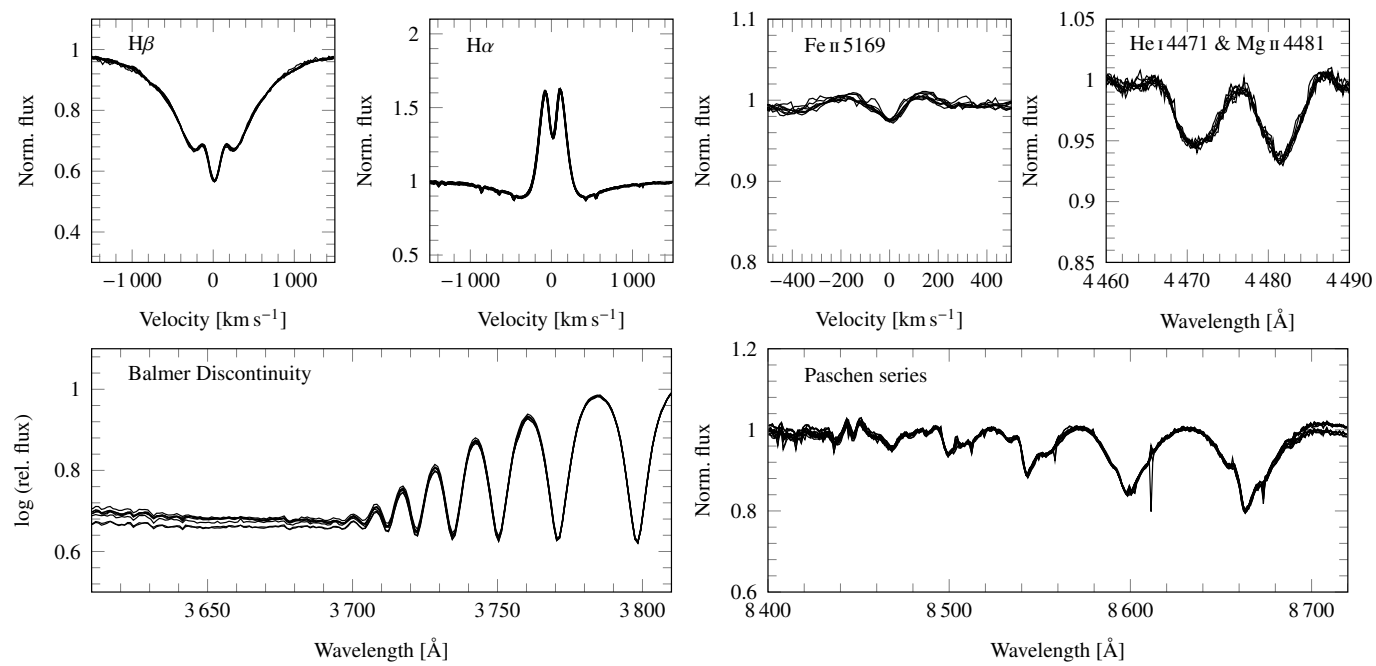

Fig. C.10. Spectrum overview plot for HIP 29635.
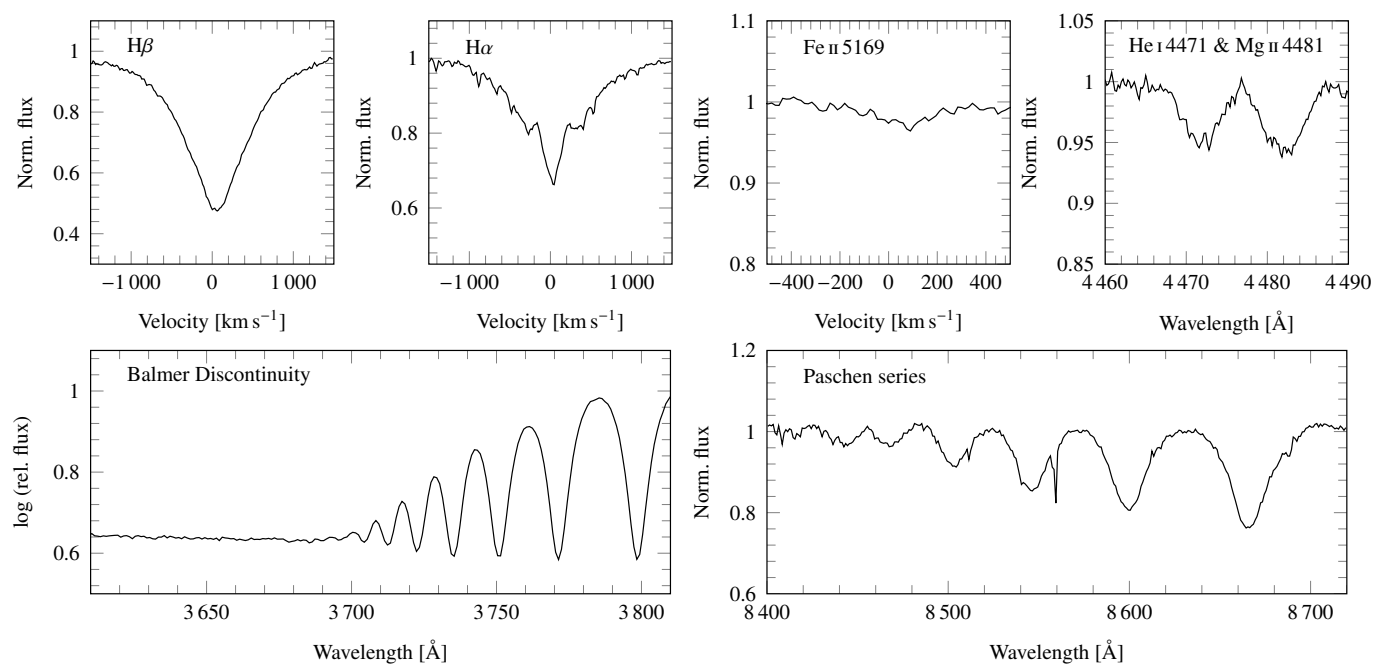

Fig. C.11. Spectrum overview plot for HIP 31362. 

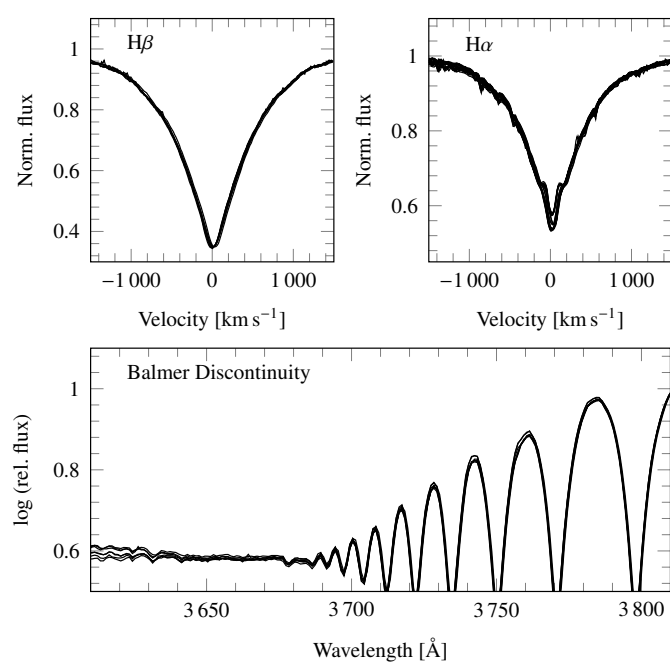
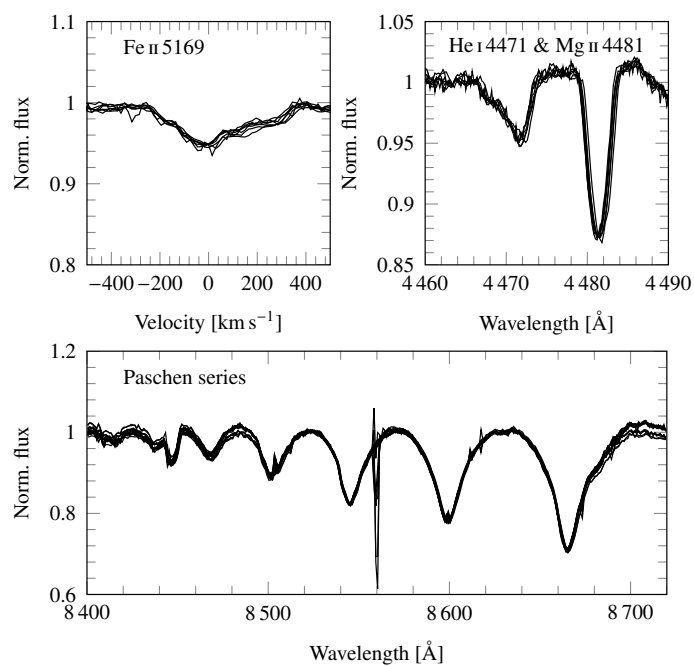

Fig. C.12. Spectrum overview plot for HIP 32474.
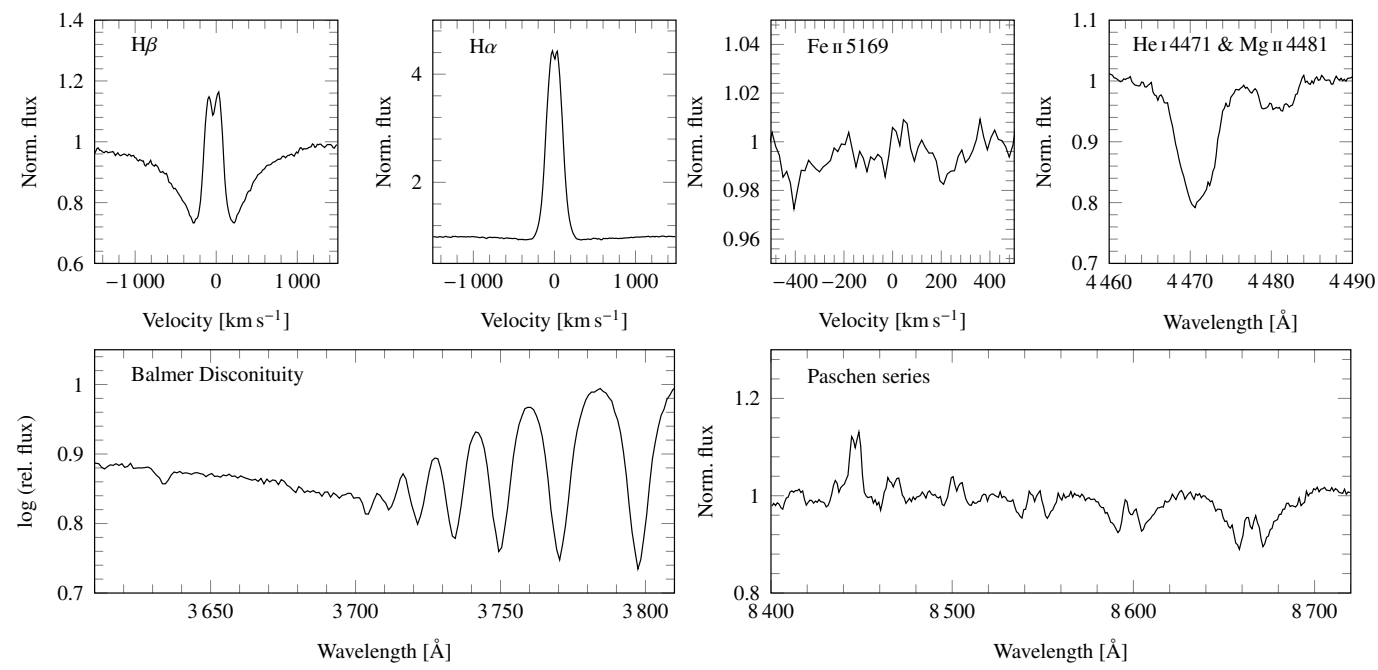

Fig. C.13. Spectrum overview plot for HIP 33509.
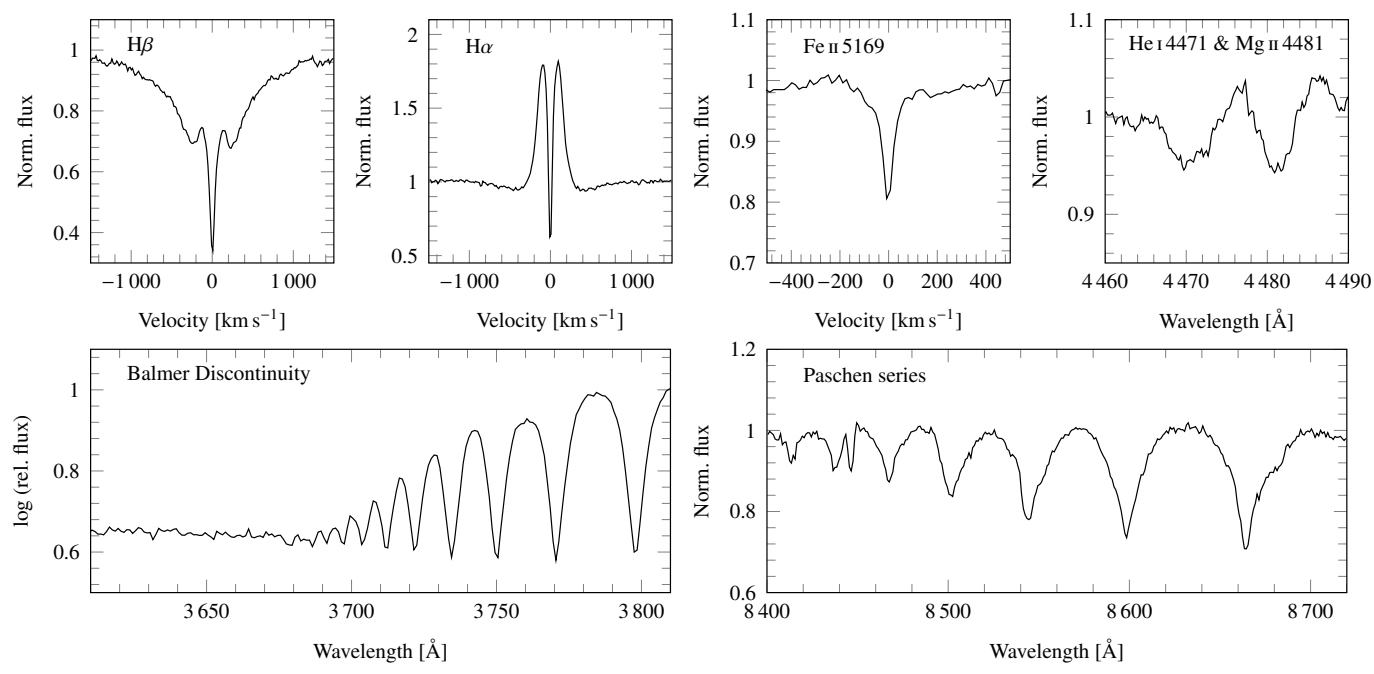

Fig. C.14. Spectrum overview plot for HIP 34144. 
A. Shokry et al.: Stellar parameters of Be stars observed with X-shooter
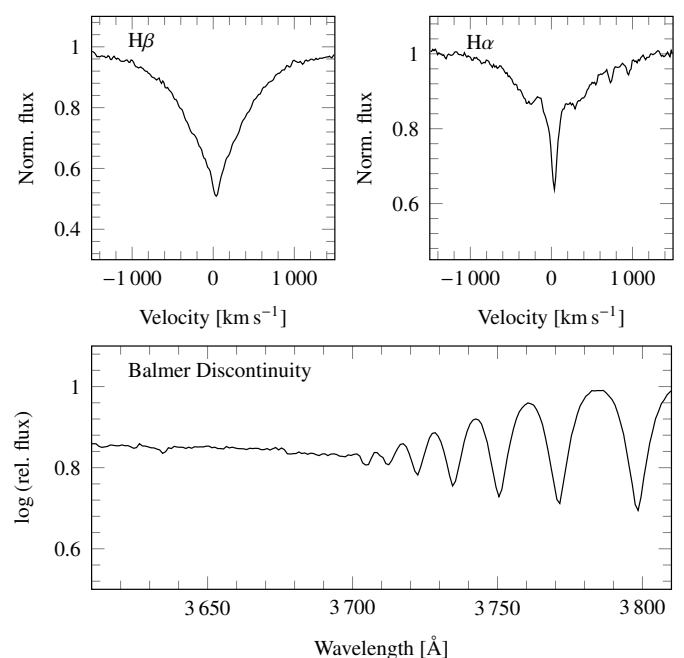

Fig. C.15. Spectrum overview plot for HIP 36009.
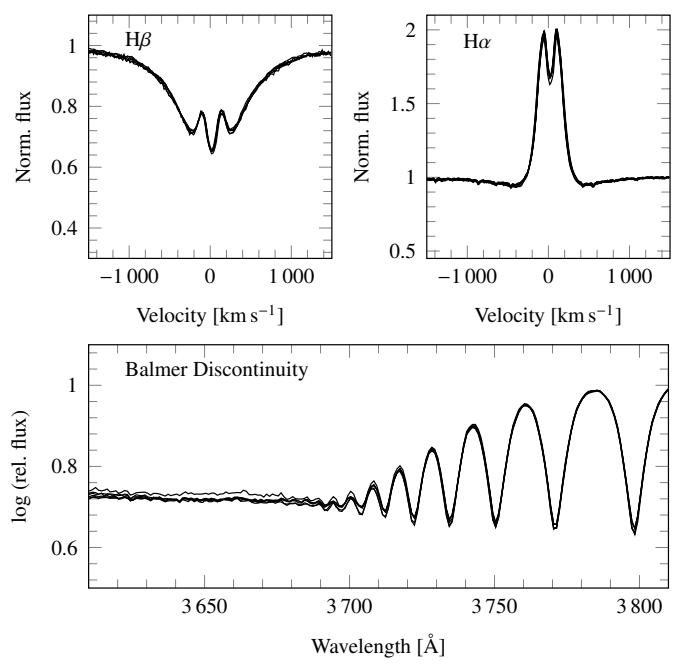

Fig. C.16. Spectrum overview plot for HIP 37007.
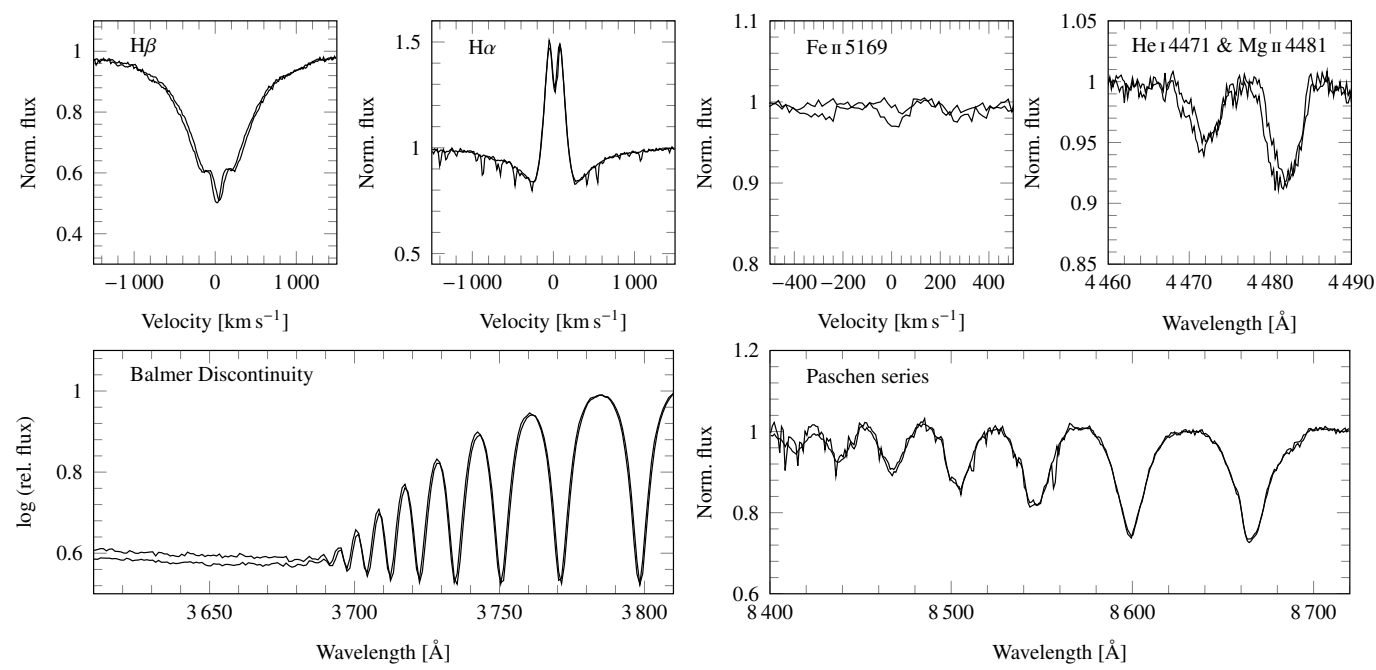

Fig. C.17. Spectrum overview plot for HIP 39183. 

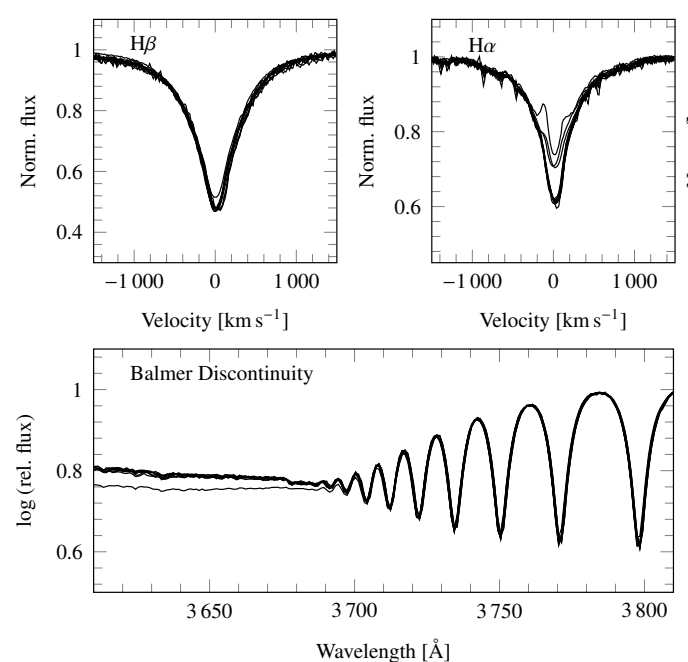

Fig. C.18. Spectrum overview plot for HIP 39483.
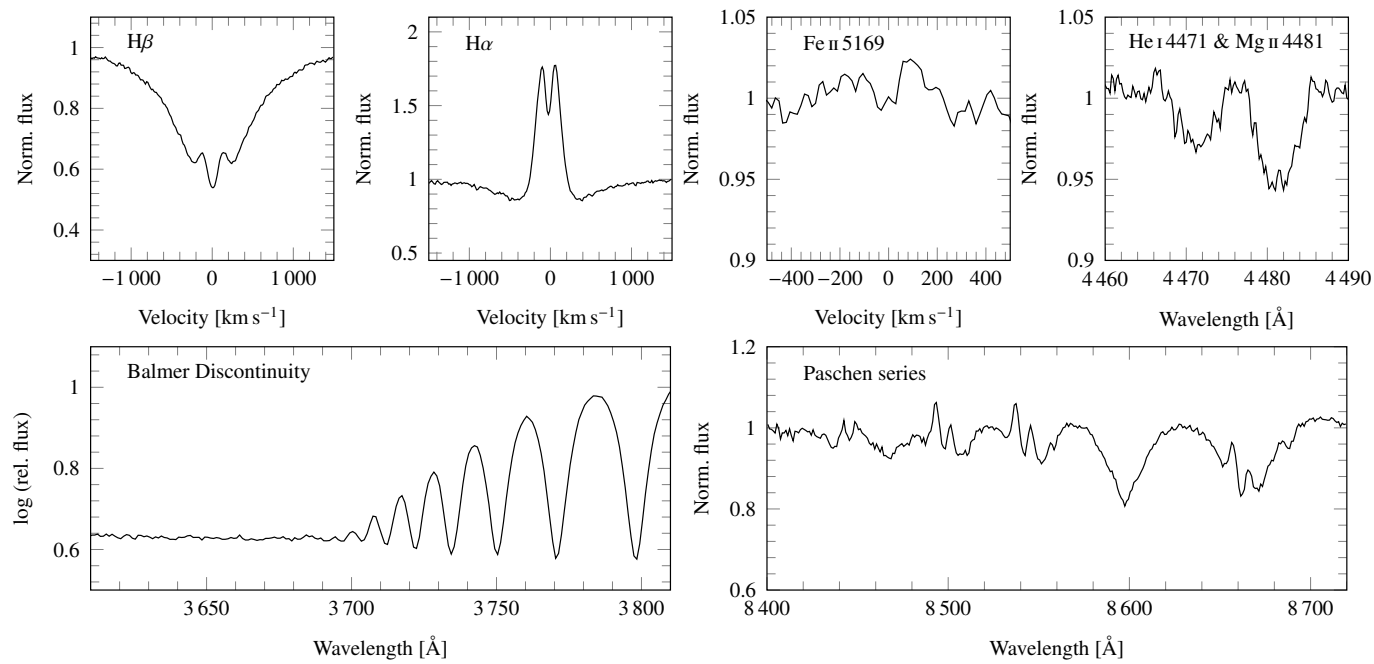
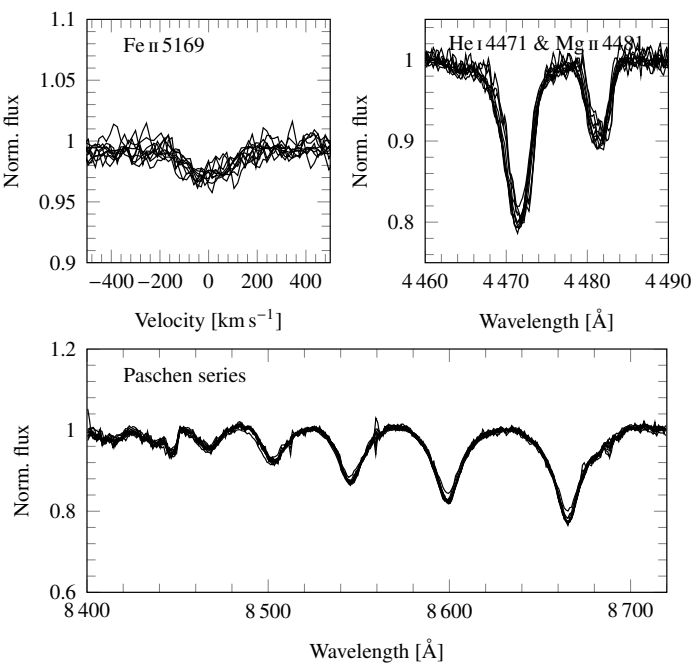

Fig. C.19. Spectrum overview plot for HIP 39595.
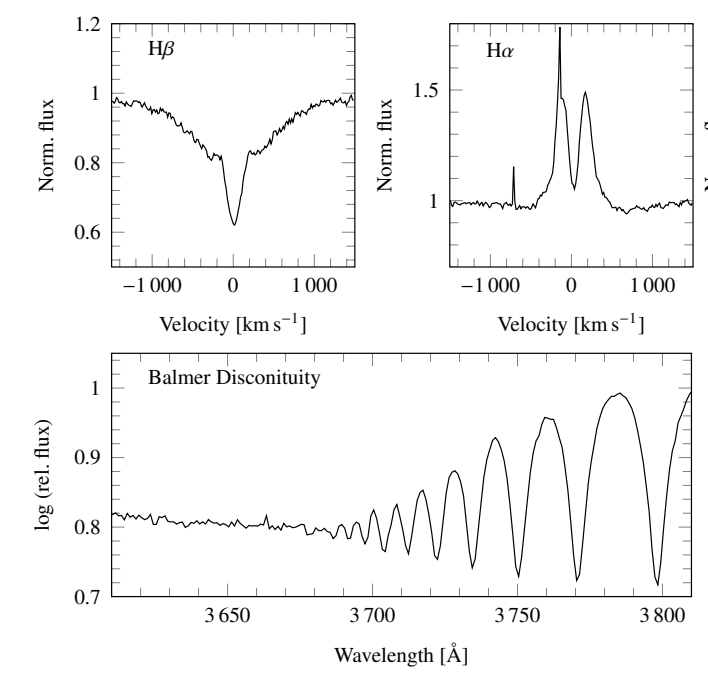
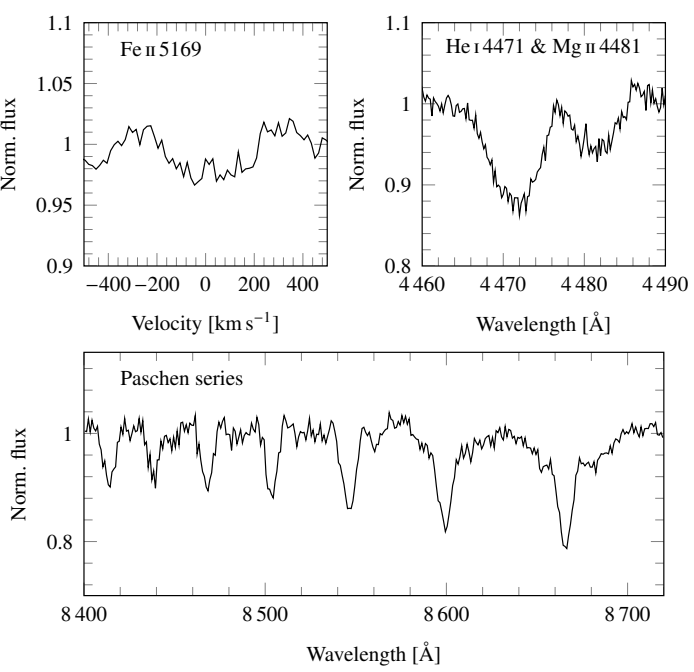

Fig. C.20. Spectrum overview plot for HIP 41085 . 
A. Shokry et al.: Stellar parameters of Be stars observed with X-shooter
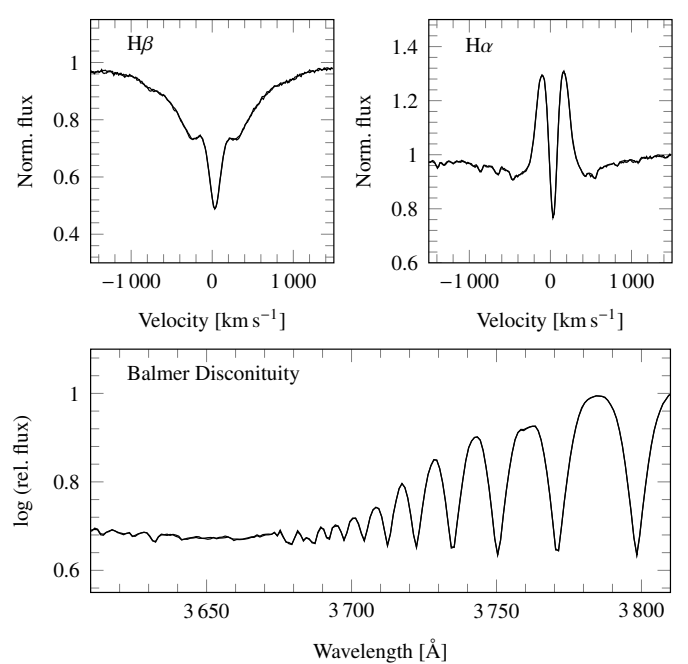

Fig. C.21. Spectrum overview plot for HIP 41268.
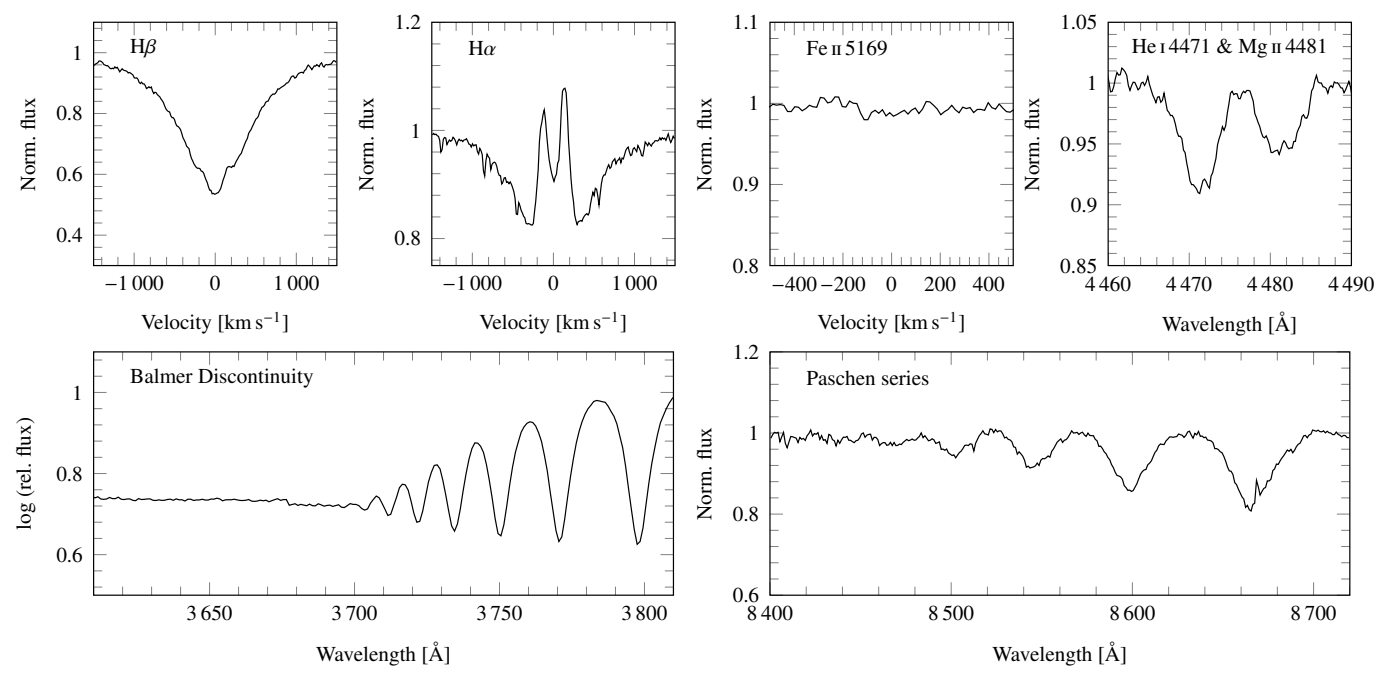
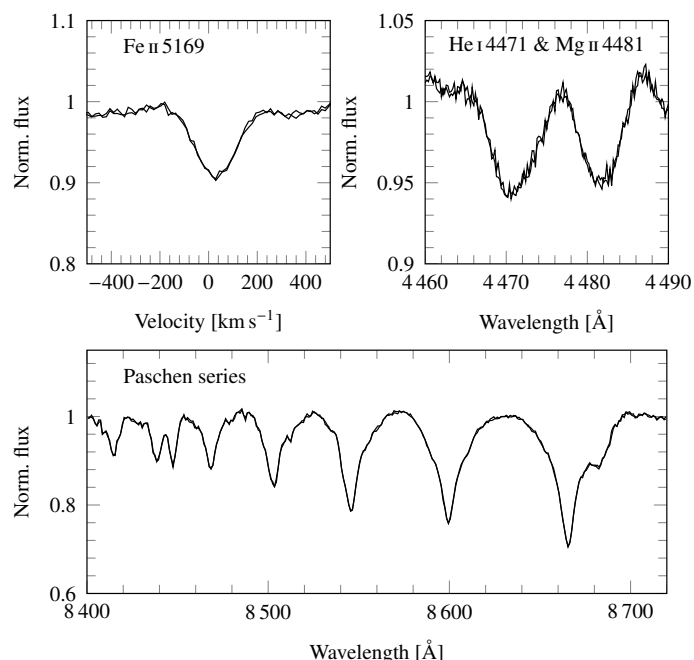

Fig. C.22. Spectrum overview plot for HIP 42060.
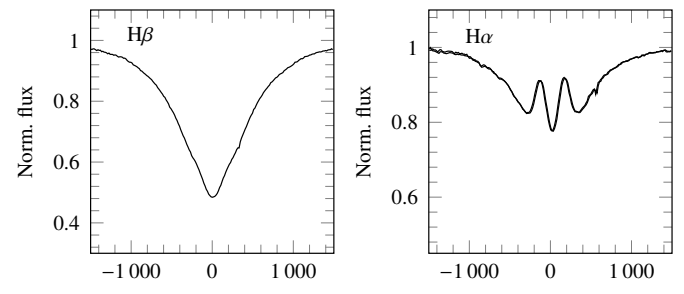

Velocity $\left[\mathrm{km} \mathrm{s}^{-1}\right]$

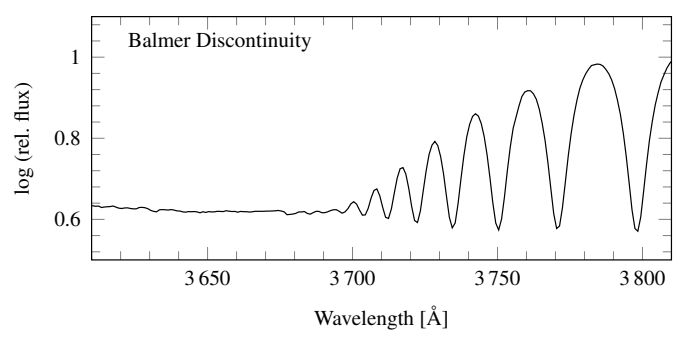


A\&A 609, A108 (2018)
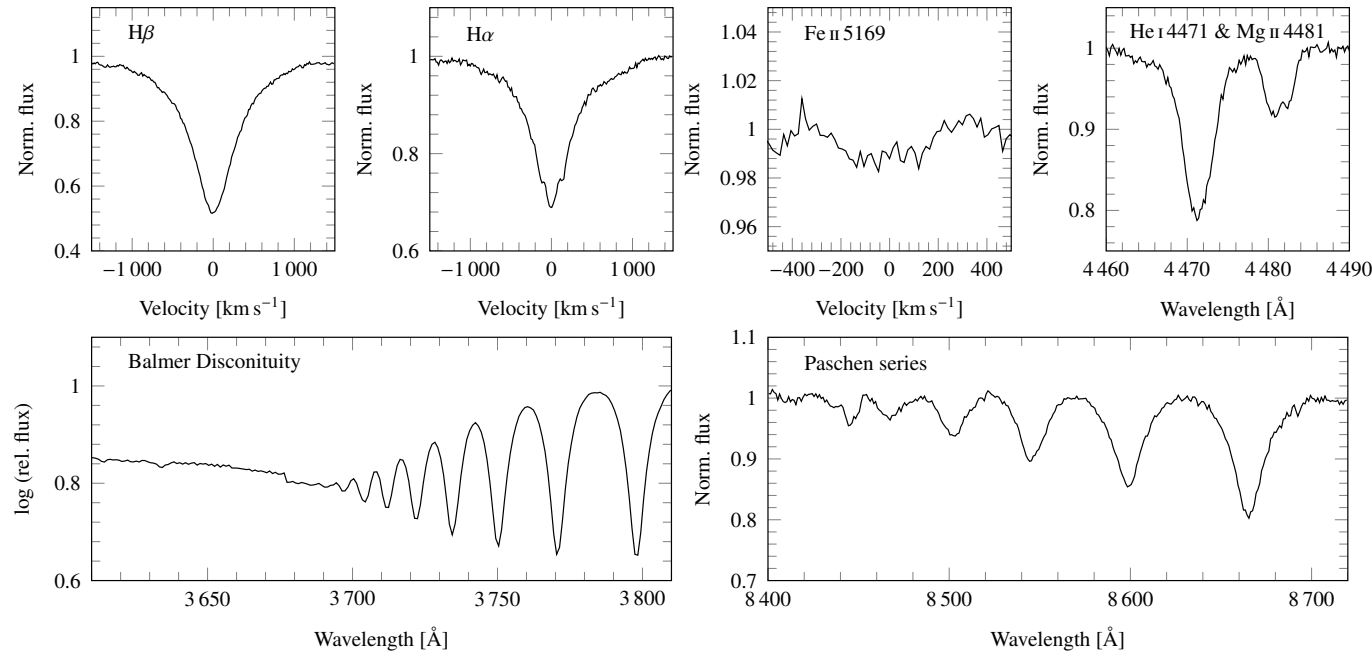

Fig. C.24. Spectrum overview plot for HIP 43114 .
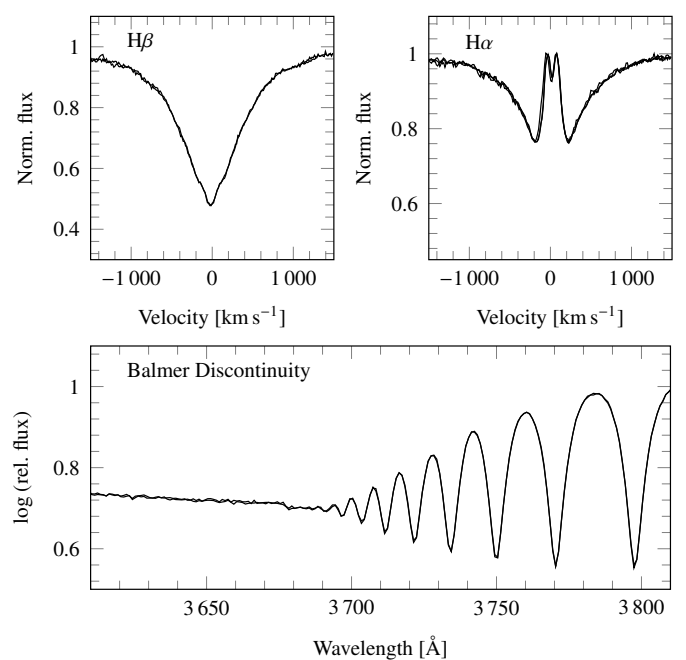

Fig. C.25. Spectrum overview plot for HIP 44423 .
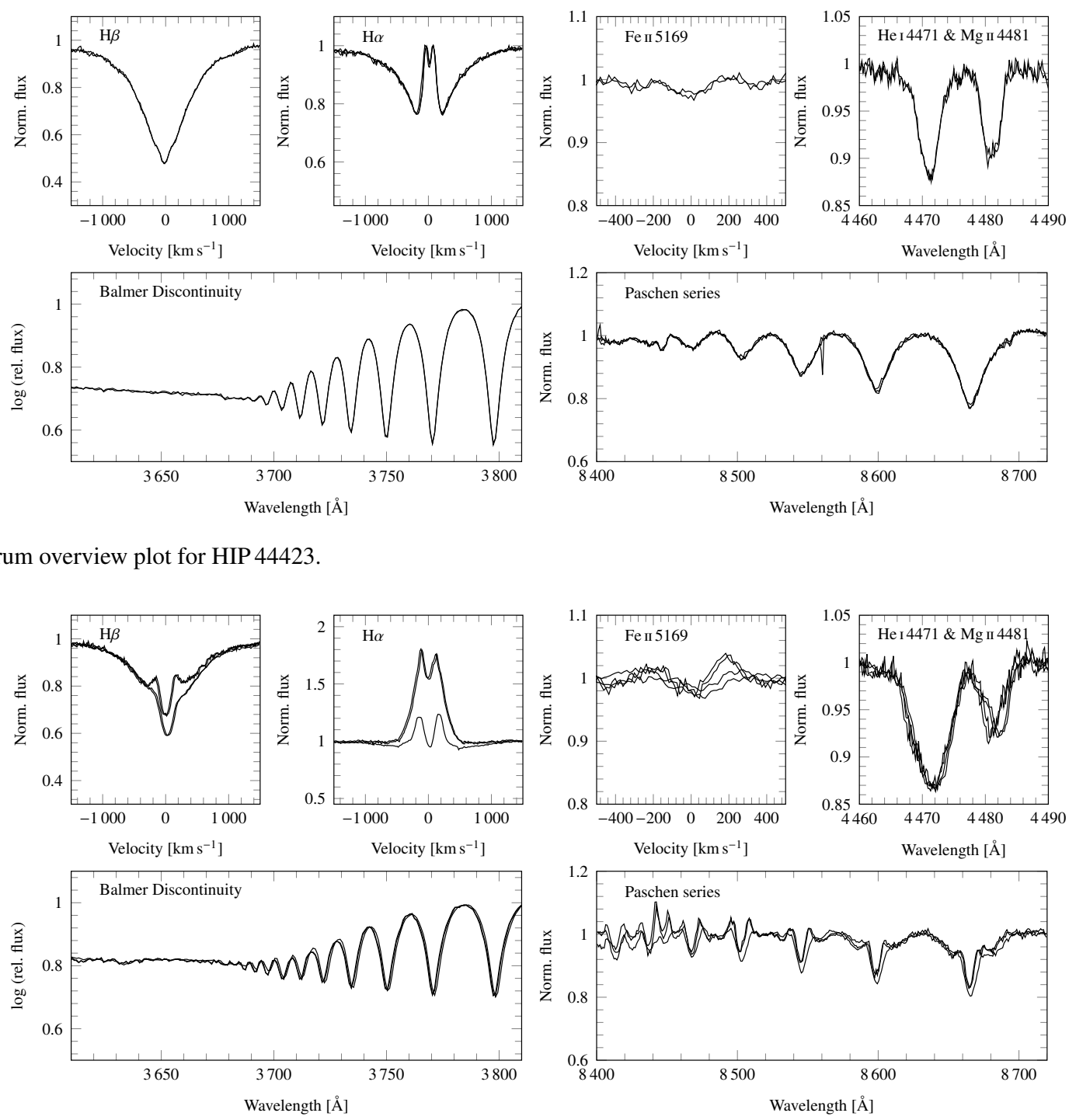

Fig. C.26. Spectrum overview plot for HIP 46329. 
A. Shokry et al.: Stellar parameters of Be stars observed with X-shooter
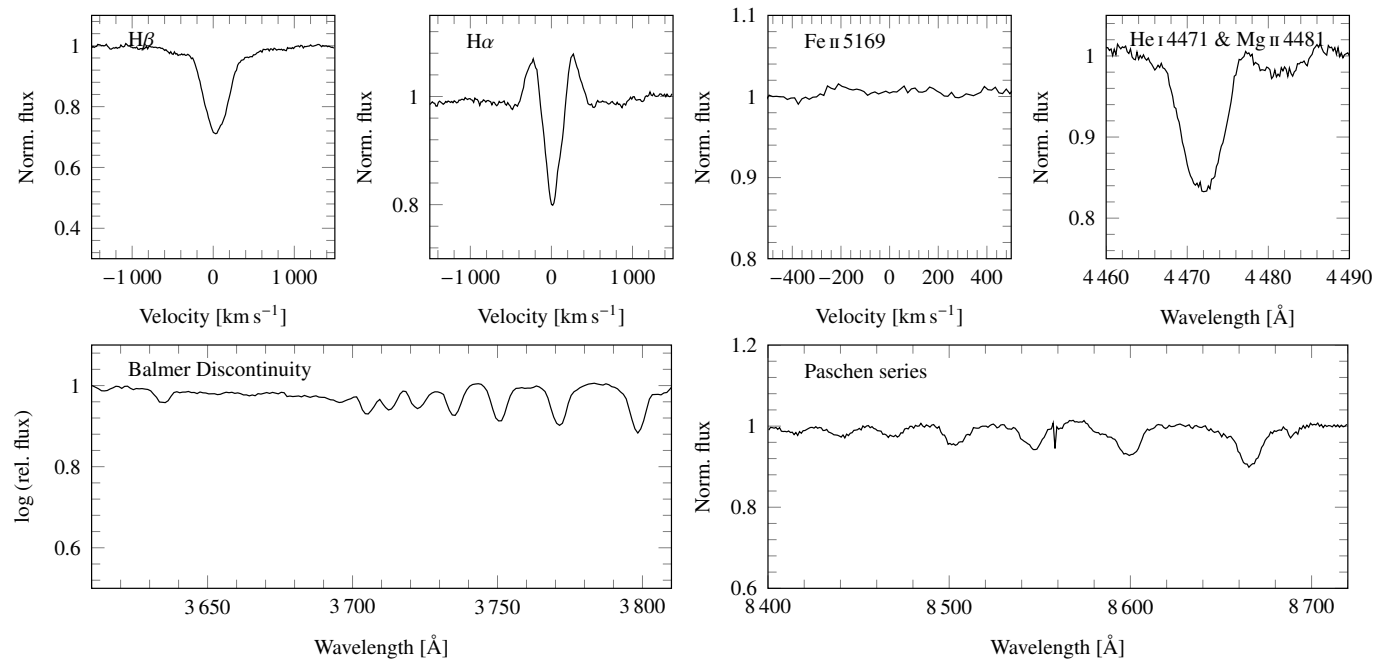

Fig. C.27. Spectrum overview plot for HIP 47868 .
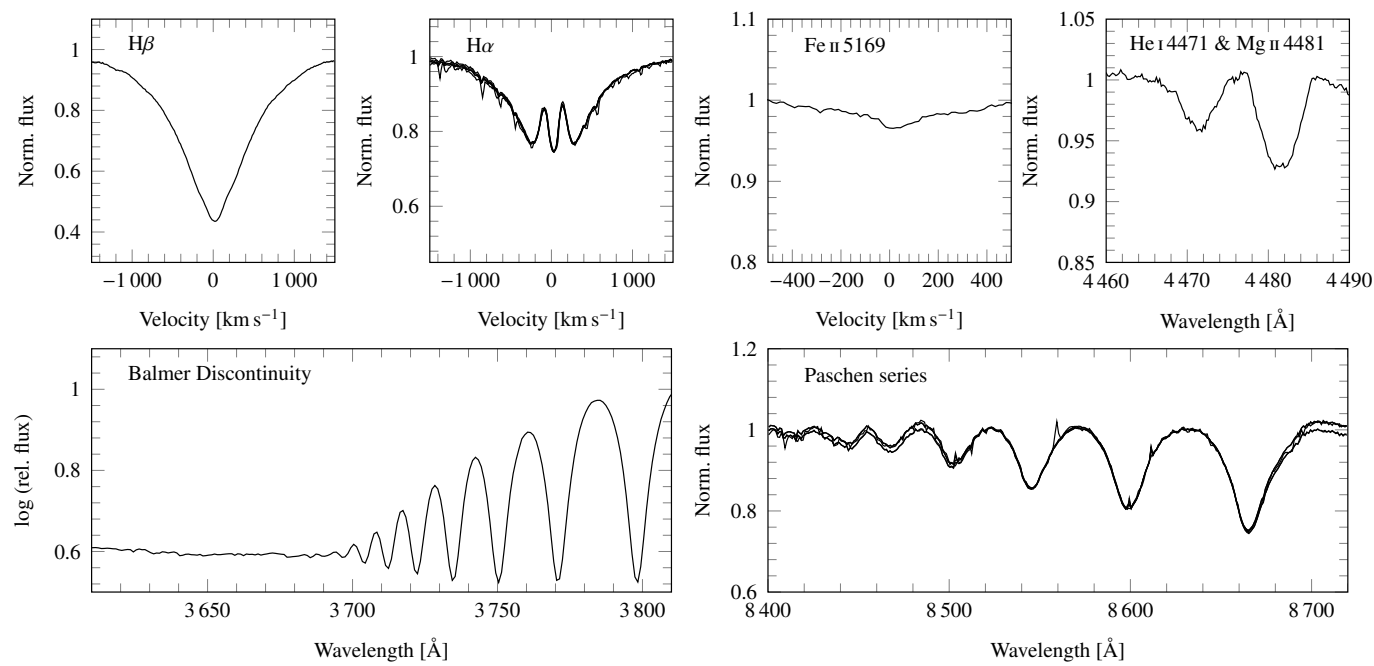

Fig. C.28. Spectrum overview plot for HIP 47962.
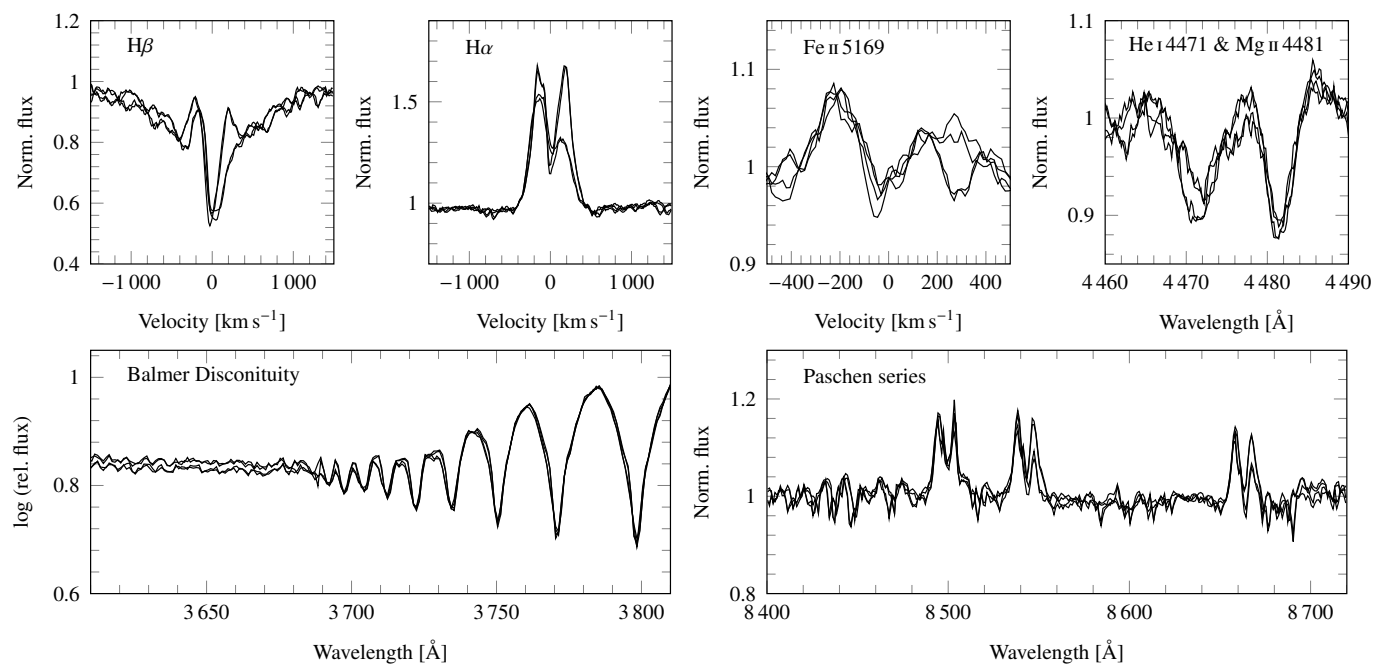

Fig. C.29. Spectrum overview plot for HIP 48582. 

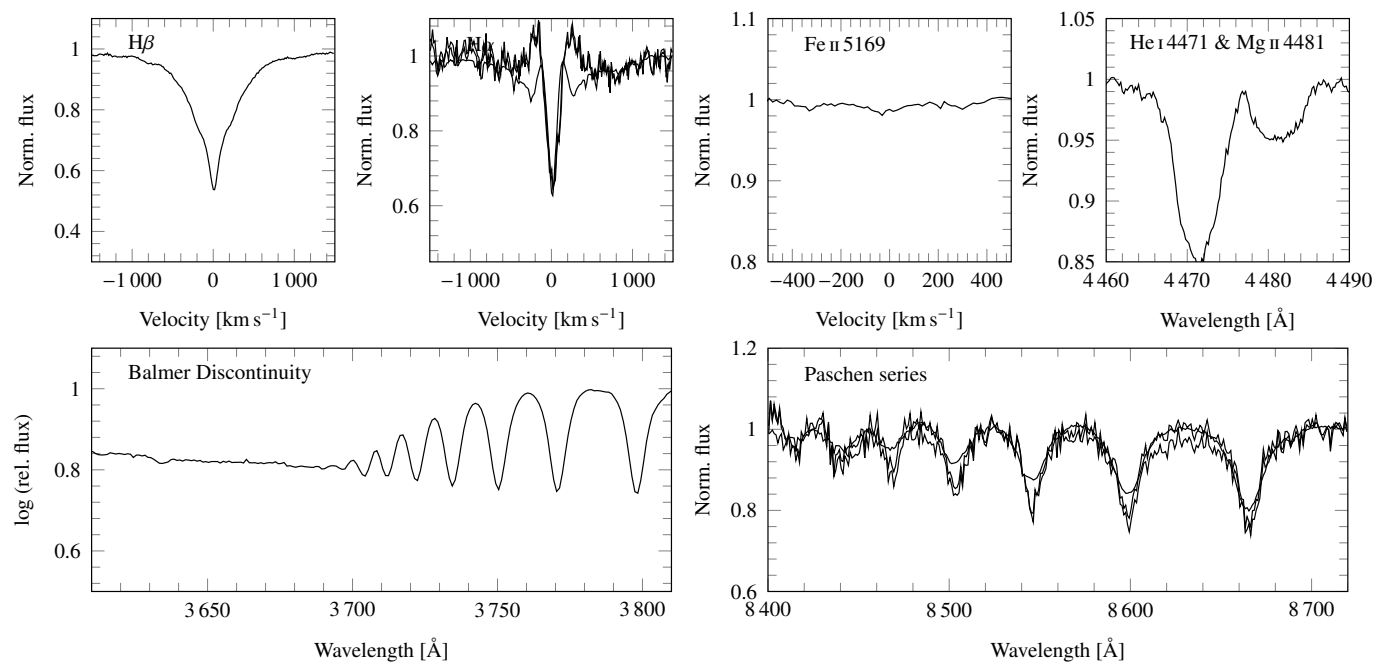

Fig. C.30. Spectrum overview plot for HIP 51444.
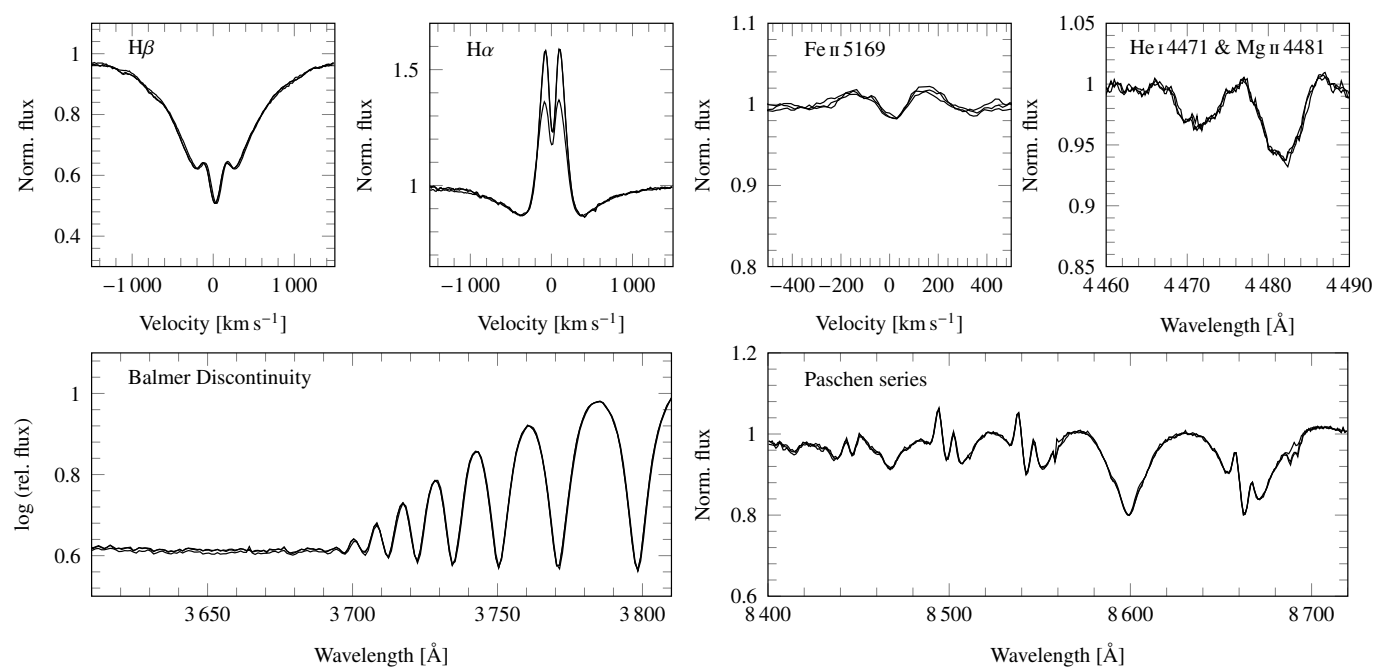

Fig. C.31. Spectrum overview plot for HIP 51491.
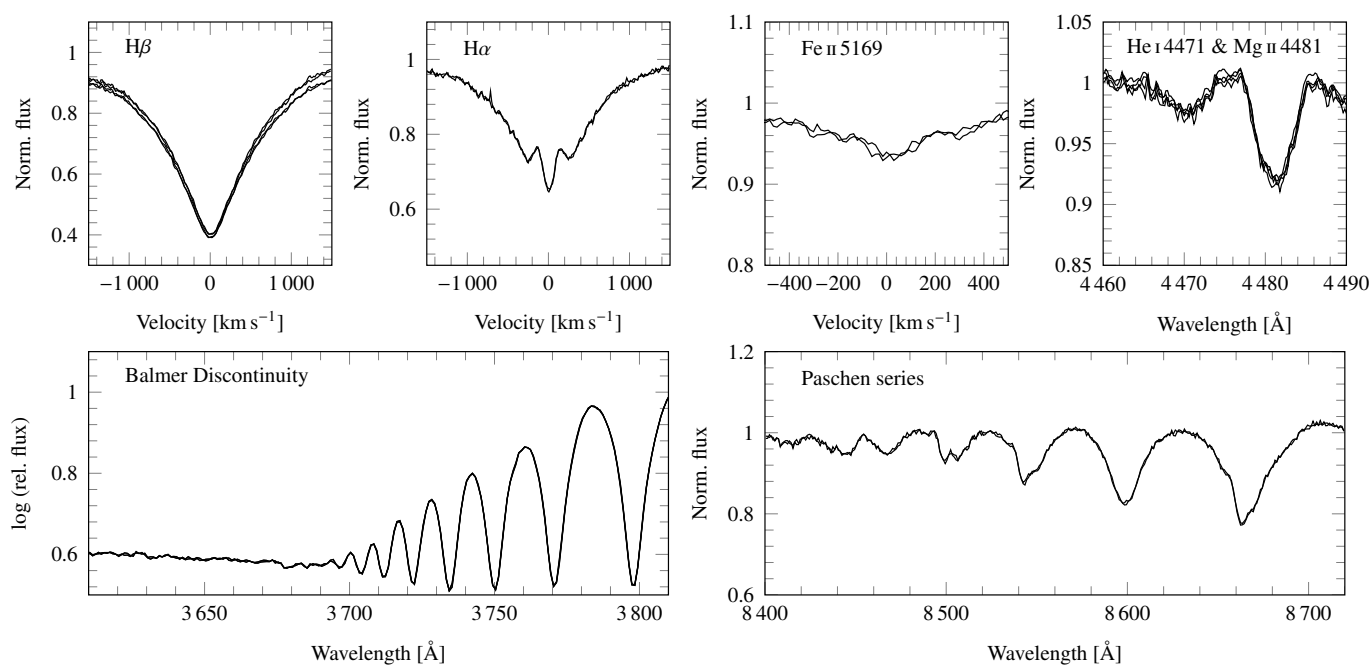

Fig. C.32. Spectrum overview plot for HIP 51546. 
A. Shokry et al.: Stellar parameters of Be stars observed with X-shooter
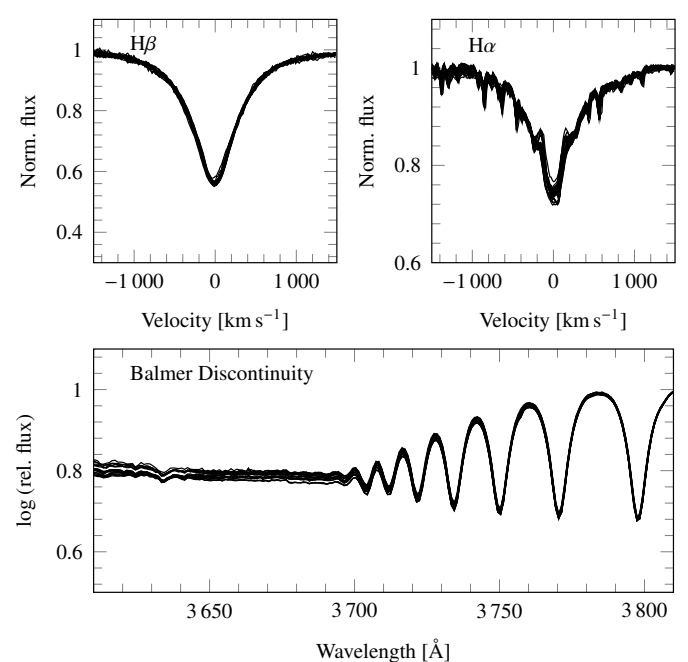

Fig. C.33. Spectrum overview plot for HIP 52977.
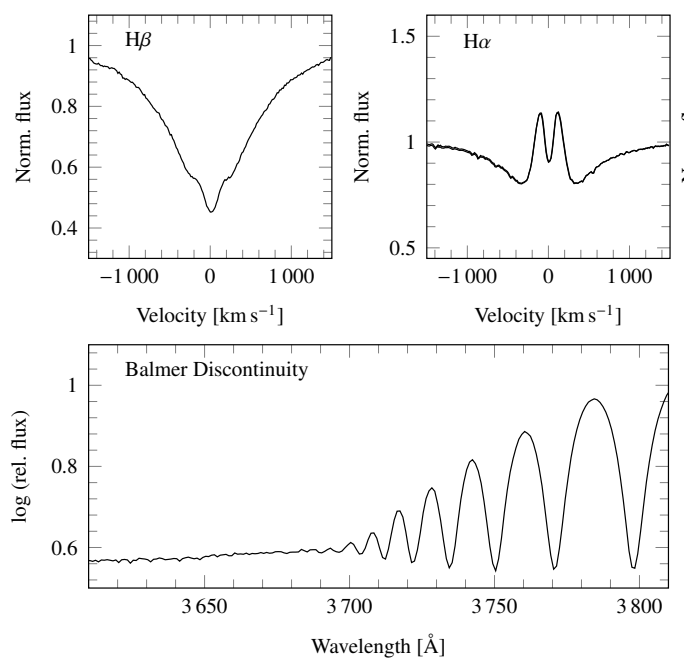

Fig. C.34. Spectrum overview plot for HIP 56393.
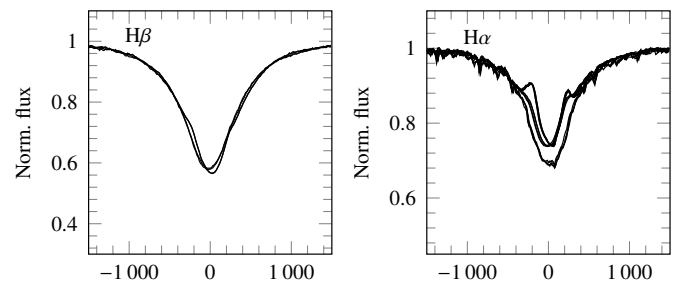

Velocity $\left[\mathrm{km} \mathrm{s}^{-1}\right]$
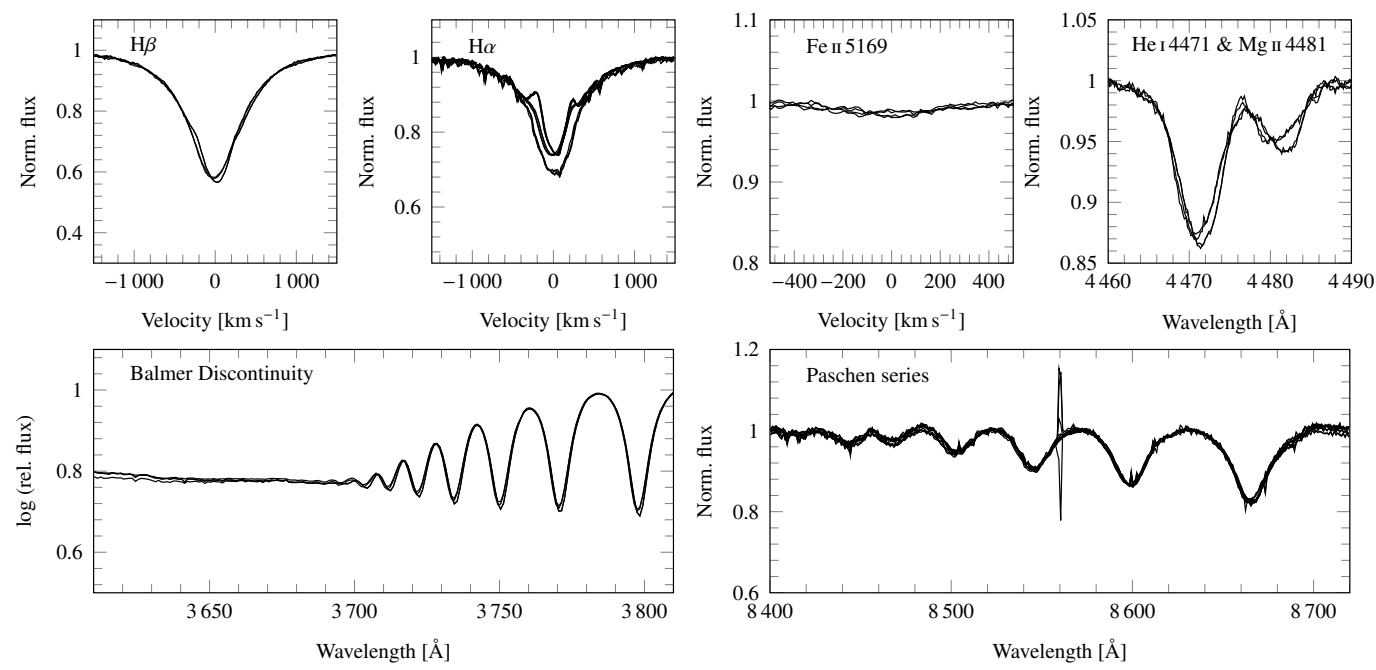

Fig. C.35. Spectrum overview plot for HIP 57861. 

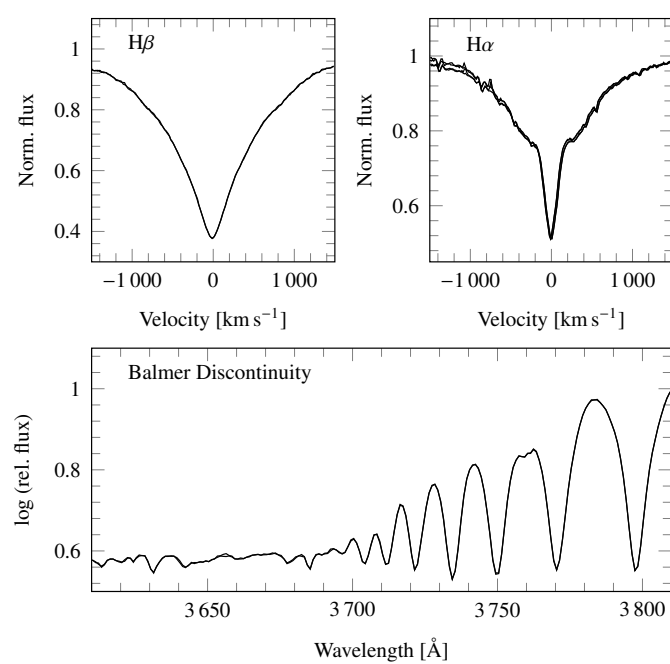

Fig. C.36. Spectrum overview plot for HIP 59970.
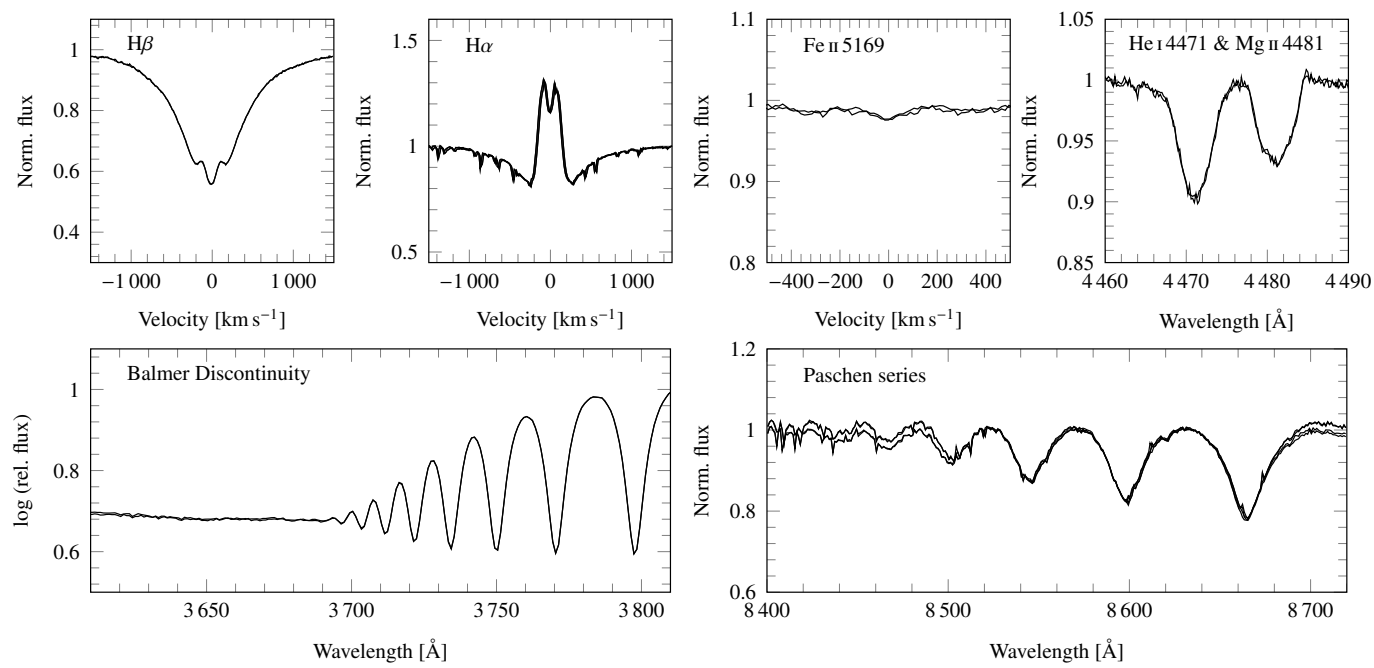

Fig. C.37. Spectrum overview plot for HIP 64501.
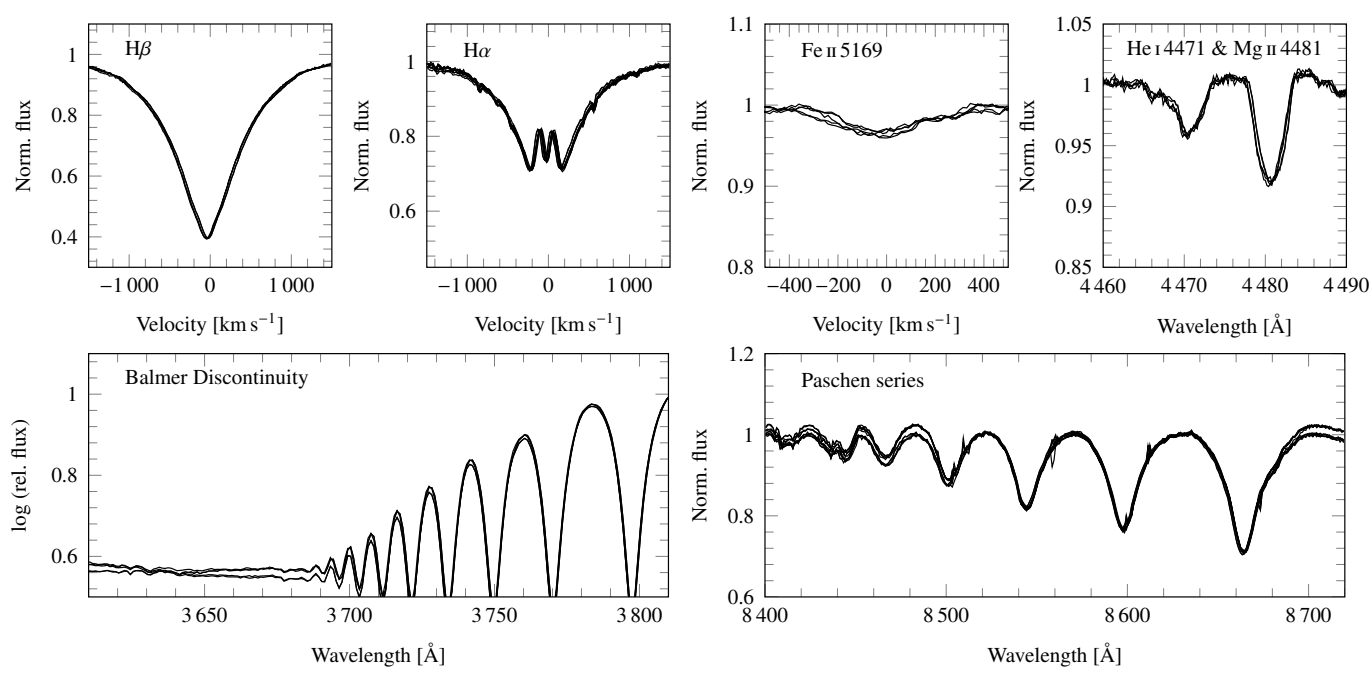

Fig. C.38. Spectrum overview plot for HIP 64867. 
A. Shokry et al.: Stellar parameters of Be stars observed with X-shooter
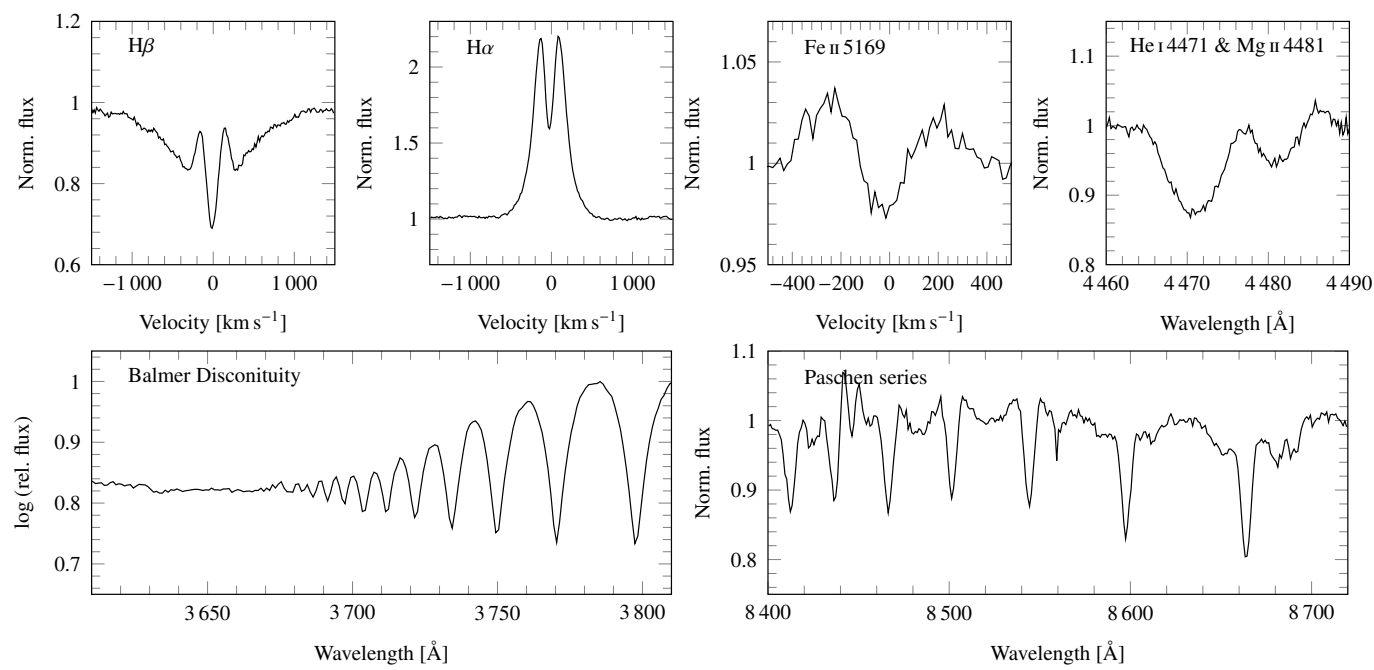

Fig. C.39. Spectrum overview plot for HIP 66339.
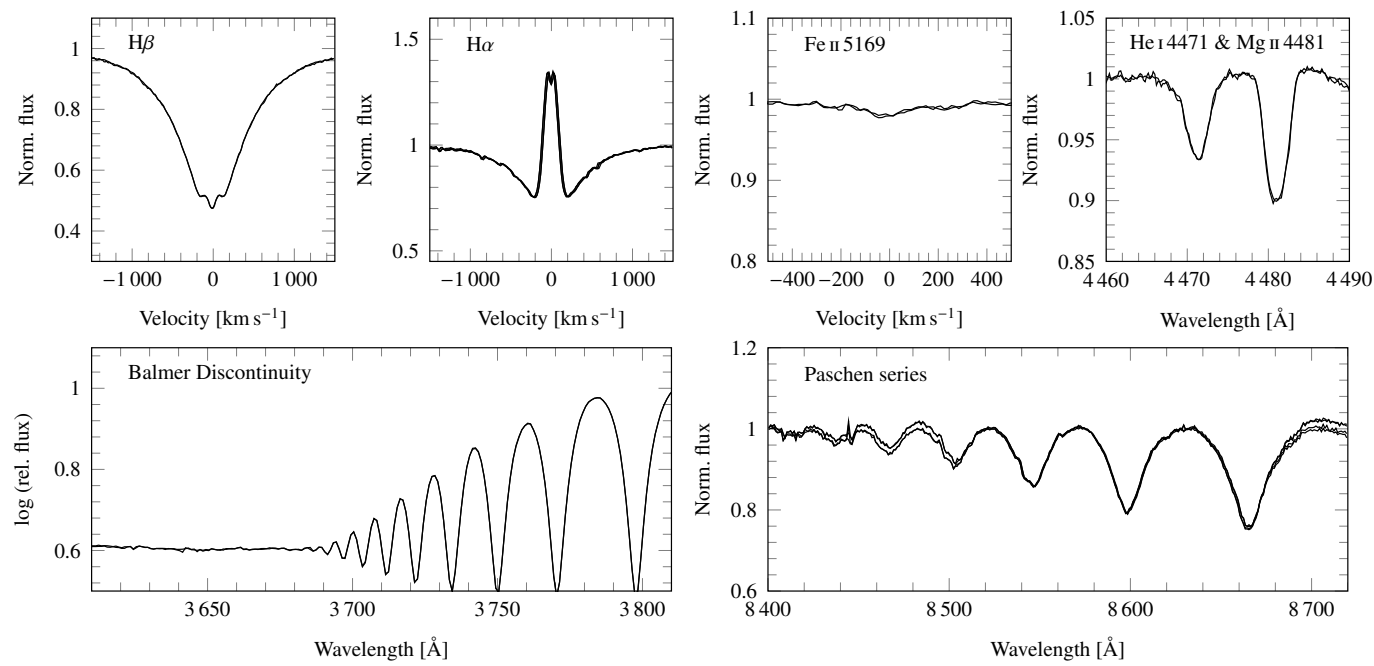

Fig. C.40. Spectrum overview plot for HIP 66351.
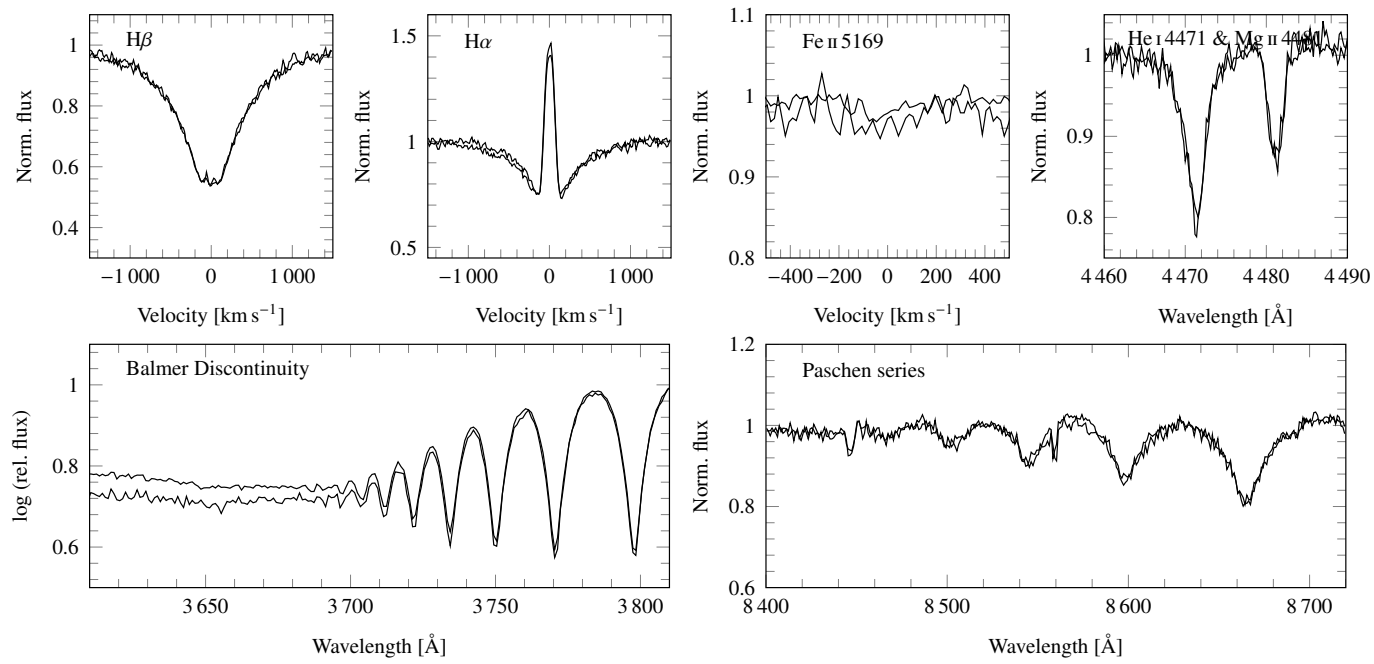

Fig. C.41. Spectrum overview plot for HIP 68100. 

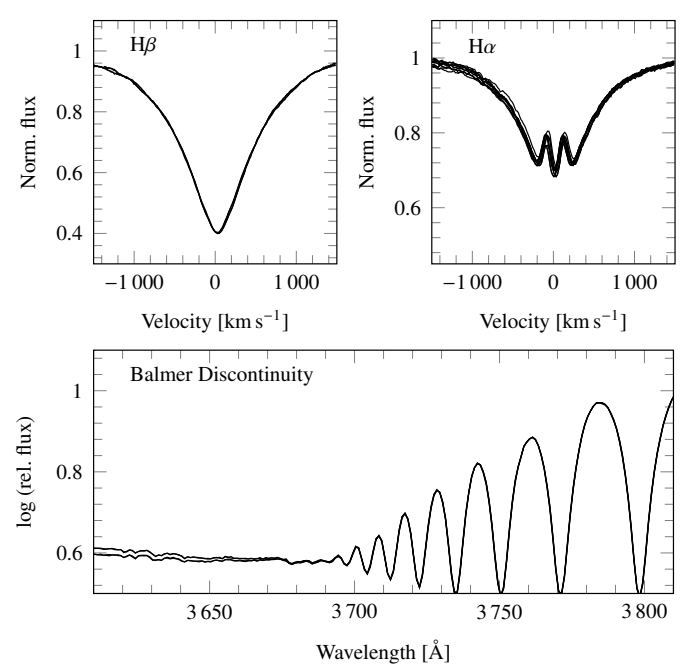

Fig. C.42. Spectrum overview plot for HIP 69429.
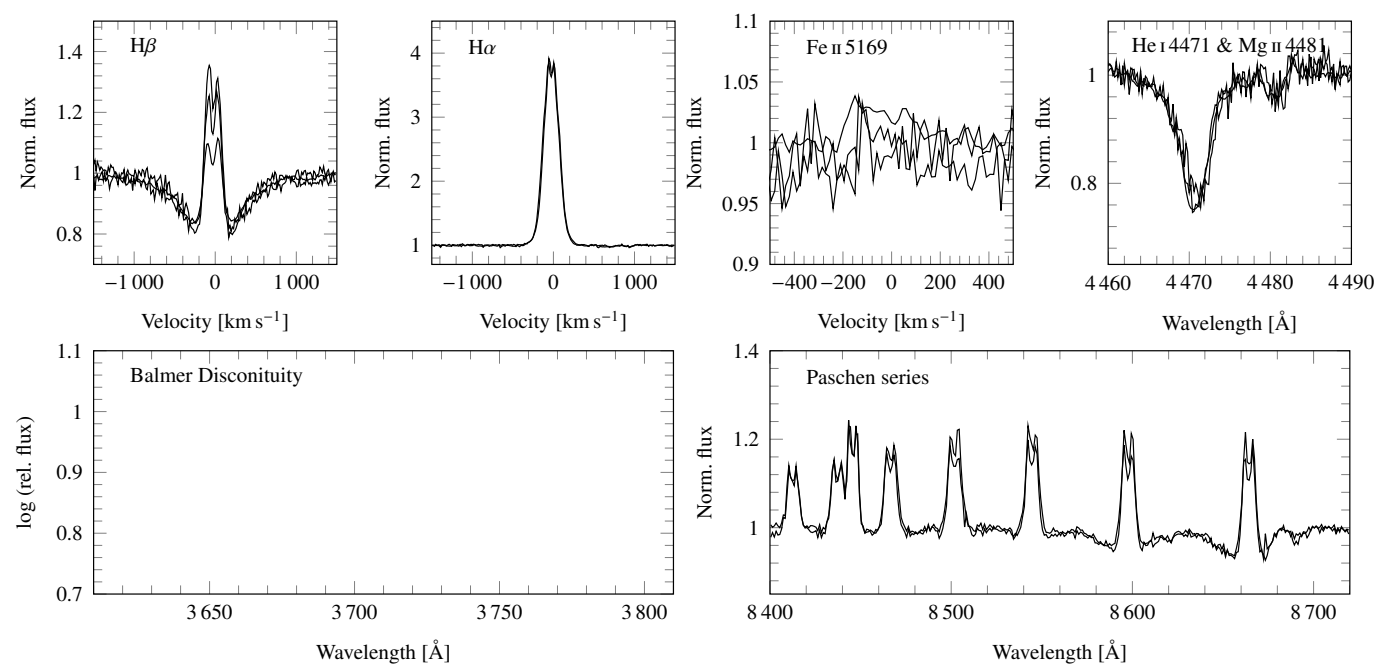

Fig. C.43. Spectrum overview plot for HIP 71668.
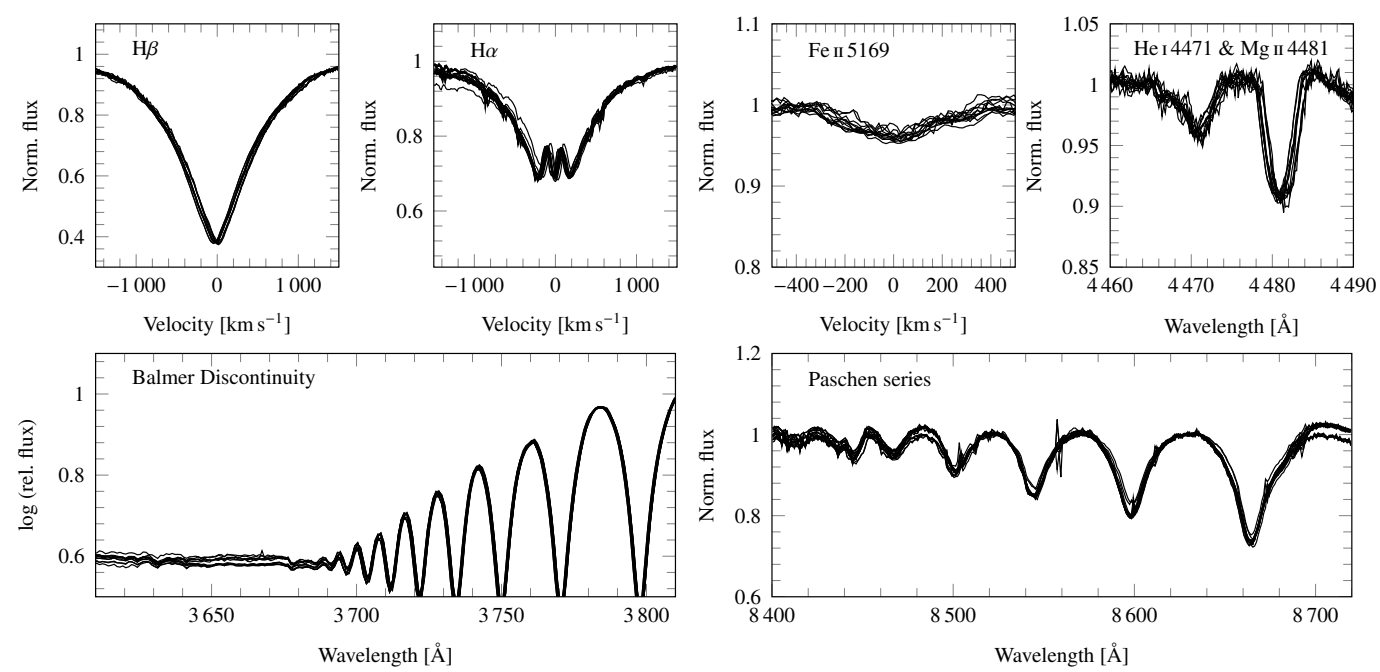

Fig. C.44. Spectrum overview plot for HIP 71974. 
A. Shokry et al.: Stellar parameters of Be stars observed with X-shooter
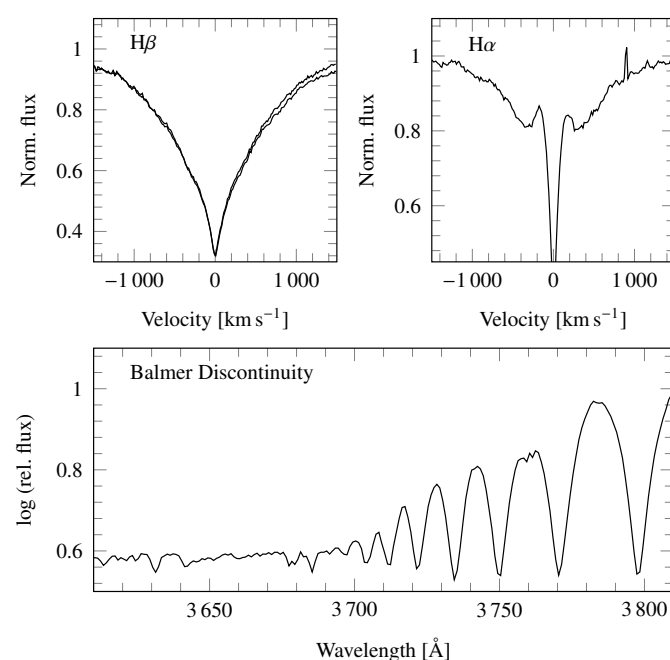

Fig. C.45. Spectrum overview plot for HIP 78375.
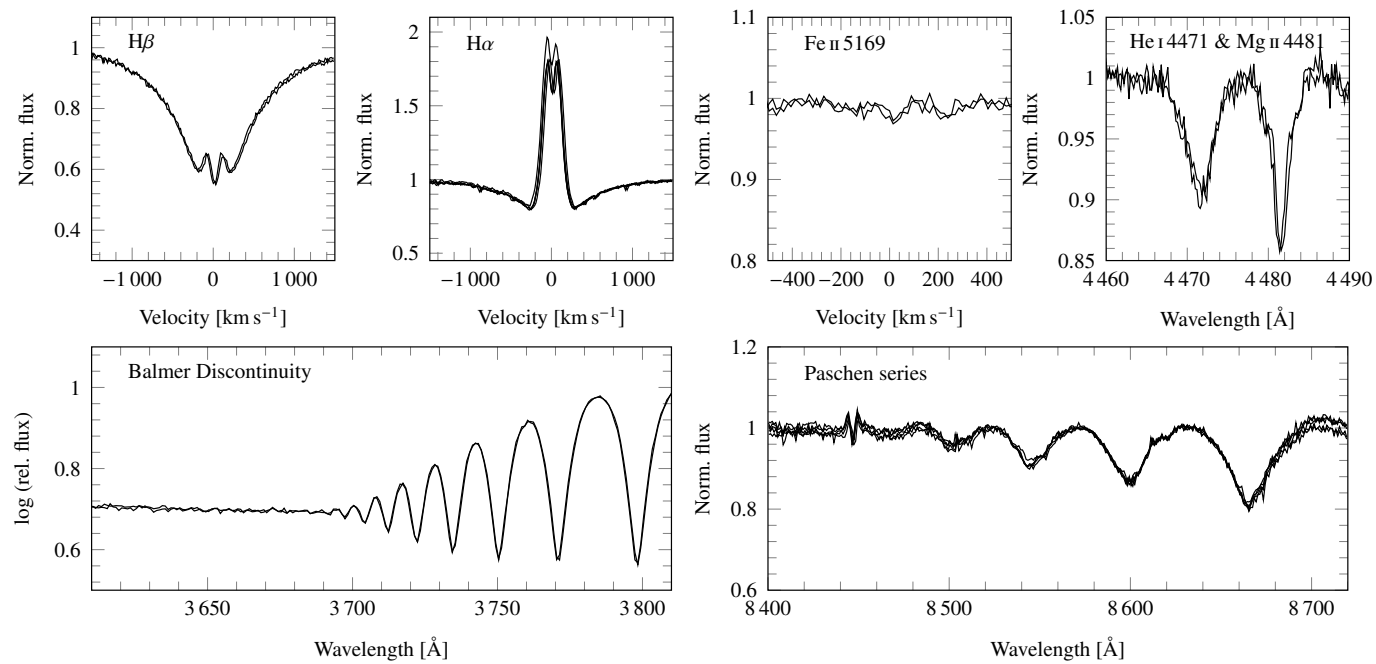

Fig. C.46. Spectrum overview plot for HIP 80577.
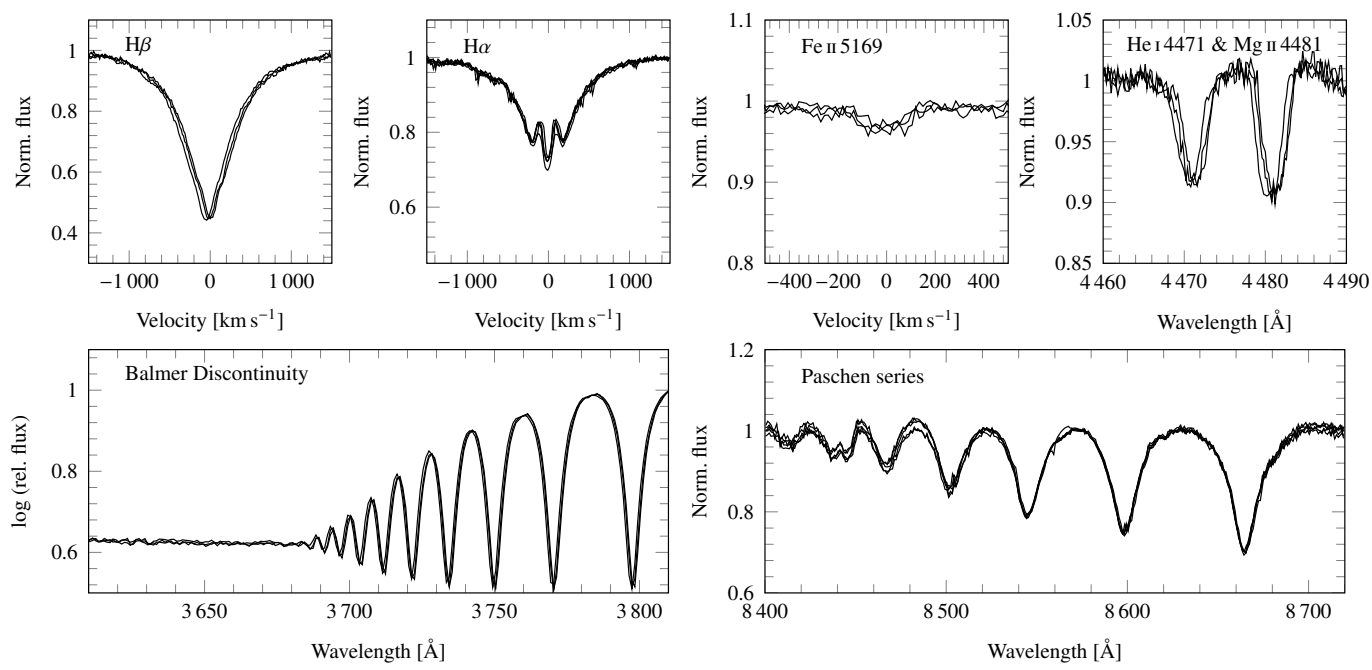

Fig. C.47. Spectrum overview plot for HIP 80820. 

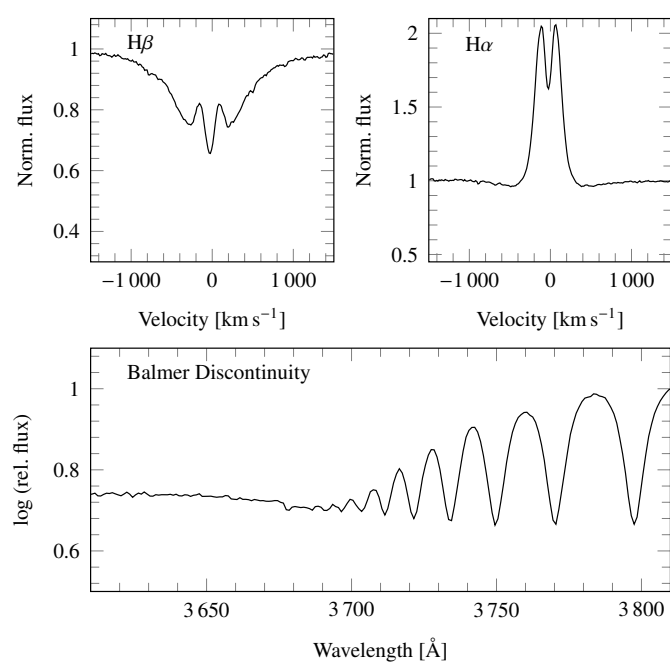

Fig. C.48. Spectrum overview plot for HIP 82874 .
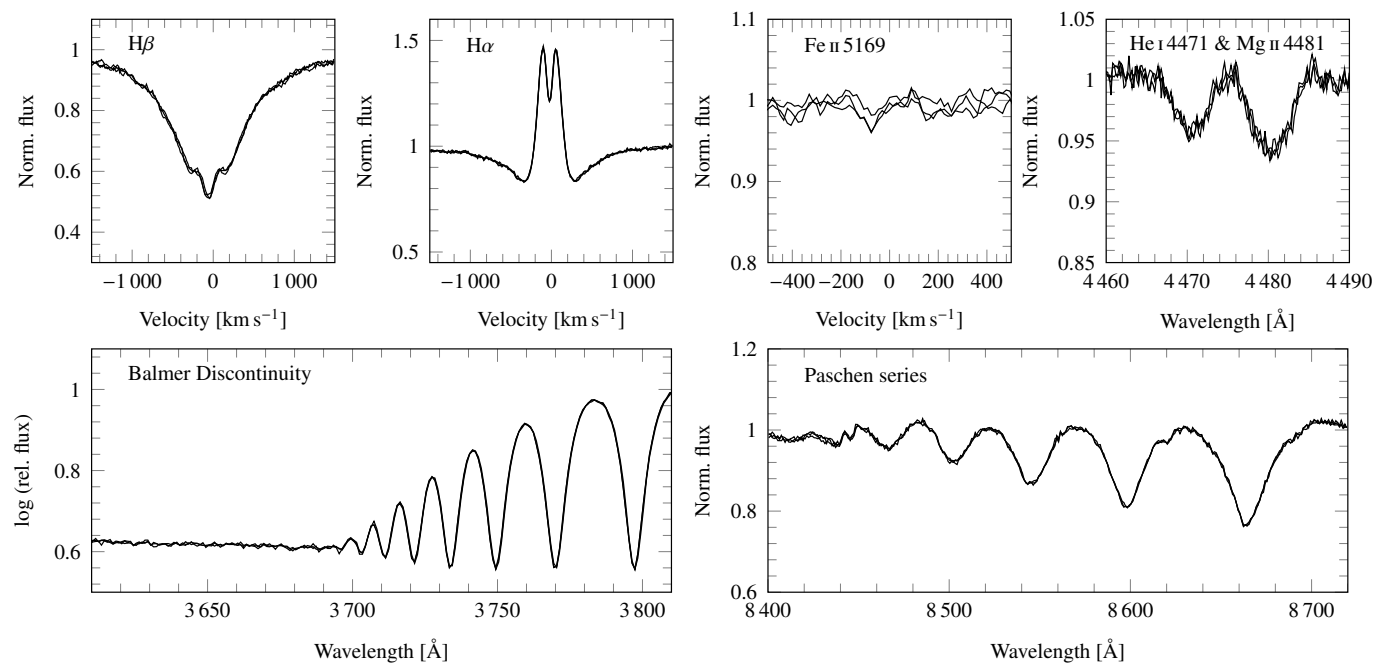

Fig. C.49. Spectrum overview plot for HIP 83278 .
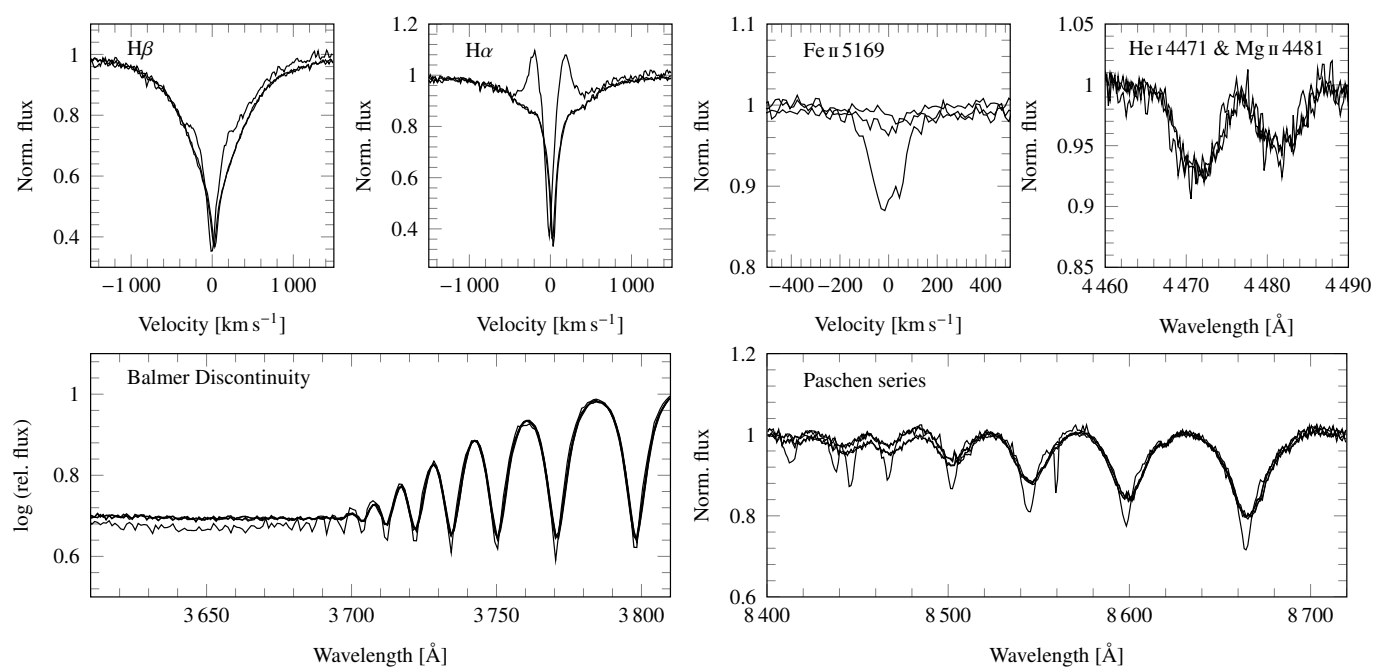

Fig. C.50. Spectrum overview plot for HIP 84184 . 
A. Shokry et al.: Stellar parameters of Be stars observed with X-shooter
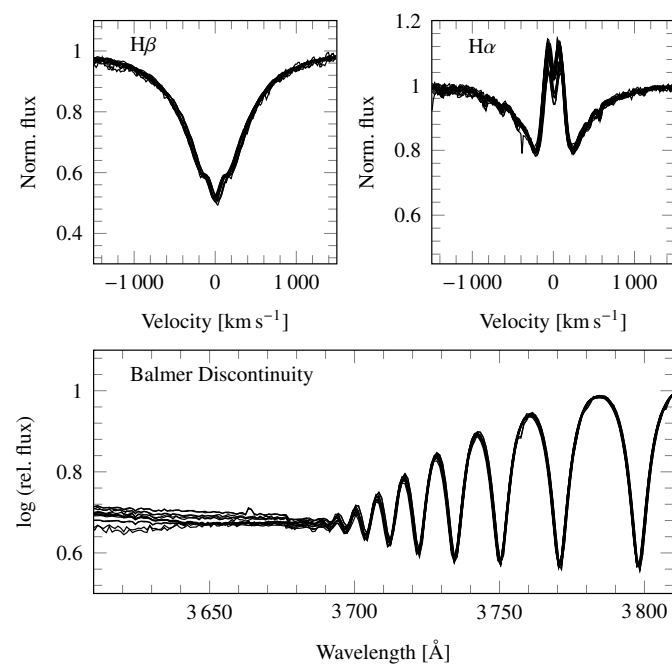

Fig. C.51. Spectrum overview plot for HIP 85138 .
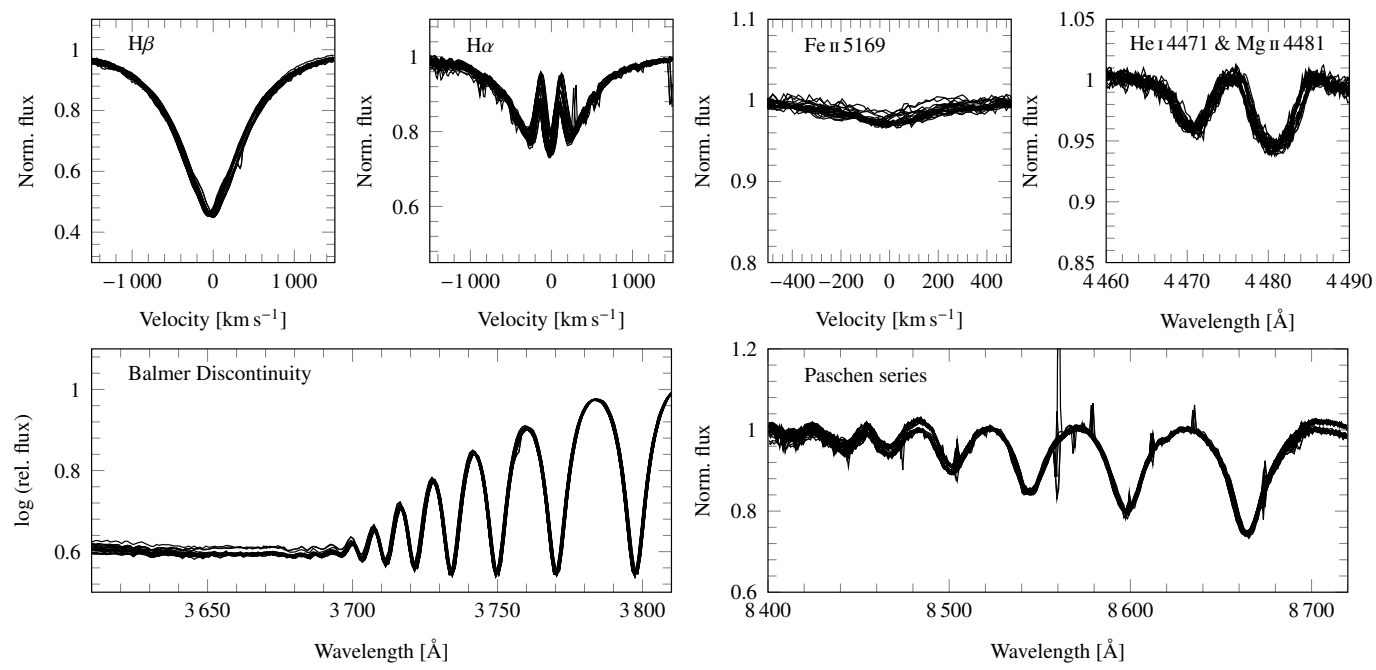
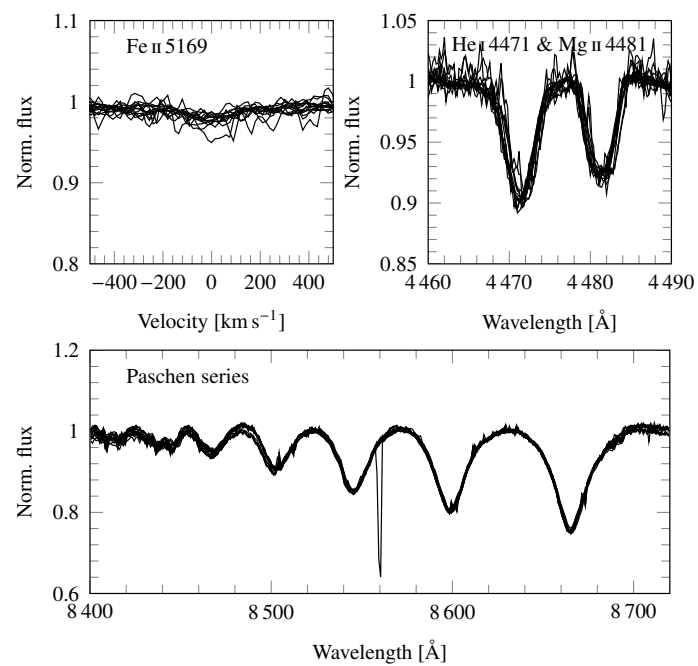

Fig. C.52. Spectrum overview plot for HIP 85195.
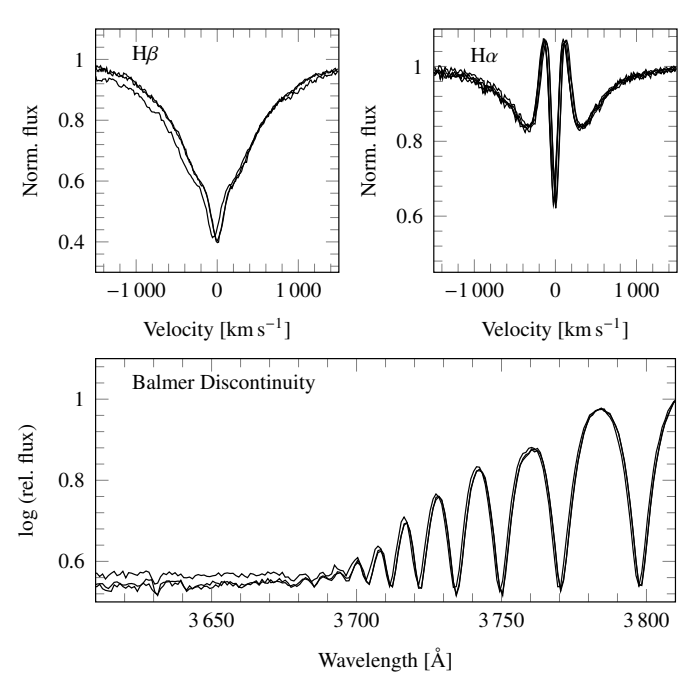
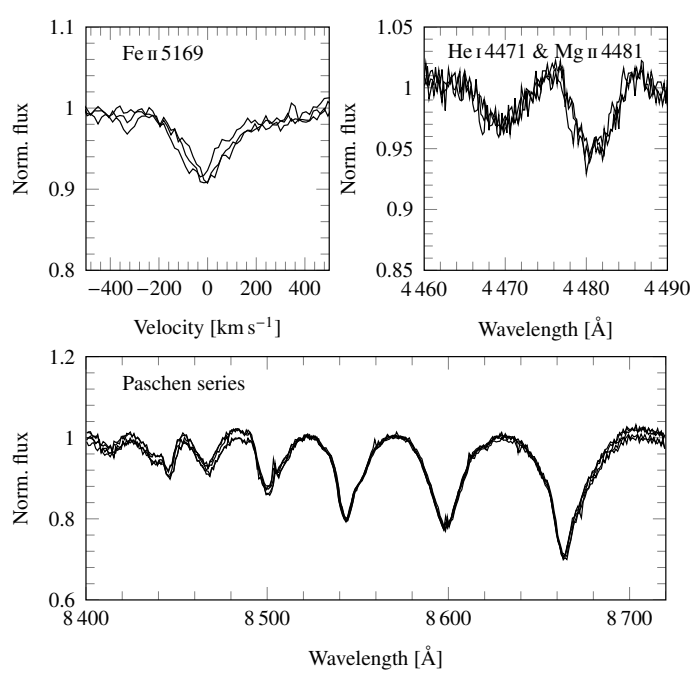

Fig. C.53. Spectrum overview plot for HIP 85566. 

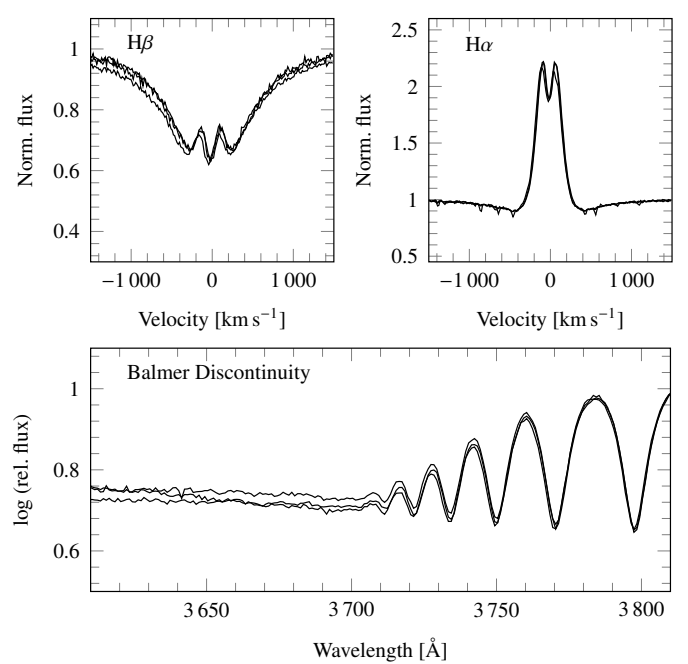

Fig. C.54. Spectrum overview plot for HIP 87032.
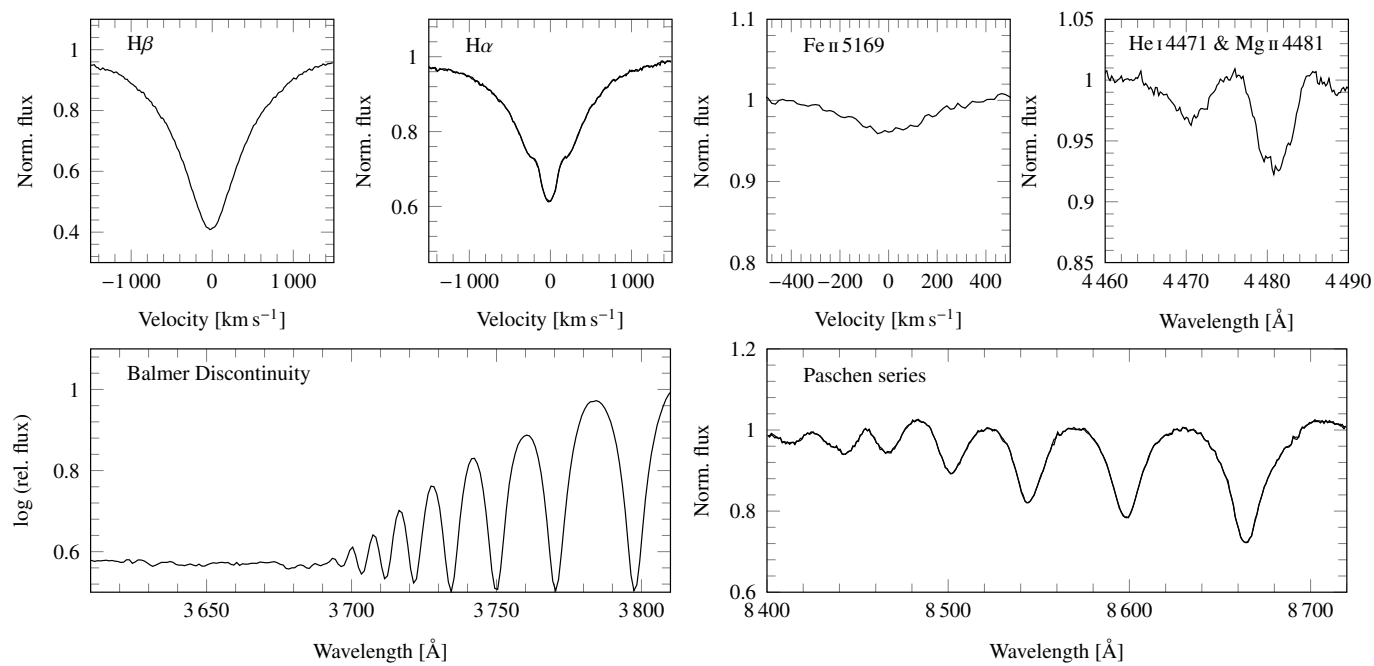

Fig. C.55. Spectrum overview plot for HIP 87698 .
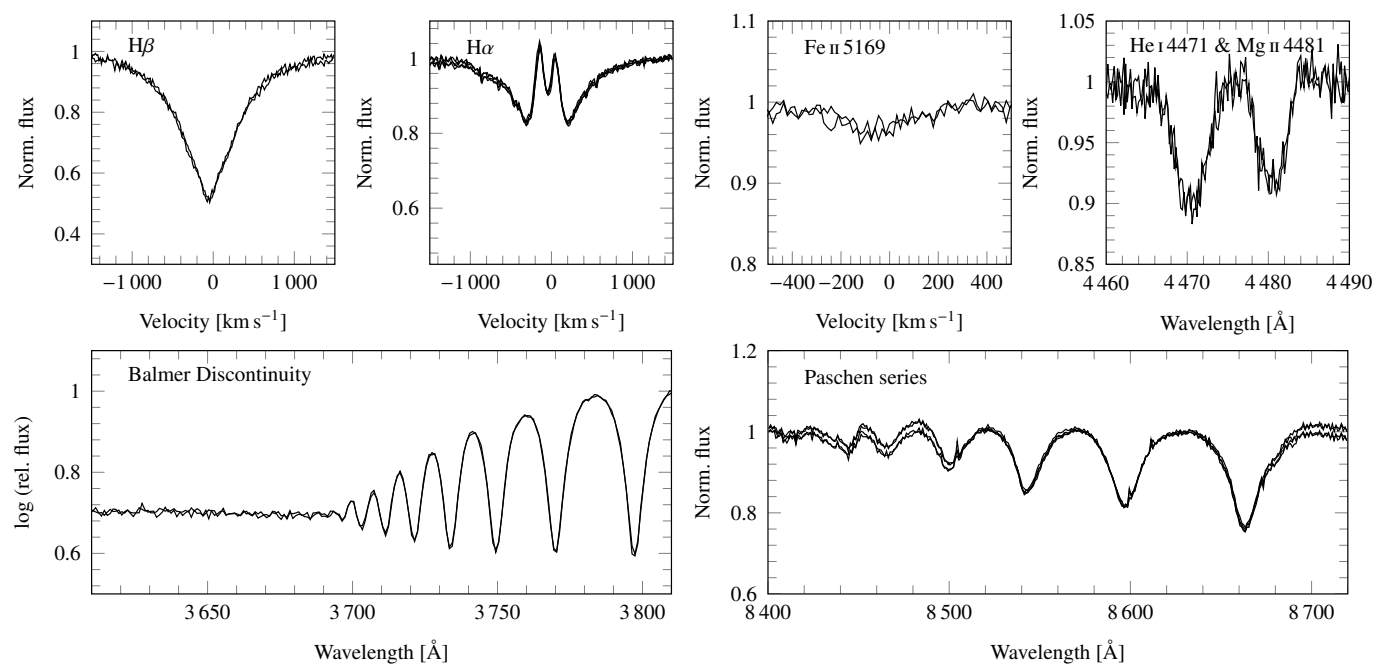

Fig. C.56. Spectrum overview plot for HIP 88172. 
A. Shokry et al.: Stellar parameters of Be stars observed with X-shooter
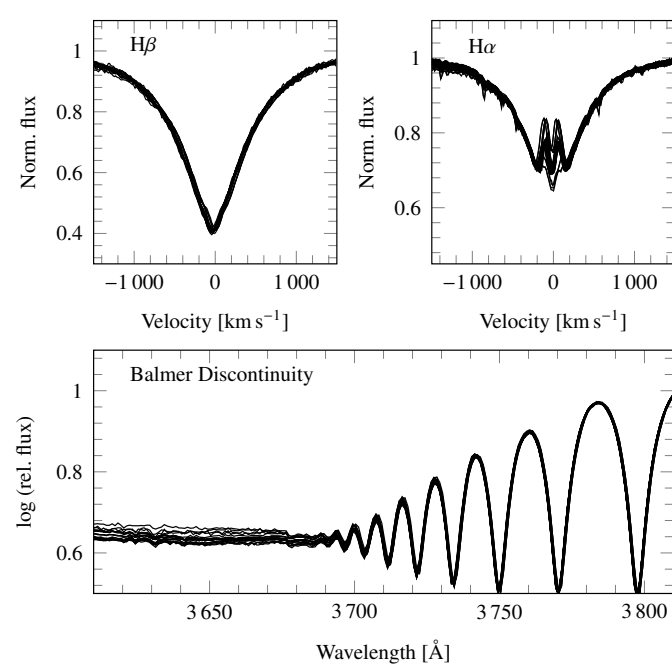
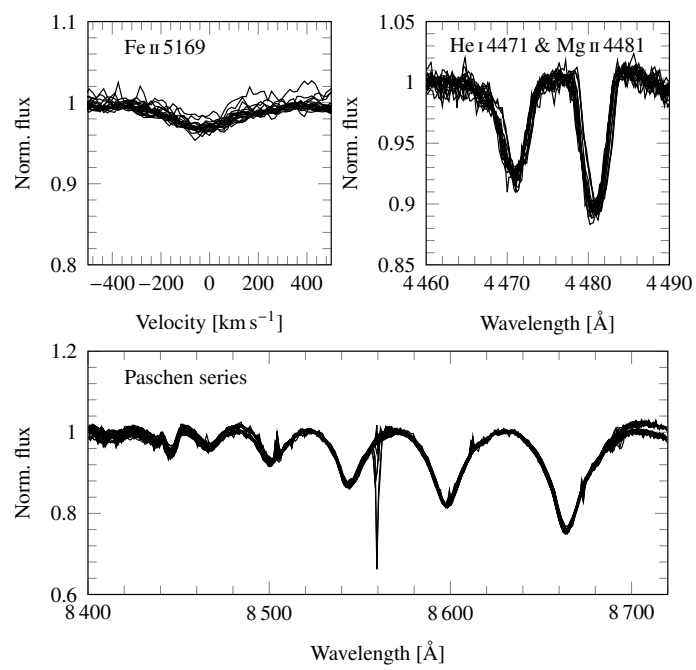

Fig. C.57. Spectrum overview plot for HIP 88374.
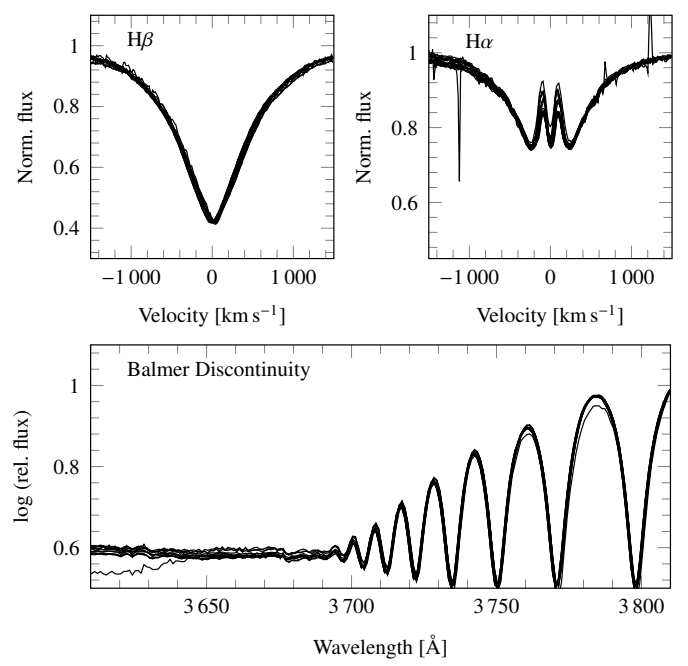

Fig. C.58. Spectrum overview plot for HIP 89486.
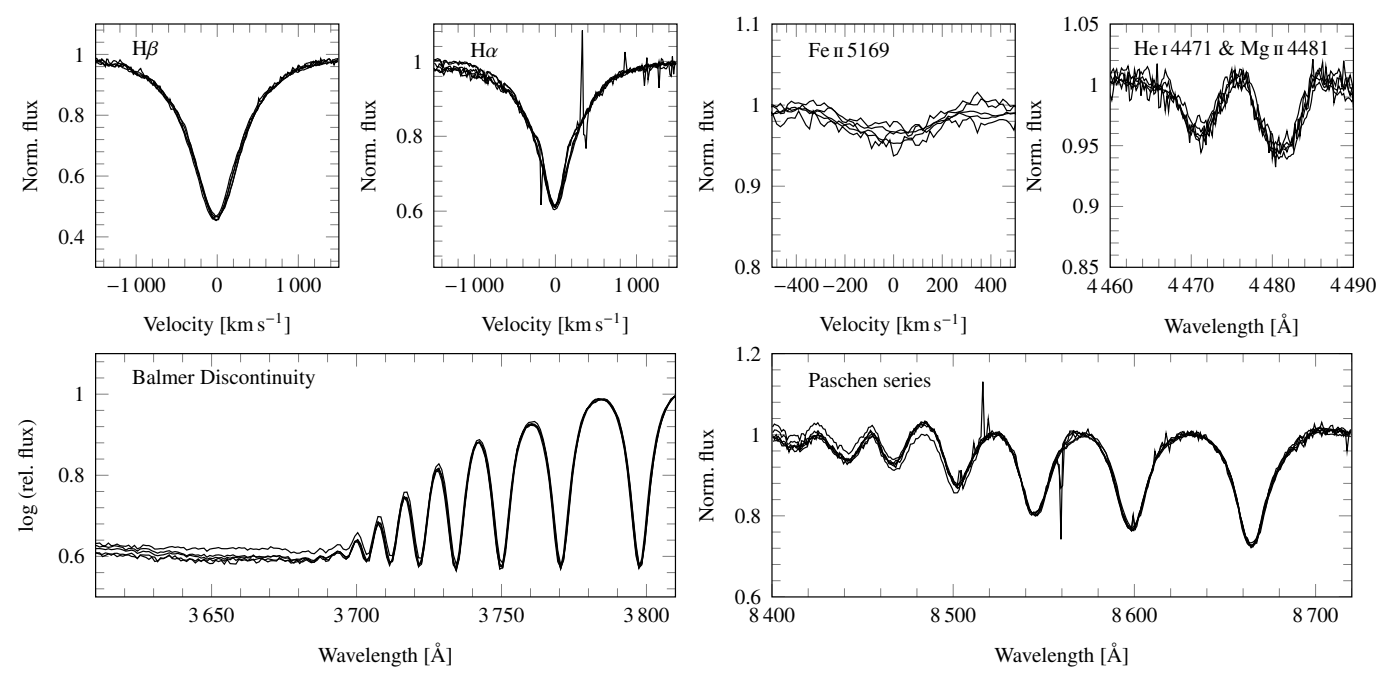

Fig. C.59. Spectrum overview plot for HIP 89500. 

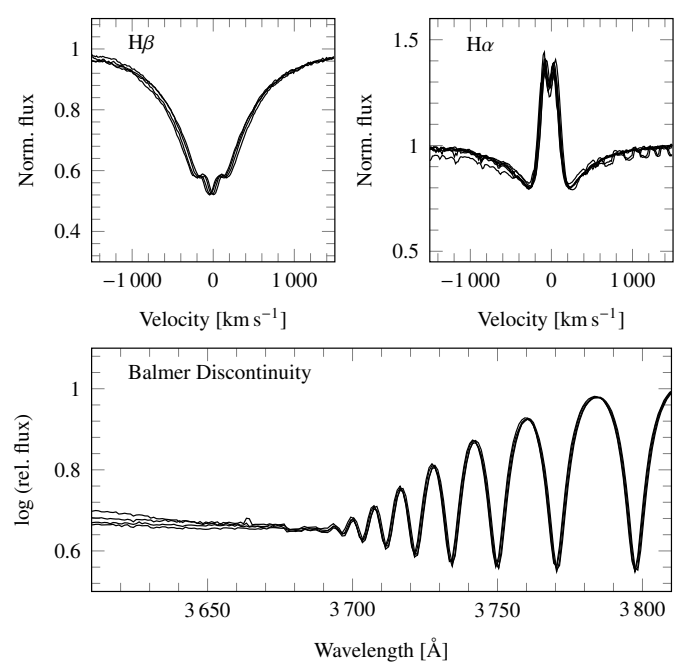

Fig. C.60. Spectrum overview plot for HIP 90096.
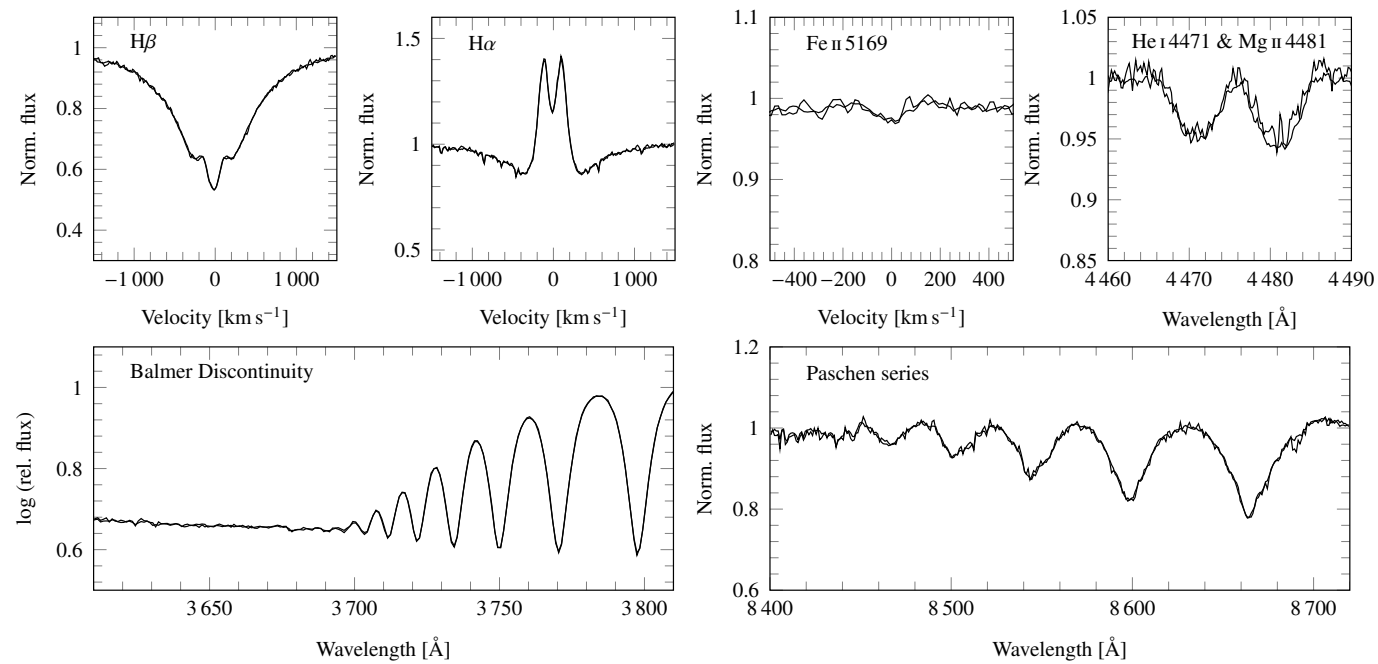

Fig. C.61. Spectrum overview plot for HIP 90509.
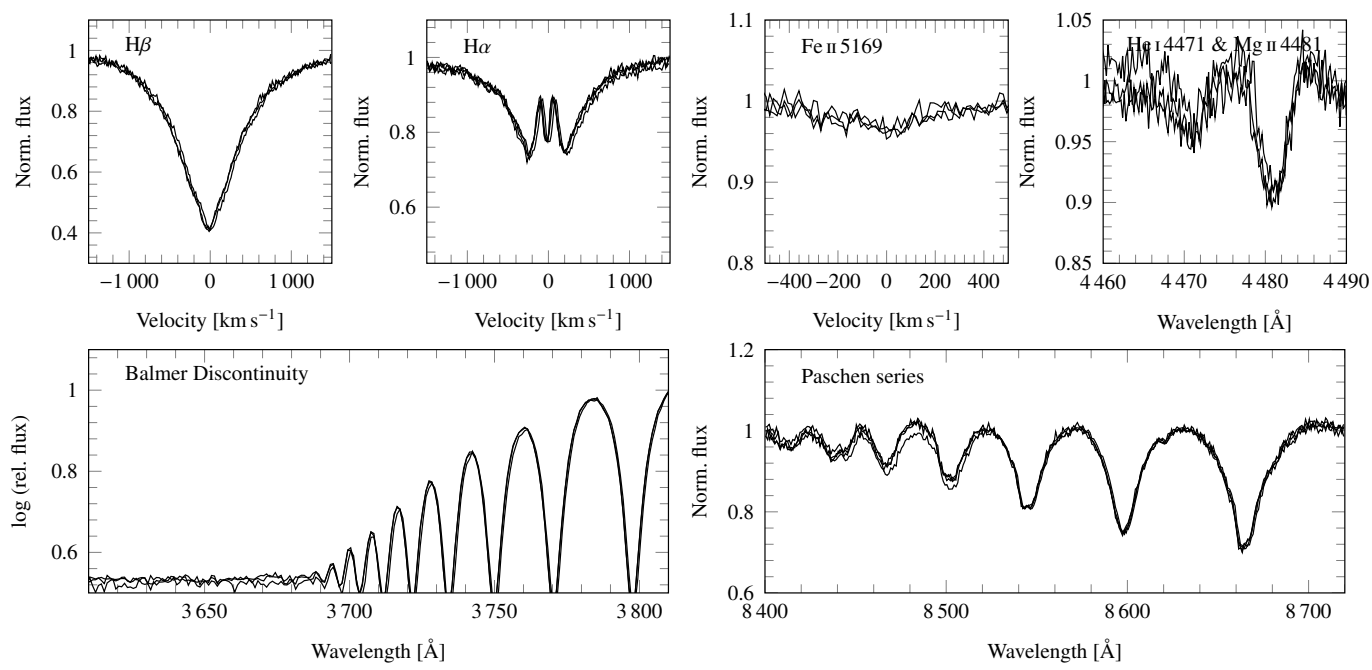

Fig. C.62. Spectrum overview plot for HIP 91460. 
A. Shokry et al.: Stellar parameters of Be stars observed with X-shooter
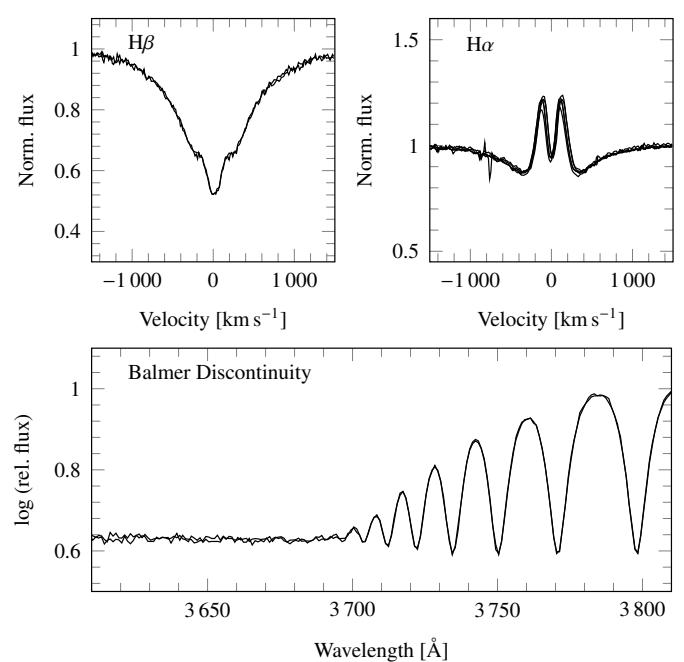

Fig. C.63. Spectrum overview plot for HIP 91975.
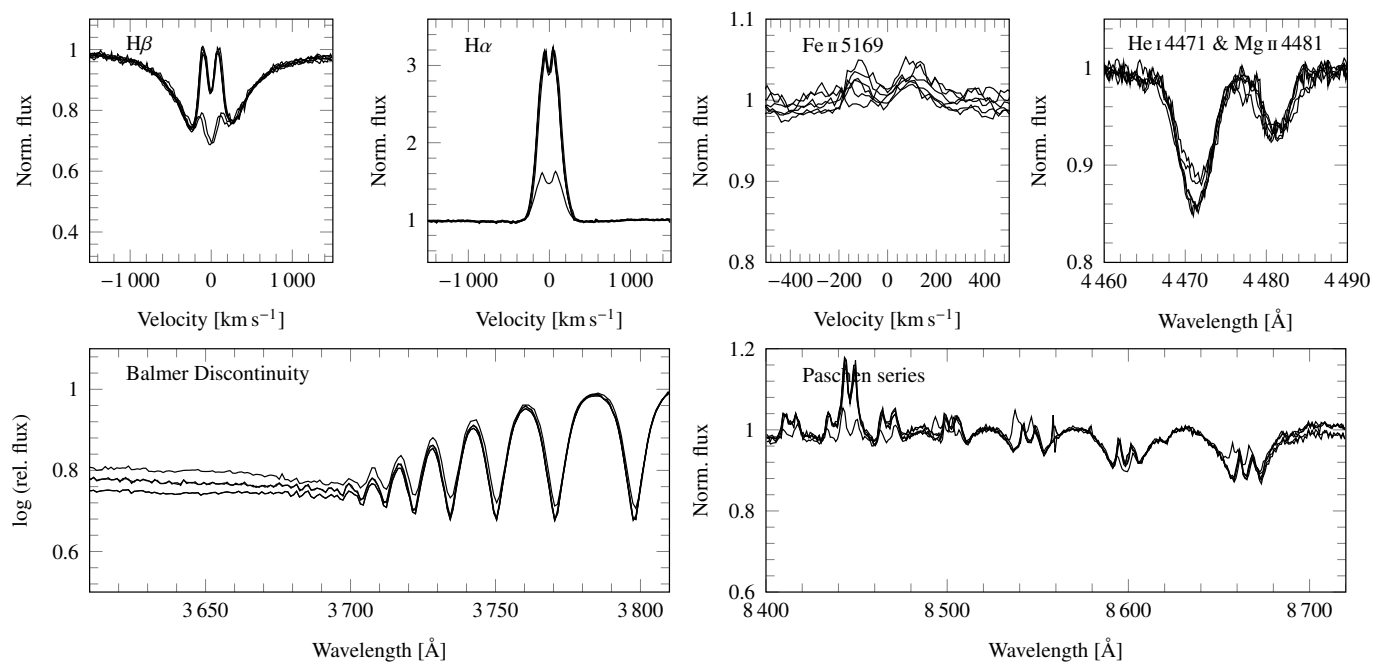

Fig. C.64. Spectrum overview plot for HIP 92038.
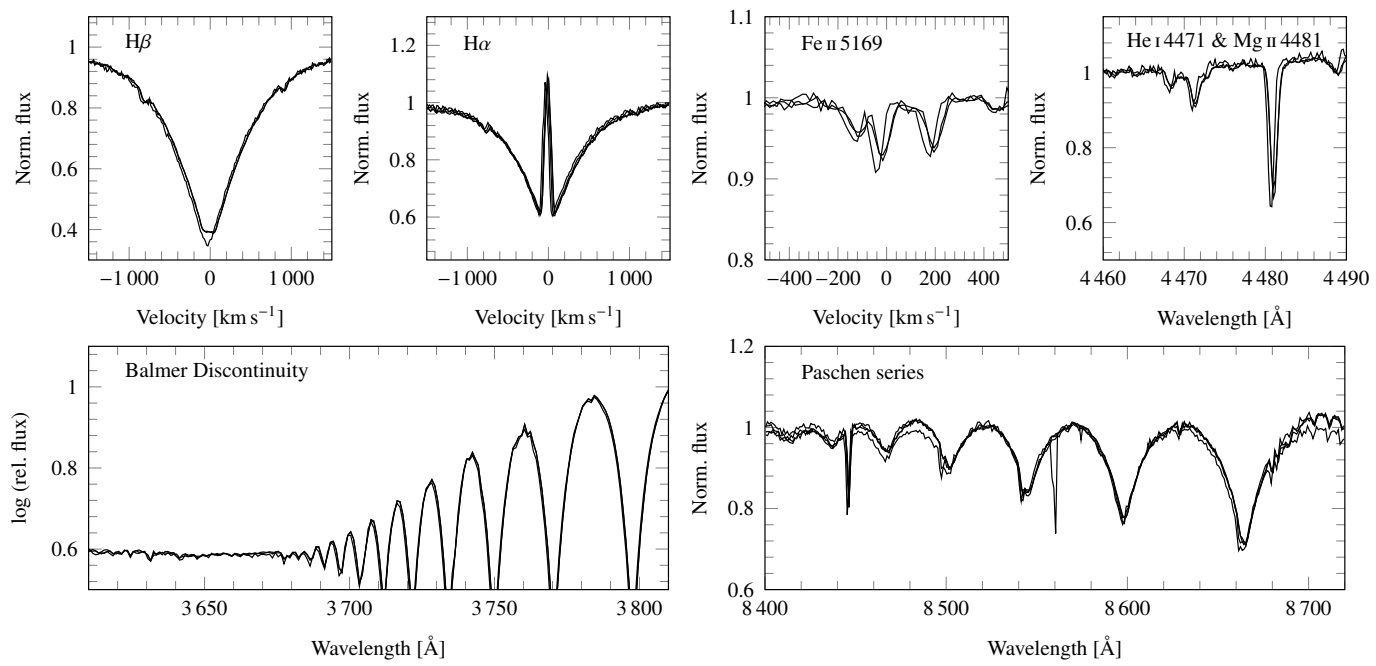

Fig. C.65. Spectrum overview plot for HIP 93993. 

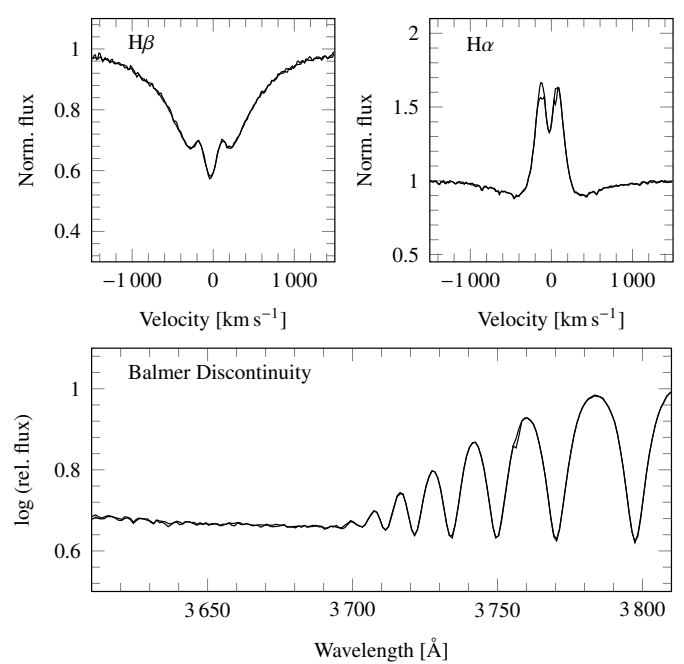

Fig. C.66. Spectrum overview plot for HIP 94770.
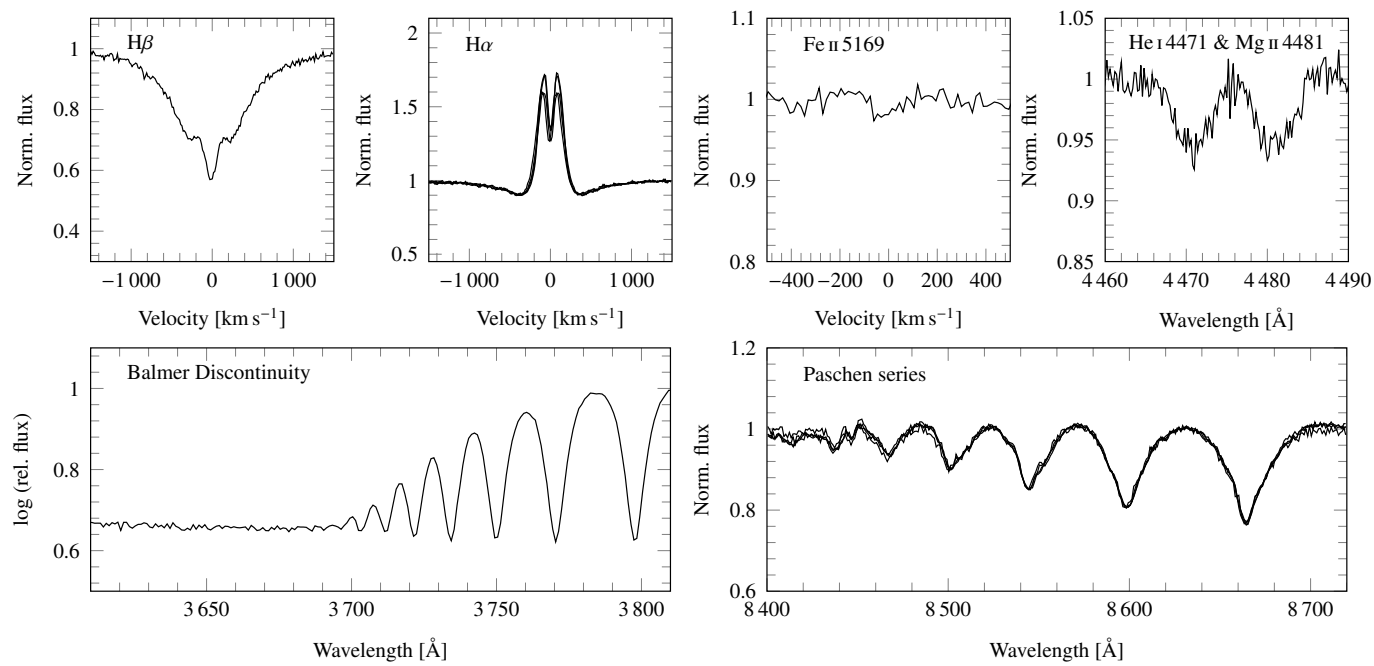
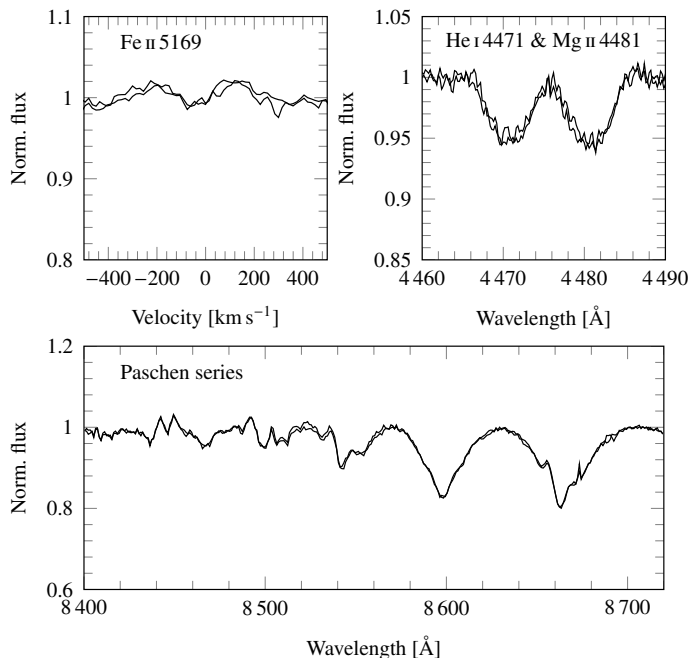

Fig. C.67. Spectrum overview plot for HIP 94859.
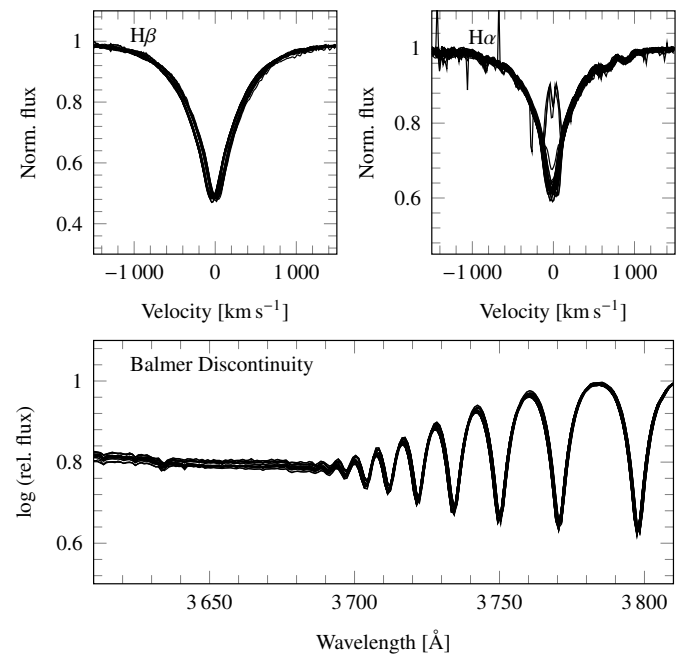
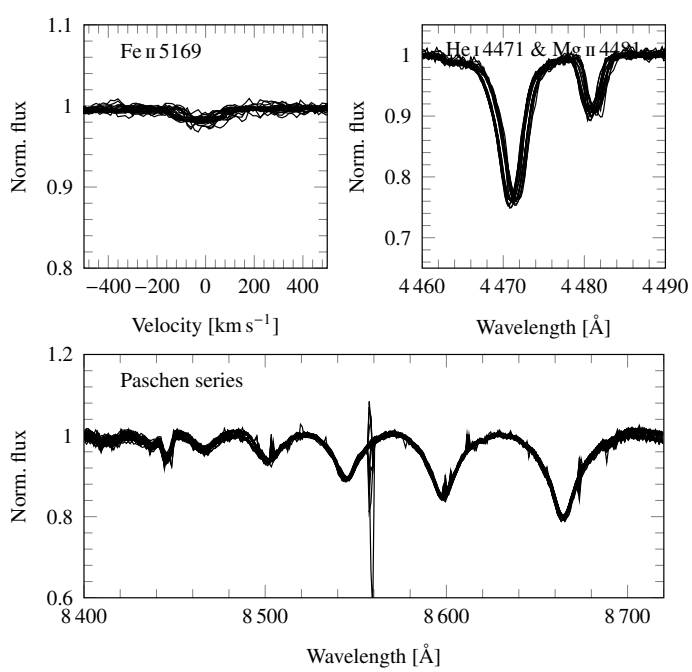

Fig. C.68. Spectrum overview plot for HIP 94986. 
A. Shokry et al.: Stellar parameters of Be stars observed with X-shooter
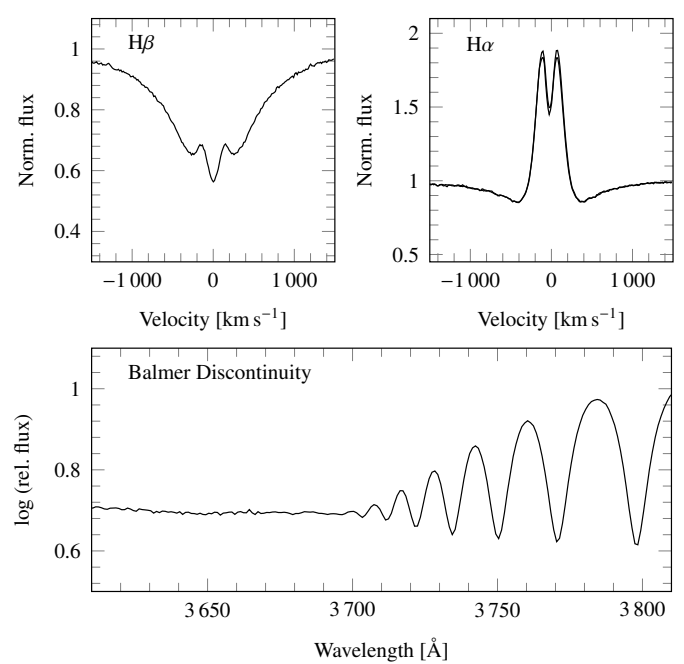

Fig. C.69. Spectrum overview plot for HIP 95109.
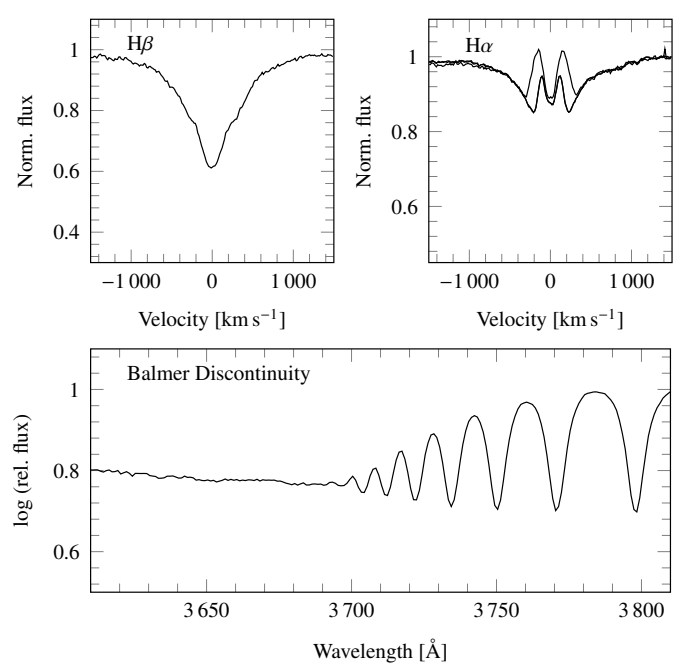

Fig. C.70. Spectrum overview plot for HIP 96453.
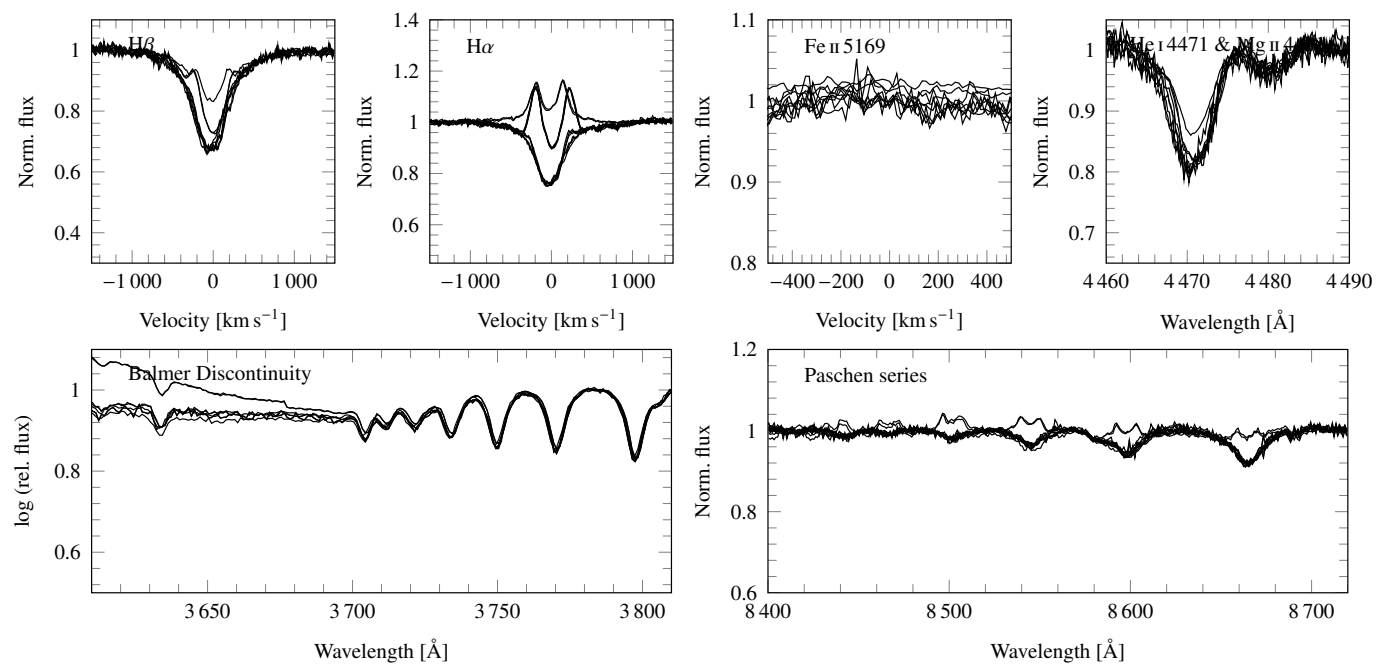

Fig. C.71. Spectrum overview plot for HIP 99457. 

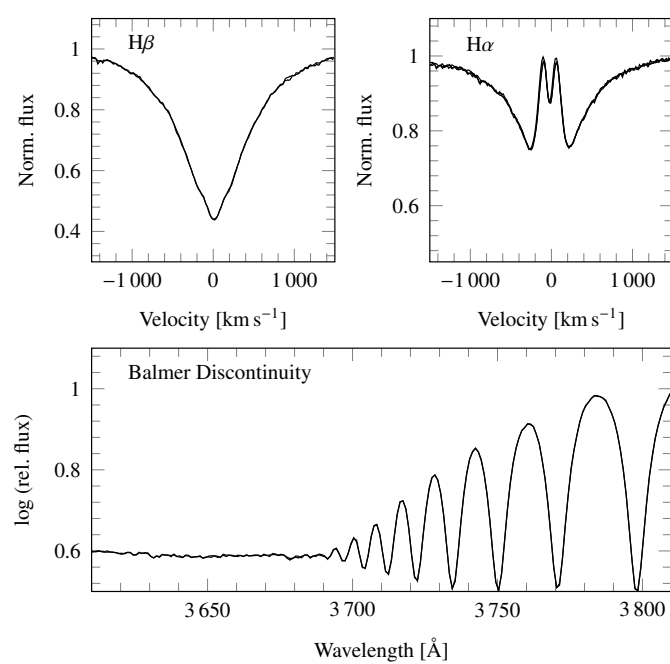

Fig. C.72. Spectrum overview plot for HIP 100664.
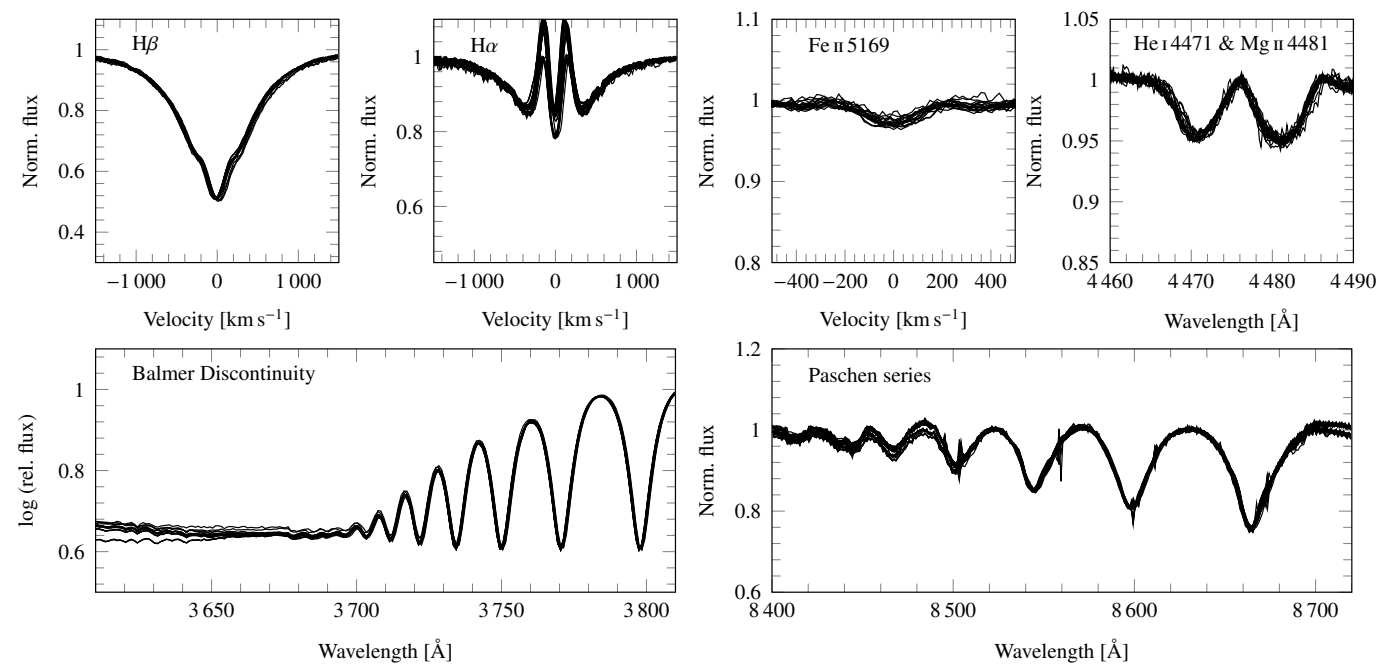

Fig. C.73. Spectrum overview plot for HIP 104508.
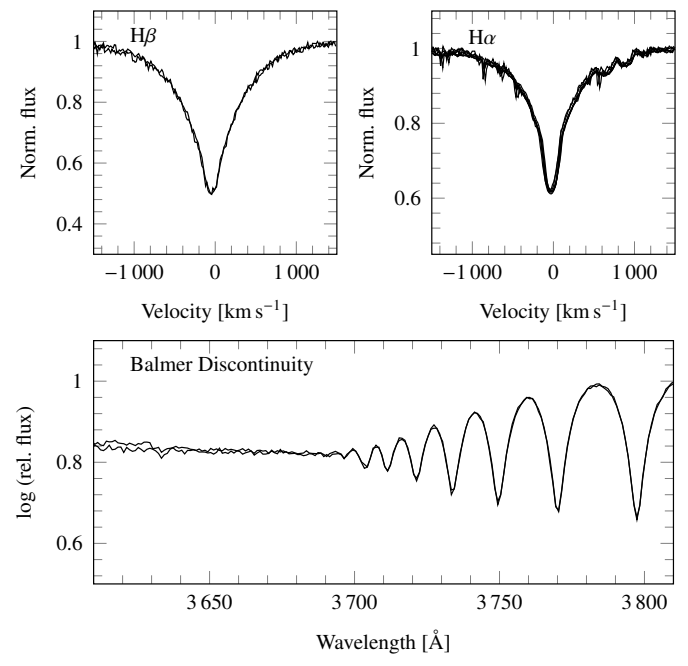
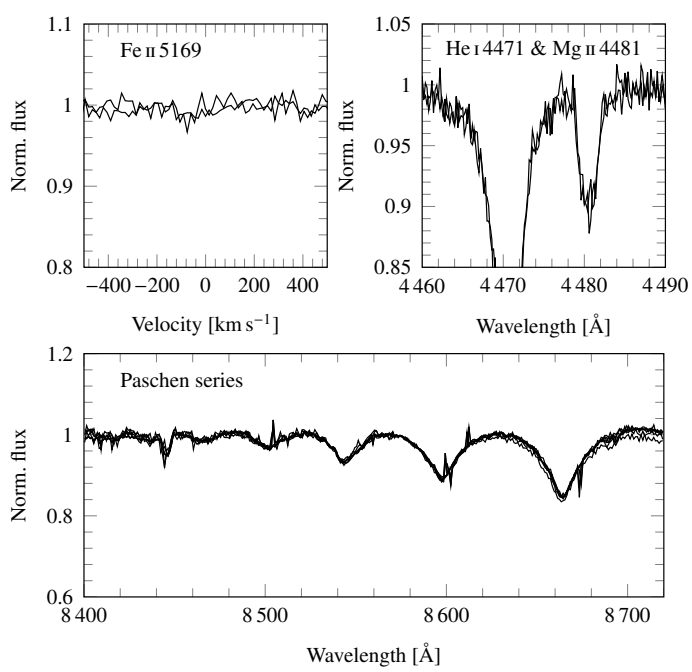

Fig. C.74. Spectrum overview plot for HIP 108022. 
A. Shokry et al.: Stellar parameters of Be stars observed with X-shooter
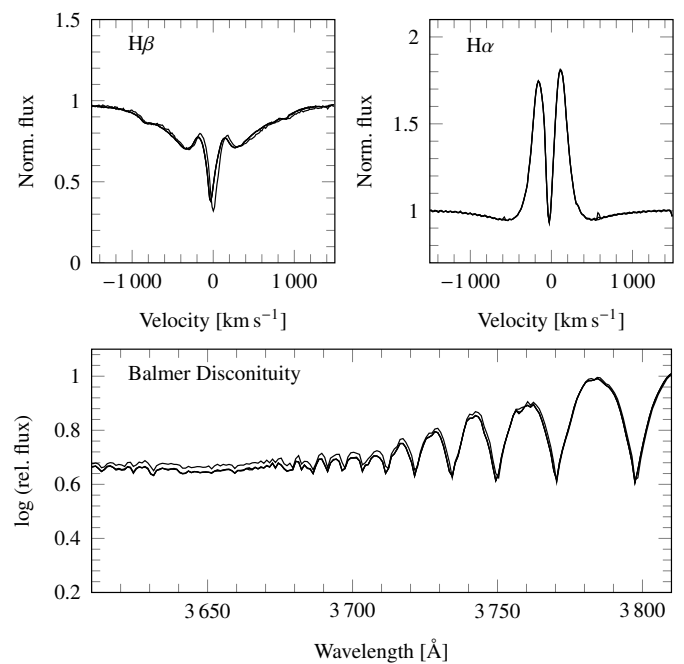

Fig. C.75. Spectrum overview plot for HIP 108402.
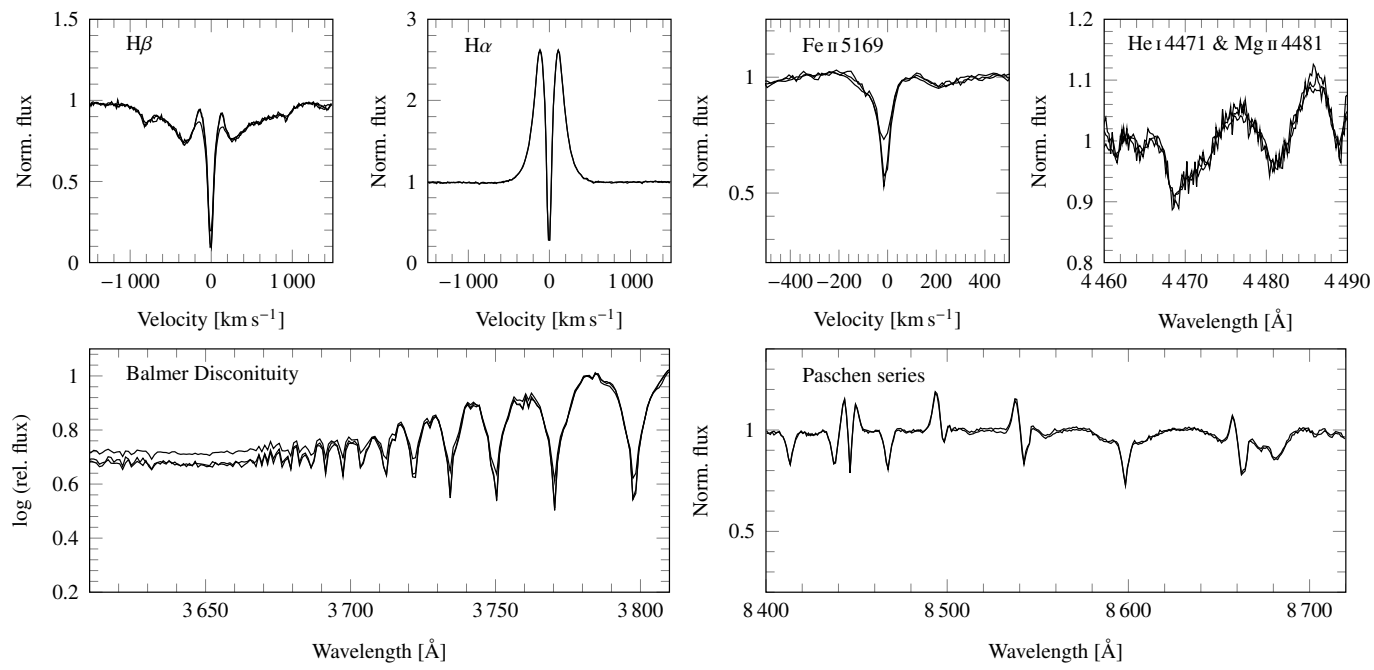

Fig. C.76. Spectrum overview plot for HIP 108597.
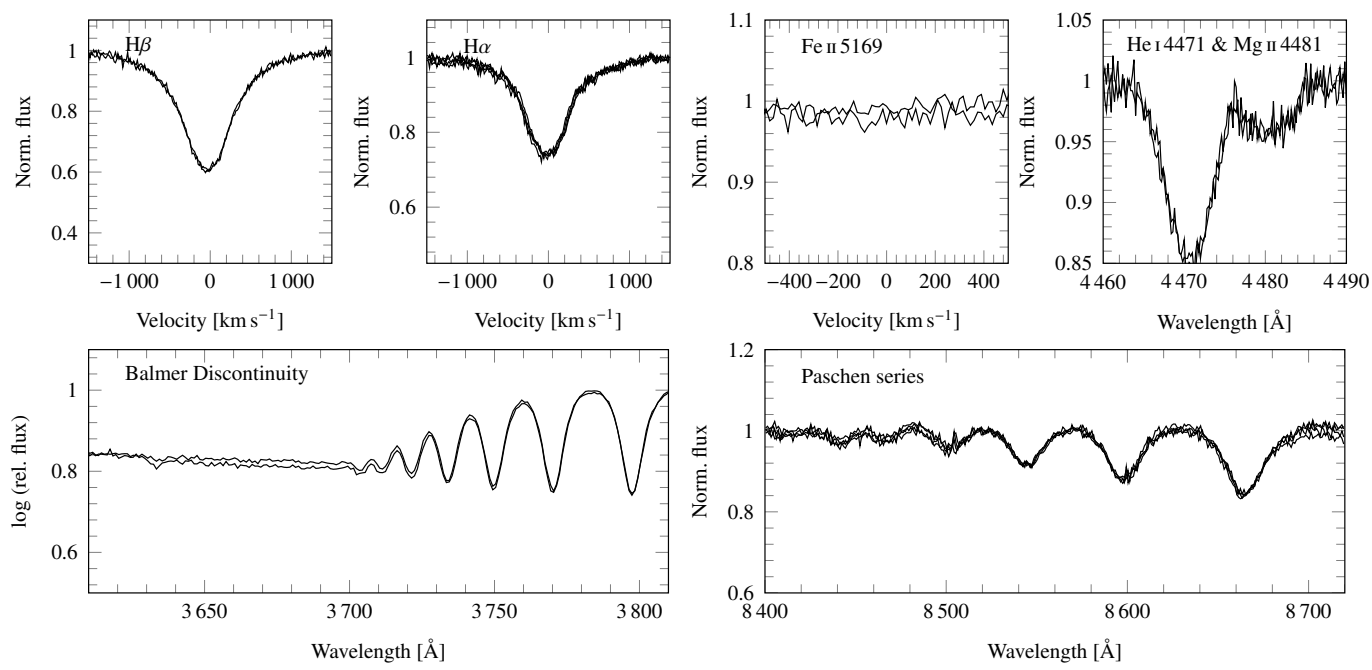

Fig. C.77. Spectrum overview plot for HIP 108975. 